\title{
Physics of high-energy heavy-ion collisions
}

\author{
G Peilert†, H Stöckerł and W Greinerł \\ † Lawrence Livermore National Laboratory, Livermore, CA 94550, USA \\ ‡ Institut für Theoretische Physik, J W Goethe-Universităt, D-60054 Frankfurt am Main, Germany
}

\begin{abstract}
This is a review of the present status of heavy-ion collisions at intermediate energies. The main goal of heavy-ion physics in this energy regime is to shed some light on the nuclear equation of state (EOS), hence we present the basic concept of the EOS in nuclear matter as well as of nuclear shock waves which provide the key mechanism for the compression of nuclear matter.

The main part of this article is devoted to the models currently used for describing heavy-ion reactions theoretically and to the observables useful for extracting information about the EOS from experiments. A detailed discussion of the flow effects with a broad comparison with the available data is presented.

The many-body aspects of such reactions are investigated via the multifragmentation break up of excited nuclear systems and a comparison of model calculations with the most recent multifragmentation experiments is presented.
\end{abstract}

This review was received in June 1993. 


\section{Contents}

1. Introduction

2. The concept of the equation of state and the mechanism of shock compression 536

3. Modelling of heavy-ion reactions 544

3.1. The macroscopic models

3.2. The microscopic models

4. The testing ground for the nuclear EOS

4.1. The collective flow

4,2 . Stopping and thermalization

5. Multifragmentation

6. Conclusion 596

Acknowledgments

References 


\section{Introduction}

Since the beginning of this century the bombarding of heavy nuclei with energetic heavy projectiles has been one of the most important experimental tools for nuclear physicists. The first experiments of this type were performed in the mid $1930 \mathrm{~s}$, using cosmic radiation as projectiles and ionization chambers and Geiger counters as detectors. Later on photographic emulsions were used as a permanently working analogue to the cloud chamber (Schopper 1937, Schopper and Schopper 1939) and a lot of data concerning nucleon-nucleus and nucleus-nucleus collisions up to very high energies have been obtained with cosmic-ray projectiles (Friedländer and Friedmann 1967). Unfortunately cosmic radiation samples a wild mixture of projectiles with different masses, charges and energies. So one had to wait until the mid $1970 \mathrm{~s}$, when several heavy-ion accelerators became available providing the possibility of accelerating heavy ions up to energies of several hundred $\mathrm{MeV}$ per nucleon. The most important facilities are the BEVALAC at Berkeley (which was shut down in early 1993), the GSI at Darmstadt with the UNLAC and the heavy-ion synchrotron SIS, the SATURNE synchrotron at Saclay, the GANL Cyclotron at Caen, the NSCL Superconducting Cyclotron at East Lansing, as well as the facilities in Dubna, Brookhaven, and CERN which provide heavy-ion beams with ultra relativistic energies (for a detailed overview of the experiments see, for example, Kampert (1989), Gutbrod et al (1989a), Schmidt (1991), Schmidt and Schukraft (1993)).

With the invention of these accelerators it became possible to study hot and dense nuclear matter through heavy-ion collisions in a Iaboratory environment, and, indeed, this new field of physics with all its fascinating features was opened for investigation.

A central role in hot and dense nuclear matter physics is played by the nuclear equation of state (EOS) which contains the intriguing possibilities of various phase transitions (liquidgas, meson condensate, quark-gluon plasma, etc) and it is important for the understanding of the physics of the early universe and the static and dynamical behaviour of stars (e.g., supernovae explosions (Müller 1990)). It is therefore one of the main goals of the heavyion experiments to extract the nuclear EOS, i.e. the density dependence of the thermostatic properties of nuclear matter (energy and pressure) from observations. This, however, can only be done if it is possible to compress nuclear matter to high densities and to heat it to high temperatures in the laboratory. The only mechanism known to achieve this is nuclear shock wave compression and heating in high-energy heavy-ion collisions (Scheid et al 1968, 1974, Chapline et al 1973). These early papers can be considered as the starting point of high-energy heavy-ion physics. Scheid et al $(1968,1974)$ discussed, in particular, for the first time the collective flow that until now has been the most prominent signal for nuclear compression. If part of the projectile-target system has been compressed and heated up by such a mechanism, collective flow will appear and the matter will start to expand freely and, in this expansion stage, it will also probe the Eos at low densities. However, all these processes are not directly accessible in an experiment. All that can be measured are the finalstate observables ('the ashes out of the compression zone') that emerge out of the expansion stage. Their final states will probably be highly distorted from interactions taking place after the compression stage. The only possibility for learning something from the experimental observables is therefore to compare them with complete theoretical descriptions of heavyion collisions utilizing different physical scenarios as input. The purpose of this review is to present the most important dynamical models that are used to describe heavy-ion reactions theoretically and to introduce the observables that can be used to extract information about the EOS. Since our main goal is to learn about the EOS, we present in section 2 the basic concept of the EOS of nuclear matter and give a short overview of the basic features of nuclear 
shock waves. In section 3 we present the most important models used to describe heavy-ion collisions. The huge variety of different models can be grouped into two categories. The so-called macroscopic models are presented in section 3.1. They describe the reaction via macroscopic, thermodynamical quantities such as energy, pressure, entropy and density. The equations of motion are the hydrodynamical equations which describe the conservation laws for energy, momentum and matter flow. The macroscopic models have the great advantage that they use the EOS directly as input. This makes these models best suited for a qualitative and even semi-quantitative investigation of the effects of the EOS on the final stage of the reaction. However, the concepts of the macroscopic models rely-at least-on local equilibrium and it is yet not clear whether any form of equilibrium is achieved in heavy-ion reactions at higher energies.

On the other hand, there exist various models that describe heavy-ion reactions microscopically in terms of the dynamics of the interacting nucleons. The most important models of this group, i.e. the Vlasow-Uehling-Uhlenbeck (VUU) model and the quantum molecular dynamics (QMD) model, are presented in section 3.2.

Section 4 deals with the most promising observables that have so far been found to give hints on the EOS. The first observable is the collective flow that had been proposed by early hydrodynamical models to depend strongly on the EOS (Scheid et al 1968, 1974, Baumgardt et al 1975). The in-plane transverse flow as well as the out-of-plane squeeze-out have experimentally been observed. We present detailed investigations of these observables and compare the experimental data with the model results in section 4.1 .

In section 4.2 the stopping power in heavy-ion collisions is investigated. The degree of thermalization is determined microscopically and the extent to which the basic assumptions of the macroscopic models are justified is discussed.

In section 5 we switch over to the physics dealing with the low density part of the EOS. In low-energy heavy-ion collisions the collision path may cross the boundary line between nuclear liquid and vapour which may result in a phase transition with an increase in the total entropy and the fragmentation of the system. While the entropy is not directly accessible the formation of intermediate mass fragments can be observed. Therefore we devote this section to the multifragmentation properties in heavy-ion collisions. This topic has gained much interest recently, since it became, for the first time, possible to study multifragmentation in exclusive experiments. We confront in detail the QMD calculations with the experiments.

The ultra relativistic heavy-ion collisions with $E_{\text {Beam }} / A \gg 2 A \mathrm{GeV}$ are not discussed in this review. This interesting subject, even though smoothly connected to the present topic is beyond the scope of this article and will be presented separately. This procedure is justified by the many new phenomena appearing through the creation of new mesons and baryons and their antiparticles and their mutual annihilation. We also cannot discuss the manifold of the, physically, most exciting signals which deal with the produced particles (e.g. pions, kaons, di-leptons etc); these will also be presented separately.

\section{The concept of the equation of state and the mechanism of shock compression}

Suppose there is infinite nuclear matter, consisting of nucleons only (the inclusion of resonances is then straightforward). If we cut out a piece of volume $V$ and ask for the energy content in that volume, it will be given by

$$
E_{V}=\int_{V} \varrho(r) \cdot W[\varrho(r), T(r)] d V
$$


It is this energy density functional $W(\varrho, T)$ that we call in this simple scenario the equation of state (EOS). It contains all the important thermodynamical features of nuclear matter. For example the pressure is given by (Eisenberg and Greiner 1987)

$$
\begin{aligned}
P(V, T) & =-\left(\frac{\partial E}{\partial V}\right)_{T, N=\text { constant }}=\frac{N}{V^{2}}\left(\frac{\partial E}{\partial \varrho}\right)_{T, N=\text { constant }} \\
& =\frac{N^{2}}{V^{2}}\left(\frac{\partial}{\partial \varrho} \frac{E}{N}\right)_{T, N=\text { constant }}=\varrho^{2}\left(\frac{\partial W}{\partial \varrho}\right)_{T, N=\text { constant }}
\end{aligned}
$$

For $T=0$ the saturation of the nuclear forces leads to a minimum of $W(\varrho, T)$ at the saturation or equilibrium density $Q_{0}$. Fits to nuclear binding energies and density distributions lead to (Scheid et al 1968, 1974, Stock 1975, Blaizot 1980)

$$
\varrho_{0}=0.166 \pm 0.027 \mathrm{fm}^{-3} \quad W\left(\varrho_{0}, T=0\right)=-16 \pm 0.5 \mathrm{MeV} / \text { nucleon }
$$

The curvature of $W(\varrho, T=0)$ near $\varrho_{0}$ characterizes the compressibility of nuclear matter. Larger curvature obviously implies that more energy is necessary for compression. The nuclear (in)compressibility constant $K$ is defined by the change in energy of a nucleus as a reply to a change in the radius.

$$
K=R^{2} \frac{\mathrm{d}^{2} W}{\mathrm{~d} R^{2}}=9\left(\frac{P}{\varrho}+\varrho^{2} \frac{\partial^{2} W}{\partial \varrho^{2}}\right)_{T, N=\text { constant }} .
$$

At $Q=Q_{0}$, the pressure $P$ vanishes per definition and the compressibility constant is given by

$$
K=9 \varrho_{0}^{2}\left(\frac{\partial^{2} W}{\partial \varrho^{2}}\right)_{T, N=\text { constant }} .
$$

The curvature of the EOS can be determined from giant monopole vibrations in spherical nuclei and was measured to (Blaizot 1989, Sharma 1989, Blaizot et al 1976)

$$
K=210 \pm 30 \mathrm{MeV}
$$

but other groups prefer values of $290 \pm 20 \mathrm{MeV}$ (Co and Speth 1986). Clearly there are great uncertainties in measuring even the compressibility constant at normal nuclear matter density. The problem rests in the unknown collective mass $B$ which enters the monopole energy.

It is appropriate, at least in principle, to find a reasonable theory of nuclear matter, that predicts the nuclear matter properties from first principles. This would mean that one has to develop a many-body theory for nuclear matter as a system of interacting nucleons for which the parameters defining the nucleon-nucleon interaction describe both the behaviour of nucleons in the vacuum (i.e. the nucleon-nucleon scattering phase shifts) and the properties of nuclear matter (i.e. saturation density and binding energy). Calculations done so far using realistic nucleon-nucleon interactions which were fitted to describe the free scattering data have failed to describe nuclear matter properties (Green 1976, Anastasio et al 1978, Day and Coester 1976, Lagaris and Pandharipande 1981, Day 1981, Bäckmann et al 1972). Potentials which reproduce the correct saturation density predict a binding energy per nucleon which is too small by roughly $5 \mathrm{MeV}$ and those potentials which predict the correct binding energy 
yield saturation densities twice as large as the empirical value. There is, however, evidence that this problem may be solved by taking into account higher correlations (Dickhoff et al 1982) or relativistic effects (Anastasio et al 1983, Brockmann and Machleidt 1984, Serot and Walecka 1986).

This, however, has not yet been achieved. So the procedure is as follows. First one has to find some reasonable parametrizations of the EOS that can be used as an input for theoretical models, which should then be tested by comparisons with experimental data. The main difficulties here are to find the proper theoretical models for nuclear dynamics and then to find the appropiate experimental observables which are sensitive to the EOS of cold nuclear matter.

Before doing so, we will incorporate the more or less known properties of nuclear matter, i.e. the saturation density $\varrho_{0}$, the binding energy $W_{0}$, the compressibility constant $K$ and the effective mass $m^{*}$ of the nucleons, into a self-consistent phenomenological approach to nuclear matter. Since the last two quantities $\left(K\right.$ and $m^{*}$ ) are known only very imprecisely we here have the opportunity to test how a change in these ground-state properties will influence the high-density part of the EOS. For this purpose we use the self-consistent relativistic meanfield model, developed by Dürr (1956) and later by Walecka (1974) and Serot and Walecka (1985) and further by Boguta and Bodmer (1977) and Boguta and Stöcker (1983).

The relativistic mean-field model discussed here (see Theis et al 1983, Waldhauser et al 1987, 1988) consists of nucleons (and deltas) obeying the Dirac equation, of a classical spin-zero attractive meson field $(\varphi)$ obeying the Klein-Gordan equation, of a spin-one repulsive meson field $\left(V_{\mu}\right)$ obeying the Proca equation and a meson-baryon interaction between them. The scalar interaction includes explicitly nonlinear terms, which allow a more realistic description of the nuclear matter properties.

The model Lagrangian is

$$
\begin{gathered}
L=\bar{\Psi}_{\mathrm{N}}\left(\mathrm{i} \gamma_{\mu} \partial^{\mu}-m_{N}\right) \Psi_{N}-U(\varphi)-\frac{1}{2} \partial_{\mu} \varphi \partial^{\mu} \varphi-\frac{1}{4} F_{\mu \nu} F^{\mu \nu}-\frac{1}{2} m_{\nu}^{2} V_{\mu} V^{\mu} \\
-g_{\mathrm{s}}^{N} \bar{\Psi}_{N} \Psi_{N} \varphi-g_{\mathrm{v}}^{N} \bar{\Psi}_{\mathrm{N}} \gamma_{\mu} \Psi_{N} V^{\mu}
\end{gathered}
$$

where the field tensor is defined as

$$
F_{\mu \nu}=\frac{\partial V_{\nu}}{\partial x_{\mu}}-\frac{\partial V_{\mu}}{\partial x_{\nu}} .
$$

The potential function $U(\varphi)$ is taken as

$$
U(\varphi)=\frac{1}{2} m_{s}^{2} \varphi^{2}+\frac{1}{3} b \varphi^{3}+\frac{1}{4} c \varphi^{4} .
$$

For rotationally and translationary invariant symmetric nuclear matter the field equations for the meson field, in the mean-field approximation, are

$$
\begin{aligned}
& m_{\mathrm{s}}^{2} \varphi_{0}+b \varphi_{0}^{2}+c \varphi_{0}^{3}=-g_{\mathrm{s}}^{N} \varrho_{\mathrm{s}}^{N} \\
& m_{\mathrm{v}}^{2} V_{0}=g_{\mathrm{v}}^{N} \varrho_{\mathrm{v}}^{N} \\
& V=0 .
\end{aligned}
$$

The energy density is given by

$$
\varepsilon=\frac{1}{2} \frac{\left(g_{\mathrm{v}}^{N}\right)^{2}}{m_{\mathrm{v}}^{2}} \varrho_{\mathrm{B}}^{2}+U\left(\varphi_{0}\right)+\frac{\gamma_{\mathrm{N}}}{(2 \pi)^{3}} \int_{0}^{\infty} \mathrm{d}^{3} k\left(k^{2}+m_{\mathrm{N}}^{* 2}\right)^{1 / 2}[n(T)+\bar{n}(T)]
$$


where the effective mass $m_{\mathrm{N}}^{*}$ of the nucleon is defined as $m_{\mathrm{N}}^{\star}=m_{\mathrm{N}}+g_{\mathrm{s}}^{N} \varphi_{0}$. The degeneracy factor $\gamma_{\mathrm{N}}=4$ corresponds to spin-isospin $1 / 2$ nucleons. The four relevant parameters of the model, the dimensionless coupling constants

$$
C_{s}=\frac{g_{\mathrm{s}}^{N} m_{\mathrm{N}}}{m_{\mathrm{s}}} \quad C_{v}=\frac{g_{\mathrm{v}}^{N} m_{\mathrm{N}}}{m_{\mathrm{v}}} \quad B=\frac{b}{\left(g_{\mathrm{s}}^{N}\right)^{3} m_{\mathrm{N}}} \quad C=\frac{c}{\left(g_{\mathrm{s}}^{N}\right)^{4}}
$$

are adjusted to fit the ground-state properties for cold $(T=0)$ nuclear matter (for details of the parametrization see Waldhauser et al (1988)).

Now we can study the dependence of the EOS on the ground-state properties $m_{\mathrm{N}}^{\star}\left(\varrho_{0}\right)$ and $K$. Figure 1 shows the strong dependence of the EOS on the effective mass, when a fixed compressibility constant is used, while figure 2 shows the influence of the compressibility constant $K$ for a fixed value of the effective mass. These results reveal the difficulties one encounters if one wants to extract the EOS from heavy-ion data, where one will always be dealing with compressed and hot nuclear matter, permanently changing due to the violent dynamics.

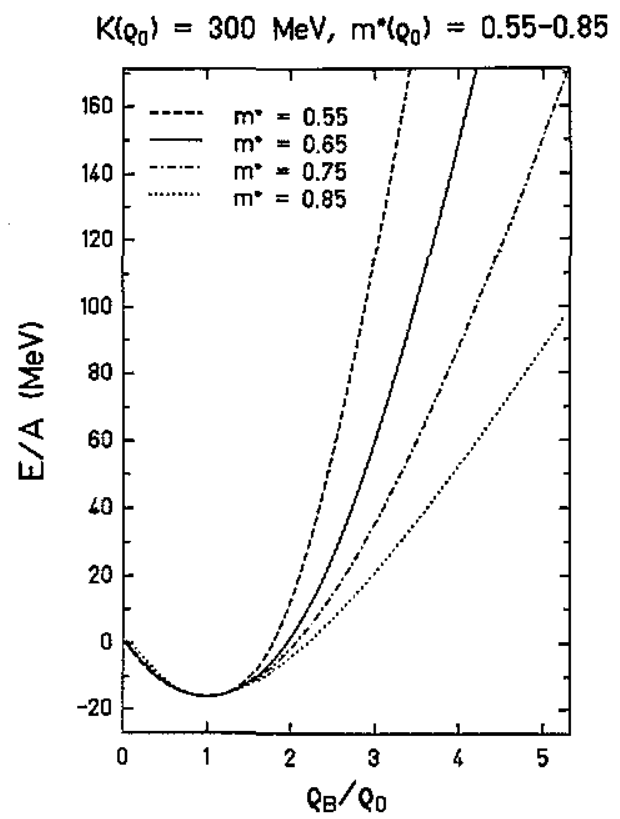

Figure 1. The Eas for cold nuclear matter is shown for fixed ground-state binding energy, baryon density and ground-state compressibility for different effective masses as indicated (Waldhauser et al 1988).

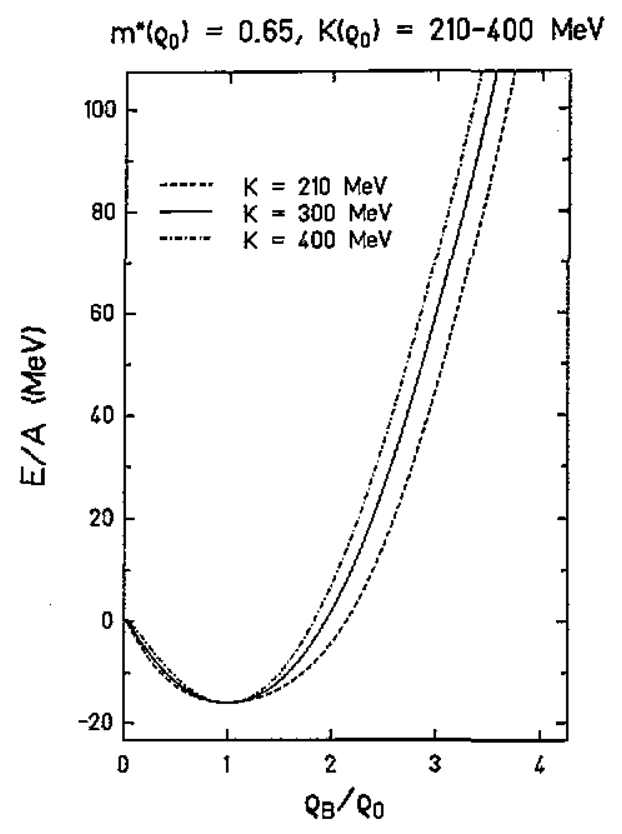

Figure 2. The Eos for a fixed value of the ground-state effective mass and various values of the ground-state compressibility (Waldhauser et al 1988).

Next we investigate possible phase transitions that are favoured by such an EOs. Therefore we additionally include a delta field $\Psi_{\Delta}$ in our approach and end up with the following Langrangian density:

$$
\begin{aligned}
L=\bar{\Psi}_{\mathrm{N}}\left(\mathrm{i} \gamma_{\mu} \partial^{\mu}\right. & \left.-m_{\mathrm{N}}\right) \Psi_{\mathrm{N}}+\bar{\Psi}_{\Delta}\left(\mathrm{i} \gamma_{\mu} \partial^{\mu}-m_{\Delta}\right) \Psi_{\Delta} \\
& -\frac{1}{2} \partial_{\mu} \varphi \partial^{\mu} \varphi-U(\varphi)-\frac{1}{4} F_{\mu \nu} F^{\mu \nu}-\frac{1}{2} m_{\mathrm{v}}^{2} V_{\mu} V^{\mu} \\
& -g_{\mathrm{s}}^{N} \bar{\Psi}_{\mathrm{N}} \Psi_{\mathrm{N}} \varphi-g_{\mathrm{s}}^{\Delta} \bar{\Psi}_{\Delta} \Psi_{\Delta} \varphi-g_{\mathrm{v}}^{N} \bar{\Psi}_{\mathrm{N}} \gamma_{\mu} \Psi_{\mathrm{N}} V^{\mu}-g_{\mathrm{v}}^{\Delta} \bar{\Psi}_{\Delta} \gamma_{\mu} \Psi_{\Delta} V^{\mu}
\end{aligned}
$$


The field equations for the nucleon, delta, scalar and vector fields are now given by

$$
\begin{aligned}
& \left\{\mathrm{i} \gamma_{\mu} \partial^{\mu}-\left[m_{\mathrm{N}}+g_{\mathrm{s}}^{N} \varphi_{0}\right]-g_{\mathrm{v}}^{N} \gamma_{0} V^{0}\right\} \Psi_{\mathrm{N}}=0 \\
& \left\{\mathrm{i} \gamma_{\mu} \partial^{\mu}-\left[m_{\Delta}+g_{\mathrm{s}}^{\Delta} \varphi_{0}\right]-g_{\mathrm{v}}^{\Delta} \gamma_{0} V^{0}\right\} \Psi_{\Delta}=0 \\
& m_{\mathrm{s}}^{2} \varphi_{0}+b \varphi_{0}^{2}+c \varphi_{0}^{3}=-g_{\mathrm{s}}^{N} \varrho_{\mathrm{s}}^{N}-g_{\mathrm{s}}^{\Delta} \varrho_{\mathrm{s}}^{\Delta} \\
& m_{\mathrm{v}}^{2} V_{0}=g_{\mathrm{v}}^{N} \varrho_{\mathrm{v}}^{N}+g_{\mathrm{v}}^{\Delta} \varrho_{\mathrm{v}}^{\Delta} .
\end{aligned}
$$

The effective masses of the nucleons and the deltas are given by $m_{\mathrm{N}}^{*}=m_{\mathrm{N}}+g_{\mathrm{s}}^{\mathrm{N}} \varphi_{0}$, $m_{\Delta}^{\star}=m_{\Delta}+g_{\mathrm{s}}^{\Delta} \varphi_{0}$, respectively. The two additional parameters are the delta coupling constants

$$
\alpha=g_{\mathrm{v}}^{\Delta} / g_{\mathrm{v}}^{N} \quad \text { and } \quad \beta=g_{\mathrm{s}}^{\Delta} / g_{\mathrm{s}}^{N}
$$

describing the coupling to the vector and scalar meson fields, respectively.

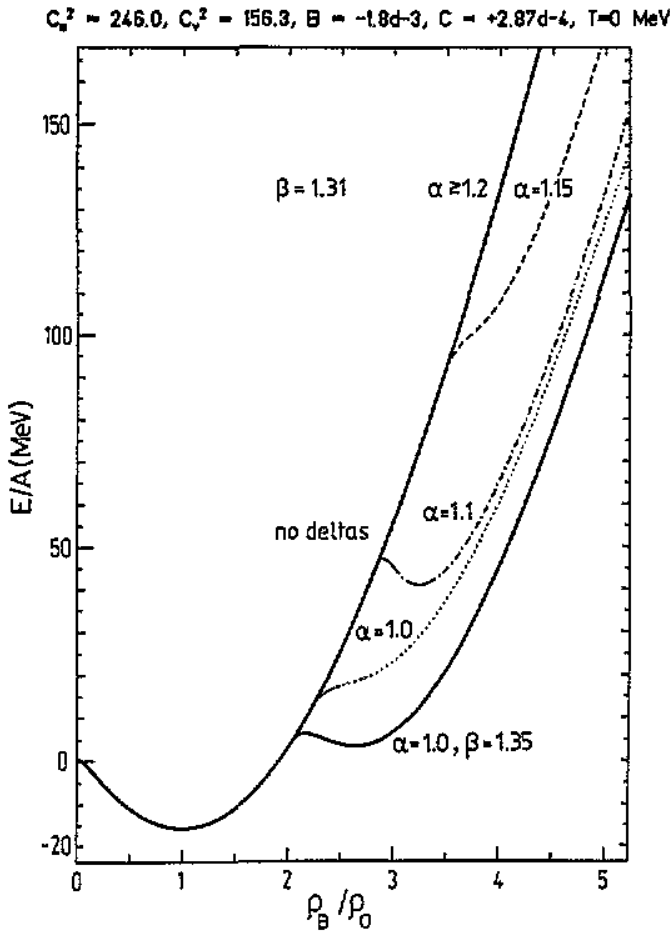

Figure 3. The EOS including delta resonances at $T=$ $0 \mathrm{MeV}$ for different values of the coupling constants $\alpha$ and $\beta$, as indicated (Waldhauser et al 1987).

Let us now study the possible phase structure of nuclear matter. Figure 3 shows the EOS at $T=0 \mathrm{MeV}$ for different values of the scalar and vector coupling constants $\alpha$ and $\beta$. One observes a secondary minimum at densities $\varrho>2 \varrho_{0}$. The reason for this is the rapid increase in the delta production rate at $2 \varrho_{0}$. This reflects the strong attraction of the deltas by the scalar field, which results in a lowering of the delta continuum states below the Fermi surface of the nucleons. Since the delta degeneracy $\left(\gamma_{\Delta}=16\right)$ is much larger than the nucleon degeneracy $\left(\gamma_{N}=4\right)$, the equilibrium is dominated by an $80 \%$ abundance of deltas.

The vector coupling constant $\alpha$ also has a great influence on the EOS as can be seen in figure 3. If $\beta$ is fixed to $\beta=1.31$ and only $\alpha$ is varied, the minimum shifts to higher 


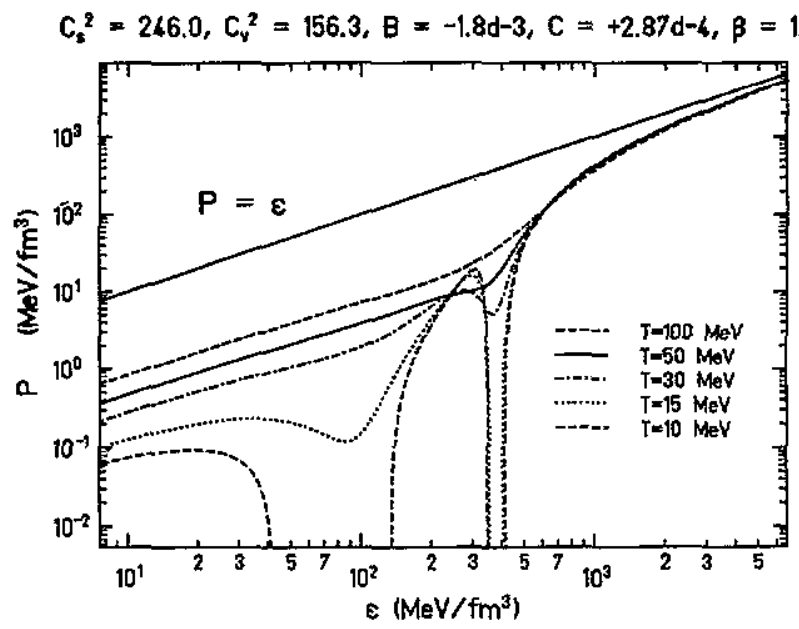

Figure 4. Pressure $P$ against the energy density $\epsilon$ for different temperatures (Waldhauser et al 1987).

densities and vanishes completely for $\alpha>1.15$. Hence we conclude that, in addition to the ground-state properties, the delta coupling constants also have a large influence on the high density behaviour of the EOS.

In figure 4 the isotherms of the pressure against the energy density are shown. In such a diagram the possible phase transitions can be identified very easily. The isotherms separate clearly into four regions, which can be identified with the nucleon-liquid, the nucleon gas, a delta phase and a fourth phase which corresponds to a nearly massless phase of hadrons (Theis et al 1983, Garpman et al 1979, Nakai and Takagi 1984, Glendenning 1984). The massless phase in the meson-hadron field theory may be another description of what is called the quark-gluon plasma in the quark-gluon picture of nuclear matter. Indeed, Rischke et al $(1992 \mathrm{a}, \mathrm{b})$ have recently found in an analysis of lattice gauge calculations of the gluon plasma that strong gluon clusters exist even beyond the phase transition point. One is clearly dealing with a cluster plasma. This is the physical meaning of the phase transition to massless constituents observed here. The Van-der-Waals form of the EOS in the lowtemperature regime is due to the long-range attractive and short-range repulsive forces. We will come back to this point in section 5 . Finally we show in figure 5 the phase diagram of baryonic matter calculated in the relativistic mean-field approach (Waldhauser et al 1987). One recognizes the liquid-gas coexistence, the delta matter and the narrow (in $T$ ) but broad (in $\varrho$ ) band of the transition region to the massless plasma. The liquid-vapour phase transition is not affected by the inclusion of delta resonances while the high-temperature phase transition is strongly shifted to lower temperatures.

As a final point in this section we introduce a set of phenomenological EOss which are used later in the macroscopic and microscopic models of heavy-ion collisions (see sections 3.1 and 3.2 ).

In hydrodynamical calculations one uses the so called linear and quadratic Eos with the parametrizations (Scheid et al 1968, 1974):

$$
\begin{array}{ll}
W(\varrho, T=0)=E_{\text {kin }}+\frac{K}{18 \varrho_{0}^{2}}\left(\varrho-\varrho_{0}\right)^{2}+W_{0} & \text { quadratic EOS } \\
W(\varrho, T=0)=E_{\text {kin }}+\left\{\begin{array}{ll}
\frac{K}{18 \varrho \varrho_{0}}\left(\varrho-\varrho_{0}\right)^{2}+W_{0} & \varrho>\varrho^{\prime} \\
\alpha \varrho & \varrho<\varrho^{\prime}
\end{array}\right. \text { linear EOS }
\end{array}
$$




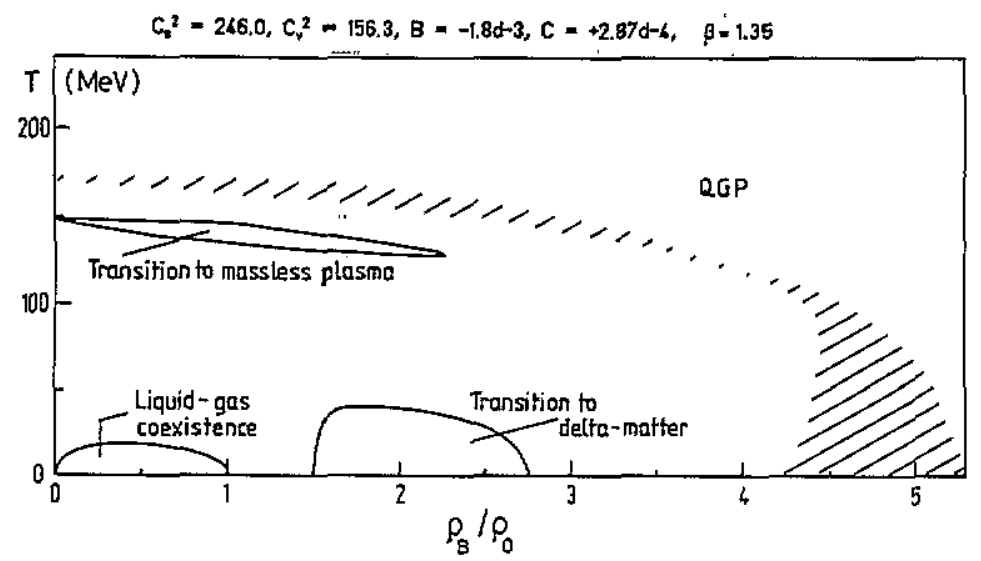

Figure 5. The phase diagram for nuclear matter. The coexistence region for liquid-gas and the transition regions to delta matter and to the massless plasma are displayed. The hatched area indicates the coexistence region of the quark-gluon plasma and hadronic phase (Waldhauser et al 1987).

with $Q^{\prime}=\varrho_{0} /\left(1-9 W_{0} / K\right), \alpha=\left(K / 18 \varrho_{0}\right)\left(1-\varrho_{0}^{2} / \varrho^{2}\right)$.

In the microscopic models a parametrization of the EOS is used which stems originally from Skyrme (1959) and was later on used in Hartree-Fock calculations (Vautherin and Brink 1972). In its most simplified version this EOS is given by (see also section 3.2 .1 and equations (22) ff.)

$$
W(\varrho, T=0)=E_{\text {kin }}+\alpha \varrho+\beta \varrho^{\gamma} .
$$

The parameters $\alpha, \beta$ and $\gamma$ have to be adjusted so as to reproduce the groundstate properties of nuclear matter, i.e. the binding energy, saturation density and the compressibility. The compressibility constants $K$ that are used later on in the vU and QMD models are $380 \mathrm{MeV}$ for a hard $(\mathrm{H})$ EOS and $200 \mathrm{MeV}$ for the soft $(\mathrm{S})$ EOS. Figure 6 shows these four EOSs for different compressibilities as indicated. In all cases the kinetic energy is taken as the energy of an ideal Fermi gas.

\section{Compressional part of the EOS.}

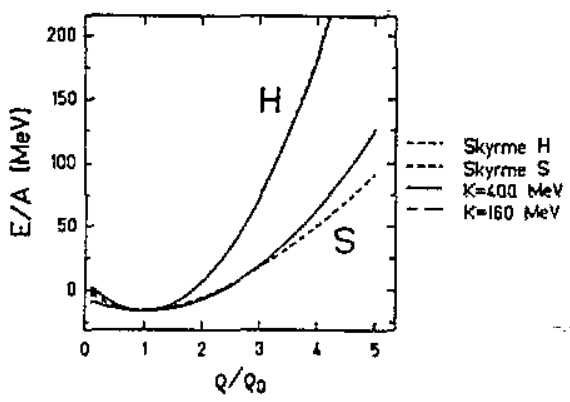

Figure 6. Comparison of the different parametrizations of the eos used in most dynamical models of heavy-ion collisions. The Skyrme EOS (equation (15)) is compared with the quadratic EOS (equation (14)) for two different values of $K$.

The basic question is how can one study the nuclear Eos under extreme conditions, far away from the ground state. The only way to obtain access to the nuclear EOS at densities exceeding the equilibrium density and at high temperatures in the laboratory is through 
relativistic heavy-ion collisions. The possibility of performing heavy-ion collisions under controlled conditions and by varying a series of parameters like the bombarding energy, the impact parameter and the mass of the colliding nuclei should enable us to extract detailed information on the EOS from such experiments. Of course, the internal structure of neutron stars and supernovae is also dependent on the EOS, but from such astrophysical observables only confirmation and compatibility of EOS-models can be expected to be confirmed. The basic mechanism for producing high density matter in relativistic heavy-ion collisions has been proposed by Scheid et al as early as 1968 and extended in 1974 (Scheid et al 1968, 1974). When the relative velocity of two colliding nuclei exceeds the velocity of sound $\left(v_{\text {sound }}=\sqrt{K /\left(9 m_{\mathrm{N}}\right)}\right)$ they predicted the occurrence of shock waves in heavy-ion collisions. To demonstrate the basic physics of compression effects in this case the following very simplified model was used. Assume two identical nuclei the volume of which is divided into three parts (see figure 7), namely an ellipsoid, sandwiched between two cut-off spheres. The nuclear matter is assumed to be homogeneously distributed over the different volumes. Compression is assumed to occur only in the ellipsoidal region. The time-dependent density and pressure in the ellipsoidal region is then determined by the conservation of energy, momentum and matter during the course of the collision.

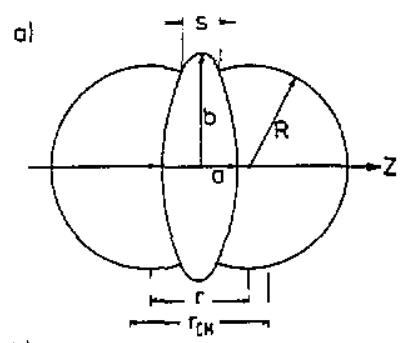

bl

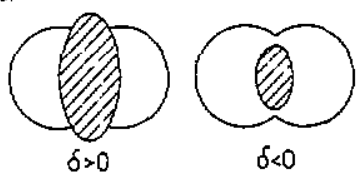

Figure 7. Geometric parameters of the model used in Scheid et al (1974).

The conservation laws for the matter density $\varrho$, the momentum density $M$, pressure $p$ and the energy density $e$ are given by the hydrodynamical equations

$$
\begin{aligned}
& \frac{\partial e_{\mathrm{L}}}{\partial t}+\nabla\left(e_{\mathrm{L}} \frac{v}{c}\right)=-\nabla\left(p \frac{v}{c}\right) \\
& \frac{\partial M^{k}}{\partial t}+\nabla\left(M^{k} \frac{v}{c}\right)=-\nabla^{k} p \quad k=1,2,3 \\
& \frac{\partial \varrho_{\mathrm{L}}}{\partial t}+\nabla\left(\varrho_{\mathrm{L}} \frac{v}{c}\right)=0
\end{aligned}
$$

with

$$
\begin{aligned}
& u^{\mu}=(\gamma, \gamma v / c) \\
& j^{\mu}=\varrho u^{\mu} \\
& T^{\mu \nu}=(e+p) u^{\mu} u^{\nu}-p g^{\mu \nu}
\end{aligned}
$$




$$
\begin{aligned}
& e_{\mathrm{L}}=T^{00}=\gamma^{2}(e+p)-p \\
& Q_{\mathrm{L}}=j^{0}=\gamma \varrho \\
& M^{k}=T^{k 0}=\gamma^{2}(e+p) v^{k} / c .
\end{aligned}
$$

The index ' $\mathrm{L}$ ' stands for local and implies that the corresponding quantities are taken with respect to the local rest frame. The nuclear $\operatorname{EOS} W(\varrho)$ enters directly into these equations through a relationship between the local pressure $p$ and energy density $e$ and the local temperature $T$ and baryon density $\varrho$

$$
e=e(\varrho, T) \quad p=p(\varrho, T) .
$$

In the simple scenario of figure 7 the non-trivial consequences of equations (16) are related to the conservation of energy, momentum and matter across the discontinuous shock front separating the ellipsoidal and the cut-off regions. Matter flowing from the cut-off spheres into the ellipsoidal region will cause the shock front to move outwards. The conditions of continuity of $u^{\mu}$ and $T^{\mu \nu}$ at the shock front leads to the relativistic RankineHugionot equation

$$
\frac{\left(e_{1}+p_{1}\right)^{2}}{\varrho_{1}^{2}}-\frac{\left(e_{0}+p_{0}\right)^{2}}{\varrho_{0}^{2}}=\left(p_{1}-p_{0}\right)\left(\frac{e_{1}+p_{1}}{\varrho_{1}^{2}}+\frac{e_{0}+p_{0}}{\varrho_{0}^{2}}\right)
$$

which can easily be solved (the indices 0 and 1 correspond to the values of $p$ and $e$ on both sides of the shock front). The velocities relative to the shock front are

$$
\left(\frac{v_{\mathrm{s}}}{c}\right)^{2}=\frac{\left(p_{1}-p_{0}\right)\left(e_{1}+p_{0}\right)}{\left(e_{1}-e_{0}\right)\left(p_{1}+e_{0}\right)} \quad\left(\frac{v_{\mathrm{f}}}{c}\right)^{2}=\frac{\left(p_{1}-p_{0}\right)\left(e_{1}+e_{0}\right)}{\left(e_{1}+p_{0}\right)\left(p_{1}+e_{0}\right)} .
$$

Both the shock-front velocity $v_{s}$ and the flow velocity $v_{\mathrm{f}}$ are functions of $\varrho$ and $T$; for a large density $\varrho$ in the shock front these velocities approach the velocity of light. Figure 8 depicts the dependence of $\varrho$ on the shock front. Both $v_{\mathrm{s}}$ and $v_{\mathrm{f}}$ depend strongly on the EOS used. If a density isomer (cf figure 3) exists, then the shock disappears at some density and reappears again at higher densities. This behaviour should clearly be seen in experiments, when the achieved density is varied with the bombarding energy (cf also section 4.1, figure 20).

\section{Modelling of heavy-ion reactions}

In this section we introduce the basic models that are used to describe heavy-ion collisions theoretically. Because of their conceptional differences we distinguish between two branches of model. The models in the first branch describe the reaction by macroscopic, thermodynamical quantities, i.e. density, energy and entropy and will therefore be called macroscopic. Those in the second branch describe the reaction by following microscopically the trajectories of each single nucleon; these models we shall call microscopic. 


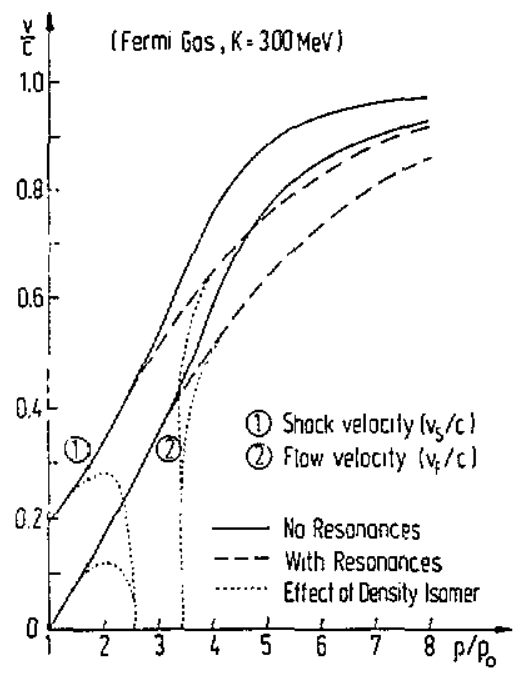

Figure 8. The dependence of the velocity of the shock front $v_{\mathrm{S}}$ and the matter flow velocity $v_{\mathrm{F}}$ on the density: broken curves, influence of the hadronjc resonances; dotted curves, influence of a density isomer at $\varrho=3 Q 0$ (Scheid et al 1974).

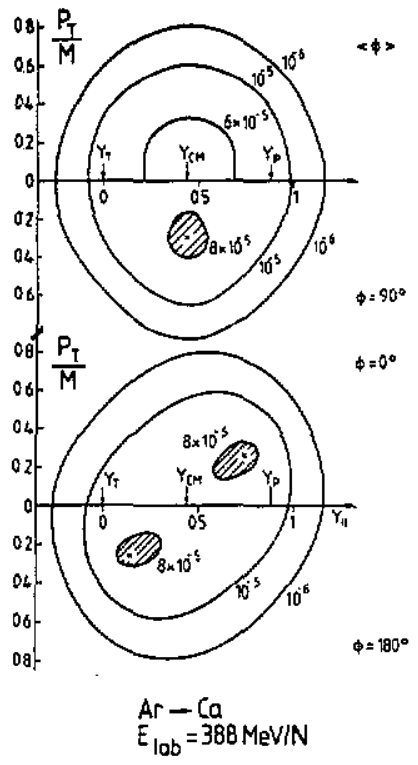

Figure 9. The triple differential invariant cross section $(1 / E)\left(\mathrm{d}^{3} \sigma / \mathrm{d} p^{3}\right)$ is shown for the reaction $\mathrm{Ca}$ $(400 \mathrm{~A} \mathrm{MeV}, b=2 \mathrm{fm})+\mathrm{Ca}$ as obtained from a fluid dynamical calculation (Buchwald et al 1983).

\subsection{The macroscopic models}

We will start our survey with a short description of the nuclear fluid dynamics (NFD) model, since this model has been influential in the development of the theory of heavy-ion reactions since its conception. Let us therefore start with a brief comparison of the assumptions and basic features of this group of models.

The validity of the NFD models is based on instantaneous local equilibrium, which will certainly hold only approximately in realistic situations. The criteria for the applicability of the NFD models therefore are (i) the system must have many degrees of freedom; and (ii) the mean free path $(\lambda)$ of the nucleons must be short compared with the dimension $(D)$ of the system. For peripheral collisions (and also for central collisions of light nuclei) the average number of nucleon-nucleon collisions is $\langle n\rangle \approx D / \lambda \approx 1$ and the hydrodynamical approach is not applicable. However, for central collisions the average number of collisions per nucleon is larger than one, in particular if high compressions are achieved. Thus the best chance of the NFD models to be applicable is clearly in central collisions of the heaviest nuclei.

Already it becomes clear here that the mean free path of the nucleons is the key quantity for the applicability of all macroscopic models. Simple kinetic models estimate the mean free path from $\lambda=1 /(\varrho \cdot \sigma)$ where $\sigma$ is the nucleon-nucleon cross section. This yields a short mean free path at low energies, and it was claimed (Sobel et al 1975) that, due to the decreasing $\mathrm{N}-\mathrm{N}$ cross section, $\lambda$ will increase with bombarding energy and therefore the shock compression mechanism will no longer be valid. Hence it is of basic importance to test the NFD approach by extracting the mean free path of the nucleons either from first principles or from more sophisticated models. Here the microscopic models present an ideal 
tool, since they follow the trajectory of each nucleon individually and can therefore be used to 'measure' $\lambda$ (cf also section 4.2 and figure 28 ).

One of the great advantages of the NFD model is that the nuclear matter properties, i.e. the nuclear EOS and dissipative coefficients (such as viscosity and thermoconductivity) are used directly as input. This makes it very easy to use the NFD model as a testing ground for the influence of the EOS and dissipative processes on the reactions. Furthermore the NFD model is conceptionally well defined and thus is of great importance as a reference model.

Although NFD is a well defined approach for the description of massive heavy ions, it is not a complete description of a heavy-ion collision. It can be used to describe the compression and expansion stage of the reaction, but then, when the matter expands and all the $\mathrm{N}-\mathrm{N}$ collisions cease, a stage is reached, where the system is so dilute that the basic assumptions of the NFD approach are no longer valid. At this point additional assumptions have to be made in order to describe the 'freeze-out' of this homogeneous, dilute system into an inhomogeneous sample of particles $(p, n, \pi, d, t, \alpha, \ldots)$, which will then move on without further interaction.

The equations of motion for a non-relativistic, viscous fluid can be written as a system of five continuity equations (indices ocurring twice are to be summed over):

$$
\begin{aligned}
& \frac{\partial \varrho}{\partial t}+\frac{\partial}{\partial x_{i}}\left(\varrho v_{i}\right)=0 \\
& \frac{\partial\left(\varrho v_{i}\right)}{\partial t}+\frac{\partial}{\partial x_{j}}\left(\varrho v_{i} v_{j}\right)=\frac{\partial}{\partial x_{j}} P_{i j}-\varrho \frac{\partial \Psi}{\partial x_{i}} \\
& \frac{\partial \varepsilon}{\partial t}+\frac{\partial}{\partial x_{i}}\left(\varepsilon v_{i}\right)=\frac{\partial}{\partial x_{j}}\left(v_{i} P_{i j}\right)-\frac{\partial q_{i}}{\partial x_{i}}-\varrho v_{i} \frac{\partial \Psi}{\partial x_{i}}
\end{aligned}
$$

where $\varrho, \varrho v_{i}$ and $\varepsilon=\varrho\left[m v^{2} / 2+W(\varrho, T)\right]$ are the local densities for baryon number, momentum and energy, respectively. $v_{i}$ is the local velocity and $q_{i}=-\kappa \partial T / \partial x_{i}$ is the vector of heat transport according to Fourier's law, where $\kappa$ is the coefficient of thermal conductivity. Yukawa and Coulomb potentials are considerd by $\Psi$.

The stress tensor $P_{i j}$ is defined by

$$
P_{i j}=-p \delta_{i j}+\eta\left(\frac{\partial v_{i}}{\partial x_{j}}+\frac{\partial v_{j}}{\partial x_{i}}-\frac{2}{3} \delta_{i j} \frac{\partial v_{k}}{\partial x_{k}}\right)+\xi \delta_{i j} \frac{\partial v_{k}}{\partial x_{k}}
$$

with $\eta$ and $\xi$ being the bulk and shear viscosity coefficients. Substituting this expression into equation (20) and neglecting the heat conduction $(\kappa=0)$ yields the well-known NavierStokes equations, which reduce in the absence of viscosity $(\eta=\xi=0)$ to the Euler equations.

The first calculations solved the Euler equations numerically for the one fluid case (Stöcker et al 1979, 1980a, 1981, 1982, Nix and Strottman 1981, Buchwald et al 1984). The energy and angular distributions were calculated from the particle density and velocity vectors at a time sufficiently long, so that the residual thermal energy is negligible, i.e. the densities are low.

During the expansion thermal equilibrium can only be maintained until the fluid reaches a break-up density $\left(\varrho_{B U} \approx 0.3-0.7 \varrho_{0}\right)$. Then the system breaks up into free particles, which reach the detectors with the momentum distributions they had in the freeze-out moment. The implementation of this freeze-out concept is the main difference between the different NFD approaches. It has been shown that the proper treatment of this break-up stage improves 
the agreement with the data (Stöcker et al 1981). One of the first predictions of the NFD model was the sideward emission of nuclear matter, due to shock compression (Scheid et al 1974, Baumgardt et al 1975), implying considerable transformation of the incident kinetic energy into compression and thermal energy. This sideward emission was proposed to be even more pronounced for heavier fragments, due to less thermal distortion (Baumgardt et al 1975). Quite early experiments (Baumgardt et al 1975, Baumgardt and Schopper 1979) supported this hypothesis; preferential sideward emission of mainly $\alpha$ particles was observed in the irradiation of $4 \pi$ particle detectors (emulsion, $\mathrm{AgCl}$ detectors) with light nuclei at $E_{\mathrm{Lab}}=200-400 \mathrm{AMeV}$, when selecting nearly central collisions. This was interpreted as being due to a strong, sidewards travelling compression wave, called the Mach shock wave. The first three-dimensional, non-relativistic, non-viscous, one-fluid dynamical calculations revealed this sidewards fiow in asymmetric heavy-ion collisions (Stöcker et al 1979, 1980a) in contrast to the oversimplified geometrical fireball (Westfall et al 1976, Gosset et al 1977, 1978, Das Gupta and Lam 1979, Myers 1978) and intranuclear cascade models (Yariv and Fraenkel 1979, 1981, Stevenson 1978, 1980). The first impact parameter averaged data on light-fragment emission did not show sideward peaked angular distributions (Sandoval et al 1980, Nagamiya et al 1981); thus they seemed to support the oversimplified models. However, these data turned out to be extremely inadequate, in fact wrong. When the first high multiplicity selected data for exclusive reactions became available (Stöcker et al 1980, Beavis et al 1983, Gustafsson et al 1984) they confirmed the theoretically predicted sideward peak in the distributions of flow angles in an event-by-event analysis (see also section 4.1). As a typical prediction of an NFD model we show in figure 9 the triple differential invariant cross section $(1 / E)\left(\mathrm{d}^{3} \sigma / \mathrm{d} p^{3}\right)$ for the reaction $\mathrm{Ca}(400 \mathrm{~A} \mathrm{MeV}, b=7 \mathrm{fm})+\mathrm{Ca}$. One clearly observes the in-plane flow $\left(\Phi=0^{\circ}, 180^{\circ}\right)$ for particles close to beam rapidities as well as the out-of-plane squeeze $\left(\Phi=90^{\circ}\right)$ of midrapidity nucleons. This out-of-plane squeeze has experimentally been observed only very recently (Gutbrod et al 1989b, 1990a, b, Schmidt 1991), by a new analysis of the Plastic Ball data.

These three-dimensional NFD calculations neglected the influence of the nuclear viscosity and thermal conductivity. Therefore previous one (Csernai et al 1980, Csernai and Barz 1981) and two-dimensional viscous calculations (Buchwald et al 1981) have been extended to the first fully three-dimensional viscous hydrodynamical treatment of heavy-ion collisions (Buchwald et al 1983a). One of the basic assumptions of the NFD models, the concept of local equilibrium, is clearly not fulfilled in heavy-ion collisions, especially in the highly anisotropic initial stage of the reaction. In order to remove this problem a non-relativistic three-fluid model (Csernai et al 1982, Rosenhauer et al 1987) was developed, in which two of the three fluids represent the target and projectile and the third fluid represents the thermalized nucleons. It was expected that the assumptions of the NFD models would be fulfilled in these three fluids.

Because of the 'increasing' mean free path of the nucleons at higher energies, it was supposed that at least some nucleons would traverse the entire target and punch through. For this reason, the relativistic (see equation (16)) two-fluid model was introduced, where one assumes that the projectile and target stream through each other and collectively decelerate (Amsden et al 1977a, b, Ivanow et al 1985, Clare and Strottman 1986, Mishustin et al 1989, Satarov 1990).

As a final point in this section we want to mention some early, very simplified models which are based on thermodynamical concepts. These models have been stimulated by the participant-spectator picture, combined with thermodynamic concepts. Considering a collision between two nuclei, the participant-spectator picture distinguishes between three different components. Those parts of the nuclei which do not belong to the overlap region 
between the projectile and target (i.e. the volume which is not cut out of the projectile and target when they are supposed to move through each other on straight lines) are called spectators, while the nucleons in the overlap region are called participants. Only the participants are supposed to undergo any reactions. The first models which have been developed from this concept are the fireball (Westfall et al 1976, Gosset et al 1977, 1978), the firestreak (Das Gupta and Lam 1979, Myers 1978) and the row-on-rows (Hüfner and Knoll 1977) models. These models describe the physics of the participant component only and are supposed to give the spectrum of the nucleons in the CM frame of the participant region which is here called the fireball. The original fireball model treats this region as a single, equilibrated object while the later forms of this model (firestreak, row-on-rows) treat the geometry in a more sophisticated way, by dividing up the participant region into several colliding tubes which are handled separately. The basic assumptions of these models is the applicability of the participant-spectator geometry and the assumption of global equilibrium in the whole fireball, streaks or rows, respectively.

\subsection{The microscopic models}

After this short overview of the macroscopic models, which have been-and still are-used to describe the gross features of heavy-ion collisions, we switch over to the microscopic models, that have been developed in order to compare to experimental data in much more detail.

As already mentioned, we denote as microscopic all those models that in some way follow the trajectories of each single nucleon. Such a microscopic description of a heavyion collision also gives us a unique opportunity to test the extent of which the macroscopic approaches are valid, i.e. whether the mean free path of the nucleons is small compared with the radius of the system and whether a local, or even a global, equilibrium is achieved. Since it is beyond the scope of this article to describe the whole zoo of models that are (or have been) on the market, we will give a short overview of the most important ones, which have been developed in the last two decades and then give a detailed description of the two surviving models that have been extensively used to describe heavy-ion collisions, i.e. the Vlasov-Uehling Uhlenbeck (VUU) and the quantum molecular dynamic (QMD) model.

If one wants to construct a realistic theory of heavy-ion collisions one should, in principle, describe the simultaneous interaction between relativistic, quantum mechanical wave packets via the concept of nucleon-nucleon interactions for a scattering inside a medium. This task, however, is so complicated that it has not even been touched upon in its full scheme so far. So one has to make several approximations to the quantal $n$-body system.

Two extreme pictures can be obtained if one assumes that the nuclei interpenetrate each other and only interact by

(i) binary hard collisions; or

(ii) the superposition of the soft interactions of all nucleons.

In the first case, the inter nuclear cascade model (INC), the important quantum effects have been incorporated by using experimental nucleon-nucleon scattering cross sections. The disadvantage, however, is the complete absence of compression effects. This type of model has been widely used to describe heavy-ion collisions (Bondorf et al 1976, Gudima and Toneev 1978, Stevenson 1978, 1980, Yariv and Fraenkel 1979, 1981, Gudima et al 1979, Halbert 1981, Cugnon et al 1981, 1982, Kitazoe et al 1984). In the second case, the so called equation of motion (EOM) or molecular dynamics (MD) models, one treats the N-N interaction purely classical via two-body interactions and integrates the Newtonian EOMS numerically (Bodmer and Panos 1977, Wilets et al 1977, 1978, Bodmer et al 1980, Kiselev 
and Pokroskil 1983, Kiselev 1984, 1986, Molitoros et al 1984, Schlagel and Pandharipande 1987. Beauvais et al 1987). This, however, has the great disadvantage that all the quantum effects are neglected and classical potentials can only provide a poor approximation to $\mathrm{N}-\mathrm{N}$ scattering and nuclear binding properties.

A better treatment of the quantum properties of nucleons is given by solving the timedependent Schrödinger equation in the Hartree-Fock approximation. This time-dependent Hartree-Fock model (TDHF) has been applied with some success to heavy-ion collisions at low energies (Koonin 1975, Bonche et al 1976, Stöcker 1980b, 1983, Aichelin and Stöcker 1985), and has the great advantage that it treats explicitly the Fermionic aspects of the nucleons but, since collisions have to be neglected, it fails to reproduce heavy-ion data at higher energies $\left(E_{\text {Lab }} \gg 10 \mathrm{AMeV}\right)$.

We have seen that the INC model is unrealistic since it neglects the soft interaction and the classical molecular dynamics (MD) model fails due to the neglect of hard collisions and quantum effects. The next two sections are therefore denoted to the description of two types of model which combine the basic features of the INC and MD models.

\subsubsection{The Vlasov-Uehling-Uhlenbeck (VUU) model. A transport equation which contains} both a mean field and hard $\mathrm{N}-\mathrm{N}$ collisions is the so called Vlasov-Uehling-Uhlenbeck (VUU) equation (Kruse et al 1985a, b, Aichelin and Bertsch 1985, Stöcker and Greiner 1986, Molitoris et al 1987, 1988a, b). It has also been called Boltzmann-Uehling-Uhlenbeck (BUU) (Bertsch et al 1984) or Landau-Vlasov (Gregoire et al 1987) equation. These three manifestations of this model solve the same equation with different numerical techniques. For particles in an external field this approach was first developed by Nordheim (1928) and Uehling and Uhlenbeck (1933). The derivation of the vUU equation can be found in almost all review articles (see, e.g., Bertsch and Das Gupta (1988) and Aichelin (1991)) and textbooks and will therefore not be repeated here.

The vuU equation can be understood as a transport equation for the one-particle distribution function $f(r, p, t)$

$$
\begin{aligned}
\frac{\partial f}{\partial t}+\frac{p}{m} \cdot \frac{\partial f}{\partial x} & -\nabla U \frac{\partial f}{\partial p}=\int \frac{\mathrm{d}^{3} p^{\prime} \mathrm{d}^{3} p_{1} \mathrm{~d}^{3} p_{1}^{\prime}}{(2 \pi)^{6}} \\
& \times \sigma \mid v-v_{1} ! f_{1}^{\prime} f^{\prime}\left(1-f_{1}^{\prime}\right)\left(1-f^{\prime}\right)-f_{1} f(1-f)\left(1-f_{1}\right) \delta\left(p+p_{1}+p^{\prime}+p_{1}^{\prime}\right) .
\end{aligned}
$$

The vuU equation contains, on the left-hand side, a mean-field potential $U$ and, on the right-hand side, the collision integral with the Pauli blocking factors $(1-f)$ and the $\mathrm{N}-\mathrm{N}$ scattering cross section $\sigma$. The Fermi-Dirac distribution function, which is a solution of the left-hand side (also called the Vlasov equation when the right-hand side is equal to zero) is the equilibrium solution of the collision term. The basic ingredients in the vUU model are therefore:

(i) the long-range $\mathrm{N}-\mathrm{N}$ interaction via the mean field $U(\varrho)$;

(ii) the short-range $\mathrm{N}-\mathrm{N}$ interaction via hard collisions with the differential cross section $\mathrm{d}^{2} \sigma / \mathrm{d} \Omega \mathrm{d} E$;

(iii) the Pauli exclusion principle via the blocking factors $(1-f)$.

For the mean-field potential $U(\varrho)$ a local Skyrme interaction is commonly used. The Skyrme interaction can be written as a potential

$$
\hat{V}=\sum_{i j} \hat{V}_{i j}^{(2)}+\sum_{i j k} \hat{V}_{i j k}^{(3)}
$$


with two- and three-body components. In configuration space the two-body part is given by

$$
\begin{aligned}
\hat{V}^{(2)}\left(\boldsymbol{r}_{i}-\boldsymbol{r}_{j}\right)= & t_{0}\left(1+x_{0} \hat{P}_{\sigma}\right) \delta\left(\boldsymbol{r}_{i}-\boldsymbol{r}_{j}\right)+\frac{1}{2} t_{1}\left[{\hat{k^{\prime}}}^{2} \delta\left(\boldsymbol{r}_{i}-\boldsymbol{r}_{j}\right)+\delta\left(\boldsymbol{r}_{i}-\boldsymbol{r}_{j}\right) \hat{k}^{2}\right] \\
& +t_{2} \hat{k^{\prime}} \delta\left(\boldsymbol{r}_{i}-\boldsymbol{r}_{j}\right) \hat{k}+\mathrm{i} W_{0} \hat{k^{\prime}} \delta\left(\boldsymbol{r}_{i}-\boldsymbol{r}_{j}\right) \hat{\sigma} \cdot \hat{k}
\end{aligned}
$$

where

$$
\hat{k}=\left(\frac{\partial}{\partial r_{i}}-\frac{\partial}{\partial r_{j}}\right) / 2 i \quad \hat{k}^{\prime}=\hat{k}^{\dagger} \quad \hat{P}_{\sigma}=\frac{1}{2}\left(1+\sigma_{i} \cdot \sigma_{j}\right) .
$$

The three-body part is given by:

$$
\hat{V}^{(3)}\left(r_{i}, r_{j}, r_{k}\right)=t_{3} \delta\left(r_{i}-r_{j}\right) \delta\left(r_{i}-r_{k}\right)
$$

This Skyrme interaction is usually used in the Hartree-Fock method, where the nuclear ground state is represented by a Slater determinant of single-particle states $\Psi$. The expectation value of the total energy is then given by

$$
E=\langle\Psi|\hat{T}+\hat{V}| \Psi\rangle=\int H(r) \mathrm{d}^{3} r
$$

In infinite nuclear matter this reduces to

$$
\frac{E}{A}=\frac{H}{\varrho}=\frac{3}{5} E_{\mathrm{F}}+\frac{3}{8} t_{0} \varrho+\frac{1}{16} t_{3} \varrho^{2}+\frac{3}{80}\left(3 t_{1}+5 t_{2}\right) \varrho k_{\mathrm{F}}^{2}
$$

and the potential is given by $(U=\partial H / \partial \varrho)$

$$
U(\varrho)=\frac{3}{4} t_{0} \varrho+\frac{3}{16} t_{3} \varrho^{2}+\frac{3}{80}\left(3 t_{1}+5 t_{2}\right) \varrho k_{\mathrm{F}}^{2} .
$$

Neglecting the momentum-dependent effects (they will be reintroduced later in a phenomenological way) this leads to a mean-field potential of the form

$$
U(e)=\alpha^{\prime} Q+\beta^{\prime} e^{\gamma}
$$

where we have already generalized the quadratic three-body term in order to vary also the nuclear compression constant $K$ as well (see also section 2). The $\mathrm{N}-\mathrm{N}$ cross section on the right-hand side of equation (22) is assumed to be the free $\mathrm{N}-\mathrm{N}$ cross section, which is taken from experiments. Since the exact dependence of $\sigma_{N N}$ on the surrounding medium is not known, this cross section can be treated as a parameter (it should, however, be fixed independently from theoretical nuclear matter calculations, see e.g., Haar and Malfliet (1986, 1987), Ohtsuka et al (1987)). The vUU equation is difficult to solve directly, since it is an highly nonlinear integro-differential equation in six-dimensional phase space. The semiclassical character of this equation, however, allows it to be solved in terms of quasiparticles, whose mean positions are solutions of Newton's equations (Molitoris et al 1984, 1988a, b, Kruse et al 1985a, b).

To solve the VUU equation the nucleons are represented as a sum of point-like test particles

$$
f_{i}\left(r_{i}, p_{i}, t\right)=\sum_{i=1}^{\bar{N} A} \delta\left(r_{i}-r_{i 0}\right) \cdot \delta\left(p_{i}-p_{i 0}\right)
$$


where $A$ denotes the number of nucleons and $\tilde{N}$ the number of test particles per nucleon. Then $\tilde{N}$ parallel collisions are followed and the phase-space density $f$ is computed as an ensemble average of the test particles in a sphere around each test particle. The local gradient of the field is computed via a finite difference method between the two half-spheres of this sphere. Each test particle in one of the parallel events is allowed to collide with the other test particles (of the same parallel event) with their free $\mathrm{N}-\mathrm{N}$ cross section.

Let us now briefly list the basic ingredients and assumptions of the vUU model (and all the other models which solve the vur equation).

(i) The derivation of the Vlasov equation from the $N$-body Liouville equation requires a dilute gas limit, i.e. $\lambda>a$, where $\lambda$ is the mean free path of the nucleons and $a$ the scattering length.

(ii) The $\mathrm{N}-\mathrm{N}$ interaction is divided into a long-range attractive part and a short-range repulsive part which are treated separately.

(iii) Subsequent collisions are independent, i.e. off-shell collisions are not allowed.

(iv) The vuU equation is a single-particle equation. The computation via parallel events destroys all $\mathrm{N}-\mathrm{N}$ correlations and therefore makes it impossible to treat correlations and the fragmentation in heavy-ion collisions.

3.2.2. The $Q M D$ model. We have seen in the previous section that the vUU model provides a useful tool to describe the one-particle observables of heavy-ion reactions. However, phenomena such as fragment formation can hardly be described in such a singleparticle theory, since they are intimately connected to many-body correlations. The correct propagation of correlations is lost in the vUU model, in contrast to the MD models, by using the test particle method to obtain an ensemble average of the one-particle distribution function $f$. If one wishes to treat correlations exactly, one has to avoid the test particle method. For this reason in the QMD model a single Gaussian is used to describe the nucleons. So each nucleon is described by a boosted Gaussian wave packet with fixed width

$$
\Psi_{i}\left(r_{i}, t\right)=\frac{1}{(2 \pi L)^{3 / 2}} \exp \left[\frac{\mathrm{i}}{\hbar} p_{i} \cdot r_{i}-\frac{1}{2 L}\left(r_{i}-r_{i 0}(t)\right)^{2}\right]
$$

In the numerical calculations it is more convenient to use the corresponding Wigner density

$$
f_{i}\left(r_{i}, p_{i}, t\right)=\frac{1}{(\pi \hbar)^{3}} \exp \left\{-\frac{\left(r_{i}-r_{i 0}(t)\right)^{2}}{2 L}-\left(p_{i}-p_{i 0}(t)\right)^{2} \frac{2 L}{\hbar^{2}}\right\}
$$

where $r_{i 0}$ and $p_{i 0}$ are the centroids of particle $i$ in coordinate and momentum space.

The phase-space distribution can now be expressed as

$$
f(r, p, t)=\sum_{i=1}^{N} f_{i}(r, p, t)
$$

In terms of these Gaussians the baryon density is given by

$$
\varrho_{\mathrm{B}}(r, t)=\sum_{i=1}^{N} \int f_{i}(r, p, t) \mathrm{d}^{3} p=\frac{1}{(2 \pi L)^{3 / 2}} \sum_{i=1}^{N} \exp \left[-\frac{\left(r-r_{i 0}\right)^{2}}{2 L}\right]
$$


With knowledge of the phase-space distribution it is possible to calculate thermodynamical quantities locally. The ensemble average of a macroscopic quantity $\chi(r, t)$ is given by

$$
\langle\chi(r, t)\rangle=\frac{1}{\rho(\boldsymbol{r}, t)} \int \mathrm{d}^{3} p \chi(\boldsymbol{p}, \boldsymbol{r}, t) f(\boldsymbol{p}, \boldsymbol{r}, t) .
$$

In the basic version of the QMD model we used a local two- and three-body Skyrme interaction (cf equations (23),(24)) and a Coulomb and Yukawa interaction.

With the Gaussian distributed nucleons (equation (31)) one calculates the following Hamiltonian function.

$$
\begin{aligned}
& H=\sum_{i=1}^{N}\left(\sqrt{p_{i 0}^{2}+m^{2}}-m\right)+\frac{1}{2} \sum_{\substack{i, j=1 \\
j \neq i}}^{N} \int f_{i}\left(r_{i}, p_{i}\right) V_{\text {Yuk }}\left(r_{i}, r_{j}\right) f_{j}\left(r_{j}, p_{j}\right) \mathrm{d}^{3} r_{i} \mathrm{~d}^{3} r_{j} \mathrm{~d}^{3} p_{i} \mathrm{~d}^{3} p_{j} \\
& +\frac{1}{2} \sum_{\substack{i, j=1 \\
j \neq i}}^{N} \int f_{i}\left(\boldsymbol{r}_{i}, \boldsymbol{p}_{i}\right) V_{\text {Coul }}\left(\boldsymbol{r}_{i}, \boldsymbol{r}_{j}\right) f_{j}\left(\boldsymbol{r}_{j}, \boldsymbol{p}_{j}\right) \mathrm{d}^{3} r_{i} \mathrm{~d}^{3} r_{j} \mathrm{~d}^{3} p_{i} \mathrm{~d}^{3} p_{j} \\
& +\frac{1}{2} \sum_{\substack{i, j=1 \\
j \neq i}}^{N} \int f_{i}\left(r_{i}, p_{i}\right) V_{\mathrm{Sk}}^{(2)}\left(r_{i}, r_{j}\right) f_{j}\left(r_{j}, p_{j}\right) \mathrm{d}^{3} r_{i} \mathrm{~d}^{3} r_{j} \mathrm{~d}^{3} p_{i} \mathrm{~d}^{3} p_{j} \\
& +\frac{1}{2} \sum_{i=1}^{N} \frac{1}{3} \sum_{\substack{j=1 \\
j \neq i}}^{N} \sum_{\substack{k=1 \\
k \neq j, k \neq i}}^{N} \int f_{i}\left(\boldsymbol{r}_{i}, \boldsymbol{p}_{i}\right) f_{j}\left(\boldsymbol{r}_{j}, \boldsymbol{p}_{j}\right) f_{k}\left(\boldsymbol{r}_{k}, \boldsymbol{p}_{k}\right) V_{\mathrm{Sk}}^{(3)}\left(\boldsymbol{r}_{i}, \boldsymbol{r}_{j}, \boldsymbol{r}_{k}\right) \\
& \times \mathrm{d}^{3} r_{i} \mathrm{~d}^{3} r_{j} \mathrm{~d}^{3} r_{k} \mathrm{~d}^{3} p_{i} \mathrm{~d}^{3} p_{j} \mathrm{~d}^{3} p_{k} .
\end{aligned}
$$

Evaluating the integrals yields

$$
\begin{aligned}
& H=\sum_{i=1}^{N}\left(\sqrt{p_{i 0}^{2}+m^{2}}-m\right)+\frac{1}{2} \sum_{\substack{i, j=1 \\
j \neq i}}^{N} \frac{V_{\text {Yuk }}^{0}}{2\left|r_{i 0}-r_{j 0}\right|} \exp \left[L / \gamma_{\text {Yuk }}^{2}\right] \\
& \times\left\{\exp \left[-\left|\boldsymbol{r}_{i 0}-\boldsymbol{r}_{j 0}\right| / \gamma_{\left.\gamma_{\mathrm{uk}}\right]}\right]\left[1-\operatorname{erf}\left(\frac{\left(2 L / \gamma_{Y \mathrm{uk}}\right)-\left|\boldsymbol{r}_{i 0}-\boldsymbol{r}_{j 0}\right|}{\sqrt{4 L}}\right)\right]\right. \\
& -\exp \left[+\left|r_{i 0}-r_{j 0}\right| / \gamma_{\text {Yuk }}\left[1-\operatorname{erf}\left(\frac{\left(2 L / \gamma_{Y u k}\right)+\left|r_{i 0}-r_{j 0}\right|}{\sqrt{4 L}}\right)\right]\right\} \\
& +\frac{1}{2} \sum_{\substack{i, j=1 \\
j \neq i}}^{N} \frac{e^{2} / 4}{\left|r_{i 0}-r_{j 0}\right|} \operatorname{erf}\left(\frac{\left|r_{i}-r_{j}\right|}{\sqrt{4 L}}\right) \\
& +\sum_{i=1}^{N}[\frac{\alpha}{2} \underbrace{\frac{1}{(4 \pi L)^{3 / 2} \varrho_{0}} \sum_{\substack{j=1 \\
j \neq i}}^{N} \exp \left[-\frac{\left(r_{i 0}-r_{j 0}\right)^{2}}{4 L}\right]}_{Q_{1}\left(r_{i 0}\right)} \\
& \left.+\frac{\beta}{\gamma+1}\left(\frac{1}{(4 \pi L)^{3 / 2} \varrho_{0}} \sum_{\substack{j=1 \\
j \neq i}}^{N} \exp \left[-\frac{\left(r_{i 0}-r_{j 0}\right)^{2}}{4 L}\right]\right)^{\gamma}\right] .
\end{aligned}
$$


The first term denotes the kinetic energy of the centroids of the nucleons; the second and third term are the Yukawa and Coulomb interactions, respectively, for Gaussian shaped wave packets (31) of the nucleons. The last term represents the Skyrme part of the interaction which can, for infinite nuclear matter, be considered as a density-dependent interaction. Note, however, the difference between $\varrho_{\mathrm{I}}$ in equation (36) and $\varrho_{\mathrm{B}}$ in equation (33). Only for infinite systems are these two terms equal.

The parameters $\alpha, \beta$ and $\gamma$ are adjusted to reproduce the properties of infinite nuclear matter, i.e.

$$
\begin{aligned}
& \left.\frac{E}{A}\right|_{\varrho=\varrho_{0}}=-16 \mathrm{MeV} \\
& P=\left.\varrho^{2} \frac{\partial E / A}{\partial \varrho}\right|_{\varrho=\varrho_{0}}=0 \mathrm{MeV} \mathrm{fm}^{3} \\
& K=\left.9 \varrho^{2} \frac{\partial^{2} E / A}{\partial \varrho^{2}}\right|_{\varrho=\varrho_{0}}= \begin{cases}200 \mathrm{MeV} & \text { (soft EOS) } \\
380 \mathrm{MeV} & \text { (hard EOS). }\end{cases}
\end{aligned}
$$

In order to obtain a reasonable simulation of finite nuclei, we adjust the two Yukawa parameters $V_{\text {Yuk }}$ and $\mathcal{K}_{\text {Yuk }}$ (the Yukawa potential also gives a density-dependent contribution to the EOS, which must be taken into account when adjusting the parameters).

It has recently been emphasized (Aichelin and Stöcker 1986, Gale et al 1987, Bodmer and Panos 1977, Bodmer et al 1980) that non-equilibrium effects will play an important role in a realistic treatment of heavy-ion collisions. The most pronounced effect can be expected from the momentum dependence of the nuclear interaction (cf equation (23)) which leads to an additional repulsion between the nucleons. For the computation of such momentumdependent interactions (MDI) we parametrized the momentum dependence of the real part of the optical p-nucleus potential, in order to substitute the term proportional to $k_{\mathrm{F}}^{2}$ in the Skyrme interaction, which is in striking contrast to the data above $E_{\text {Lab }} \approx 150 \mathrm{AMeV}$.

We get

$$
U_{\mathrm{MDI}}=\delta \ln ^{2}\left[\epsilon^{\prime}\left(p_{i}-p_{j}\right)^{2}+1\right] \delta\left(r_{i}-r_{j}\right)
$$

with $\delta=1.57 \mathrm{MeV}$ and $\epsilon^{\prime}=5 \times 10^{-4} \mathrm{MeV}^{-2}$.

For infinite nuclear matter at zero temperature we can relate the square of the relative momenta to the density using the Fermi gas approximation and we obtain for the potential

$$
U_{\mathrm{MDI}}=\delta \ln ^{2}\left[\epsilon\left(\varrho / \varrho_{0}\right)^{2 / 3}+1\right] \varrho^{\prime} / \varrho_{0}
$$

(with $\epsilon=21.54 \mathrm{MeV}$ ), which gives after integration over $\varrho\left(H=(1 / \varrho) \int U(\varrho) \mathrm{d} \varrho\right)$ the corresponding term in the Hamiltonian.

When using the MDI, one has to readjust the parameters of the forces in order to yield the same nuclear matter properties as these without MDI (see table 1).

In the later versions of the QMD model the averaged charge in the Coulomb term of equation (36) is replaced by an explicit Coulomb interaction of the form

$$
V^{\text {Coul }}\left(r_{i}-r_{j}\right)=\frac{e^{2}}{\left|r_{i}-r_{j}\right|} \sigma_{i} \cdot \sigma_{j}
$$

where $\sigma_{i}$ is equal to 1 for protons and 0 for neutrons. In this case an asymmetry interaction of the following form was also incorporated:

$$
V_{\mathrm{a}}\left(\boldsymbol{r}_{i}-\boldsymbol{r}_{j}\right)=\Omega \delta\left(\boldsymbol{r}_{i}-r_{j}\right) \cdot 2\left(\sigma_{i}-\frac{1}{2}\right) \cdot 2\left(\sigma_{j}-\frac{1}{2}\right)
$$


which yields after integration an asymmetry energy of the form

$$
H_{\mathrm{a}}=\sum_{i=1}^{N} \Omega\left(\varrho_{i}^{\prime}(\mathrm{p})-\varrho_{i}^{\prime}(\mathrm{n})\right)
$$

Now the numerical simulation of the collision takes place in three steps. First the projectile and target are initialized in their rest frames. Therefore one determines the positions of the nucleons randomly in a sphere of radius $R=1.14 \mathrm{fm} A^{1 / 3}-0.3 \mathrm{fm}$, but requiring, in addition, a minimum distance of $1.5 \mathrm{fm}$ between each pair of nucleons. This procedure yields a smooth distribution in configuration space. The momenta of the nucleons are then randomly chosen in the interval $\left.0 \leqslant|p| \leqslant \mid p_{\mathrm{F}}\right\}$, where the local Fermi momentum $p_{\mathrm{F}}$ is determined from the local density via the Fermi gas approximation. A minimum distance $\Delta p=180 \mathrm{MeV}^{-1}$ is required for each spin-isospin identical pair in order to fulfil the Pauli principle $a b$ initio.

Successfully initialized nuclei are then boosted towards each other with the proper centre of mass velocities using relativistic kinematics. The centroids of each Gaussian are then propagated corresponding to the Hamilton equations with the Hamiltonian of equation (36) in a leap frog integration routine with time steps ranging from 0.2 to $0.4 \mathrm{fm} c^{-1}$.

After each integration step the hard $\mathrm{N}-\mathrm{N}$ collisions are treated in the same way as in the VUU and INC models via a stochastic scattering term. Two nucleons can scatter if the spatial distance of the centroids of their Gaussians is smaller than $\sqrt{\sigma_{\text {tot }} / \pi}$. The energy and angular distributions of the experimental differential scattering cross section $\mathrm{d}^{2} \sigma / d \sqrt{s} \mathrm{~d} \Omega$ are reproduced on average. Inelastic collisions lead to the formation of delta particles, which can be reabsorbed in the inverse reaction. We do not incorporate free (s-wave) pions here, unlike the vUu model. Whenever a collision occurs, we check the phase-space distribution around the final states of the scattering partners. We determine the ratios $P_{1}, P_{2}$ of the final phase spaces which are already occupied by other nucleons. The collision is then blocked with the probability

$$
P_{\text {Block }}=\min \left[1, P_{1} \cdot P_{2}\right]
$$

Whenever a collision is blocked, the momenta of the scattering partners are replaced by the values they had prior to the scattering.

In order to demonstrate the importance of Pauli blocking, the fraction of Pauli blocked collisions to all attempted collisions is shown in figure 10 for the system $\mathrm{Nb}+\mathrm{Nb}$ for different beam energies between 50 and $1050 \mathrm{AMeV}$. The ratio is plotted against $t \cdot \beta$. Here $t \cdot \beta$ is the scaled reaction time, i.e. $t$ multiplied by the velocity $\beta$ of the incoming projectile in an equal speed system. This product corresponds to the distance travelled by the projectile and target in the $z$ direction. It scales the time according to the velocity of the incoming projectile.

All curves start with a blocking fraction of one. This is due to the fact that all collisions are Pauli blocked in the ground state. After the two nuclei touch on another the blocking factors decrease and saturate after $t \cdot \beta \approx 5-6 \mathrm{fm}$. This distance corresponds to the total overlap of both nuclei. For very low bombarding energies (the TDHF regime) the blocking factor remains close to one, whereas for higher energies it decreases down to 0.2 at $1 \mathrm{~A} \mathrm{GeV}$.

Next we want to show how the QMD model compares with previously developed singleparticle models. Therefore we display in figure 11 the time evolution of the density profile of the reaction $\mathrm{C}(84 \mathrm{AMeV}, b=1 \mathrm{fm})+\mathrm{C}$, calculated with three different theories, namely the quantum mechanical TDHF method, the classical Vlasov equation (VUU without 
$\mathrm{Nb}(b=3$ fm, Soft EOS $)+\mathrm{Nb}$

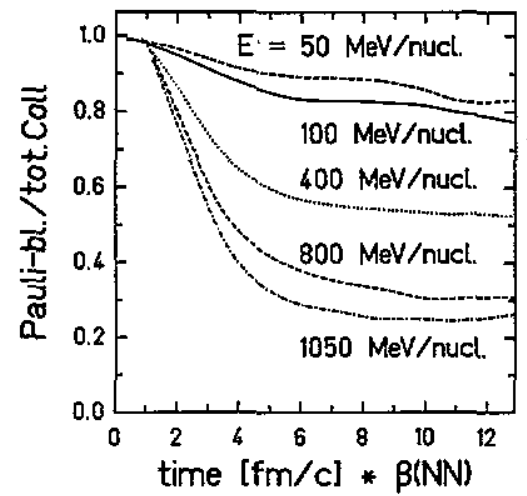

Figure 10. Time dependence of the ratio of Pauli blocked collisions to the total number of attempted collisions at different beam energies for the system $\mathrm{Nb}$ $+\mathrm{Nb}$ at $b=3 \mathrm{fm}$.

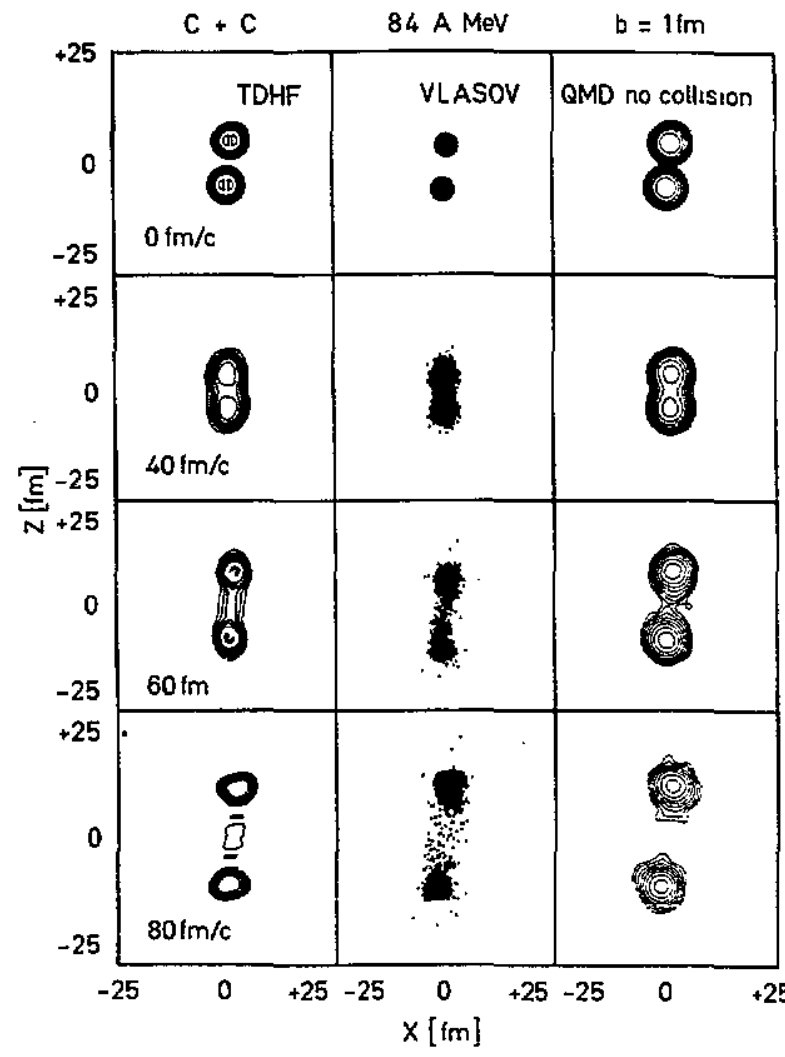

Figure 11. Comparison of the different mean-field calculations from the reaction $\mathrm{C}$ $(84 \mathrm{AMeV}, b=1 \mathrm{fm})+\mathrm{C}$. The density profiles are displayed for the results of the TOHF, Vlasov and the QMD approach without collisions (Aíchelin et at 1989).

collisions) and the QMD without collisions. We observe a striking similarity between all these theories. The longitudinal momentum transfer, as well as the momentum transfer in the transverse direction, are very similar in all these theories. The similarity of these results indicates that details of the initializations and the detailed form of the wavefunction of the nucleons are of minor importance for the time evolution of the system.

The time evolution of this system changes, however, dramatically if the collisions are incorporated into the VUU and QMD approaches. A detailed comparison between the VUU/BUU and the QMD results is shown in figure 12 for the same reaction. The double differential cross section $\mathrm{d}^{2} \sigma / \mathrm{d} E \mathrm{~d} \Omega$ for protons agrees well in those models. We can 
therefore conclude that the QMD approach reproduces the results obtained with the VUU/BUU approach, if single-particle observables are considered. In addition, the mean-field version reproduces the time evolution of the Vlasov and TDHF calculations.

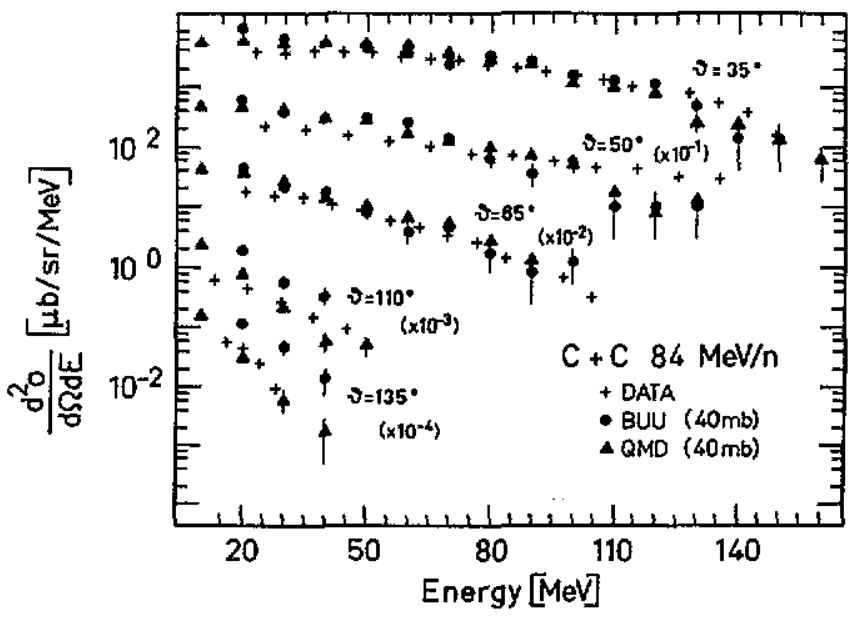

Figure 12. Comparison of the double differential cross section $\mathrm{d}^{2} \sigma / \mathrm{d} E$ $d \Omega$ obtained with the suU and QMD approach for the inclusive reaction $\mathrm{C}(84 \mathrm{AMeV})+\mathrm{C}$ (Aichelin et al 1989).

Before we investigate in detail the physics of heavy-ion reactions we briefly want to describe some modifications to the original QMD model which have recently been developed in order to remedy two of the main shortcomings of the model.

One deficiency in the model is that the nuclear ground state cannot be simulated in a reasonable fashion, because of the absence of the Fermi motion, generated by the Pauli exclusion principle.

Wilets et al $(1977,1978)$ and Callaway et al $(1979)$ suggested that the Pauli exclusion principle might be simulated by a momentum-dependent repulsive potential. Later on several forms of this potential were introduced, yielding a reasonable reproduction of the gross thermostatic properties (Dorso and Randrup 1988, Boal and Glosli 1988, Boal et al 1989, Peilert et al 1991).

We employ the Gaussian Pauli potential introduced by Dorso and Randrup $(1988,1989)$ and Peilert (1991). With such a potential the total energy of the 'free' Fermi gas is given by

$$
\begin{aligned}
E_{\mathrm{tot}}^{\mathrm{FG}}=E_{\mathrm{kin}}+ & E_{\mathrm{Pau}}=\sum_{i}\left(\sqrt{p_{i 0}^{2}+m^{2}}-m\right)+\frac{1}{2} \sum_{i \neq j} V_{\mathrm{Pau}}^{0}\left(\frac{\hbar}{q_{0} p_{0}}\right)^{2} \\
& \times \exp \left[-\frac{r_{i j}^{2}}{2 q_{0}^{2}}-\frac{p_{i j}^{2}}{2 p_{0}^{2}}\right] \delta_{\tau_{,} \tau_{j}} \delta_{\sigma_{i} \sigma_{j}}
\end{aligned}
$$

where $\sigma_{i}$ and $\tau_{i}$ denote the spin-isospin index of nucleon $i$.

In the following we show how this potential reproduces the Fermi energy in an infinite Fermi gas. We parametrized the Pauli potential such that the total energy $E_{\mathrm{tot}}^{\mathrm{FG}}$ matches the kinetic energy of the free Fermi gas.

The thermostatic properties are obtained by averaging over several hundred statistically distributed manifestations of the system, sampled by means of the Metropolis importance sampling method (Metropolis et al 1953) on the basis of the appropriate weight

$$
w \sim \mathrm{e}^{-E_{\mathrm{tal}}^{\mathrm{FO}} / T} .
$$


Figure 13 shows the calculated energy per nucleon. For comparison, the full curves show the corresponding exact values for a free Fermi gas. In all cases the overall agreement is rather satisfactory. In particular the ground-state configuration no longer collapses in the limit $T \rightarrow 0$.

free fermigas

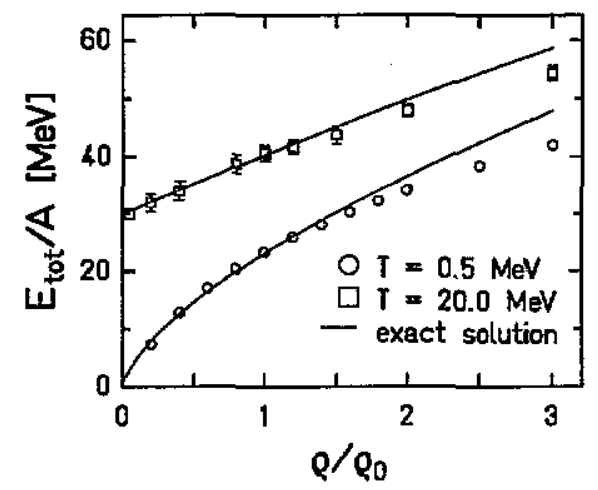

Figure 13. The exact results for the energy per nucleon in a free Fermi gas is shown by the full curves, while the symbols indicate the calculated results using the Pauli potential (Peilert et al 1991).

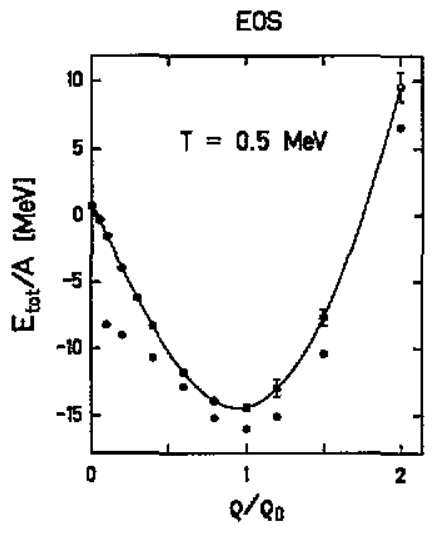

Figure 14. Clusterization energy: the energy per nucleon is shown as a function of the specified density at the temperature $T=0 \mathrm{MeV}$. The open circles were obtained when the initial random positions of the nucleons were kept fixed while the full circles result when the positions are also free (Peilert et al 1991).

Next we want to investigate how far the treatment of the Fermionic aspects of the nuclei modifies the EOS for infinite nuclear matter. Therefore we employ the full Hamiltonian of the QMD model (see equation (36)) plus this Pauli potential and do a Metropolis sampling for a system for $4 \times 54$ particles in a box with periodic boundary conditions.

Figure 14 shows the resulting EOS for cold nuclear matter $(T=0.5 \mathrm{AMeV})$ for two different cases. First we kept the coordinates of the nucleons fixed, in order to achieve a homogenous density and performed the Metropolis sampling only in momentum space (full curve). Then we performed the Metropolis sampling in the full six-dimensional phase space (open symbols). As can be seen in this case the resulting energies for subsaturatiuon densities are considerably lower, since the nuclei cluster into finite systems and gain the binding energy. Therefore the ground state at these densities is highly inhomogeneous. This fact has to be considered if one performs thermostatic calculations, i.e. one observes phase transitions in this density regime.

The implementation of the Pauli potential in the dynamical QMD model yields two huge improvements. First the ground states are now well defined and the Metropolis procedure can be used to initialize the projectile and target in their real ground states, which yields a much improved stability for the initialized nuclei. Second the excitation energy of the resulting fragments can then be determined with respect to their true ground state and can be used to describe the long-term behaviour (evaporation, multifragmentation or fission) of those fragments in an independent model. We will come back to this point in section 5 .

The parameters of the several manifestations of the QMD model can be found in table 1 .

Recently Feldmeier introduced an antisymmetrized molecular dynamics version called the fermionic molecular dynamics (FMD) (Feldmeier 1990). In this framework the manybody wavefunction is described by a Slater determinant of the single-particle Gaussian 
Table 1. Parameters of the interaction used in the QMD model.

\begin{tabular}{|c|c|c|c|c|c|}
\hline & \multicolumn{3}{|c|}{$\begin{array}{l}\text { No Pauli potential } \\
\text { no MDr }\end{array}$} & \multirow{2}{*}{$\begin{array}{l}\text { No Pauli potential } \\
\text { with MDI } \\
K=200 \mathrm{MeV}\end{array}$} & \multirow{2}{*}{$\begin{array}{l}\text { Pauli potential } \\
\text { no } \mathrm{MDl} \\
K=380 \mathrm{MeV}\end{array}$} \\
\hline & $K=380$ & $\mathrm{MeV}$ & $K=200 \mathrm{MeV}$ & & \\
\hline$\overline{V_{\text {Pau }}^{0}(\mathrm{MeV})}$ & - & & - & - & 99.5 \\
\hline$q_{0}(\mathrm{fm})$ & - & & - & - & 3.0 \\
\hline$p_{0}\left(\mathrm{MeV} \mathrm{c}^{-1}\right)$ & - & -- & 一 & - & 120.0 \\
\hline$L\left(\mathrm{fm}^{2}\right)$ & 2.165 & & 2.165 & 2.165 & 2.165 \\
\hline$\alpha(\mathrm{MeV})$ & -124.0 & & -356.0 & -390.0 & -84.56 \\
\hline$\beta(\mathrm{MeV})$ & 70.5 & & 303.0 & 320.0 & 188.18 \\
\hline$\gamma$ & 2.0 & & $7 / 6$ & $8 / 7$ & 1.457 \\
\hline$V_{\text {Yuk }}^{0}(\mathrm{MeV})$ & -10.0 & & -10.0 & -10.0 & -85.0 \\
\hline$\gamma_{\text {Yuk }}(\mathrm{fm})$ & $\mathrm{I} .5$ & & 1.5 & 1.5 & 1.0 \\
\hline$\Omega(\mathrm{MeV})$ & - & & - & - & 25.0 \\
\hline$\varepsilon\left(\mathrm{MeV}^{-2}\right)$ & - & & - & 21.54 & - \\
\hline$\delta(\mathrm{MeV})$ & - & & - & 1.57 & - \\
\hline
\end{tabular}

wavefunctions and the time evolution of the parameters of these Gaussians is determined by a time-dependent variational principle (Koonin 1975, Kramer and Saraceno 1981).

This model has not yet been solvable in its full complexity, but restricted versions of the FMD model have recently been applied to heavy-ion collisions (Konopka 1991, Valta 1991, Horiuchi 1991, Valta et al 1992, Ono et al 1992).

\section{The testing ground for the nuclear EOS}

The main purpose for which all the models, that have been introduced in section 3 , have been developed is to investigate the nuclear EOS by comparing the model results with experimental data. For this purpose it is not sufficient to perform high-quality experiments and to have the most efficient theories with which to compare them, it is also of the utmost importance to find the appropriate observables, i.e. ones that are sensitive to the underlying EOS. The modelling of heavy-ion collisions gives us a unique opportunity to change the physical input (e.g. the EOS and the $\mathrm{N}-\mathrm{N}$ cross sections) in order to investigate to what extent the results depend on these quantities. Then one has to find observables that are directly connected to one input parameter, without being disturbed by other effects. These observables can then be used to adjust the input parameters independently by comparing them with experiments. It is this deep interplay between theory and experiment which makes this field so interesting for many physicists.

For testing the influence of the EOS on the observables, the NFD and VUU models are the most promising, since they use the EOS, via a density-dependent mean field, directly as input. So it is easy to change the EOS and even to implement exotic forms (e.g. an EOS with secondary minima as was shown in figure 3). This is more difficult in the QMD approach, since there the EOS enters only indirectly. The direct inputs into the QMD model are the nucleon-nucleon interactions, which, in infinite nuclear matter, lead to an EOS of the form $E / A=E_{\text {kin }}(\varrho, T)+\alpha\left(\varrho / \varrho_{0}\right)+\beta\left(\varrho / \varrho_{0}\right)^{\gamma}$. In the original version of the QMD model the Fermi gas energy has been taken for the kinetic part. After the inclusion of the Pauli potential the total energy can be calculated self-consistently. Up to now no attempts have been made to change the functional form of this EOS in the QMD model, but it is easy to change the parameters $\alpha, \beta$ and $\gamma$ (with the restriction that it reproduces the known nuclear matter properties), in order to change the stiffness of the EOS (cf figure 6). 
The QMD model, however, is most efficient to test, via multifragmentation break up, also the low density part of the EOS with its-supposed-liquid-gas phase transition. This is not only of importance for low-energy collisions, where this multifragmentation break up is observed in central reactions; at higher energies it is also necessary to use a model, which describes the many-body degrees of freedom if one wants to make a direct comparison with experimental data. The reason for this is that most of the key observables that are sensitive to the EOS also depend on the size of the measured particles. Therefore one has to use a model which also describes the formation of fragments even if one looks at one-body observables (we will discuss the multifragmentation channels of these reactions in detail in section 5).

In this section we shall introduce the most important observables that have been found to depend on the physical input.

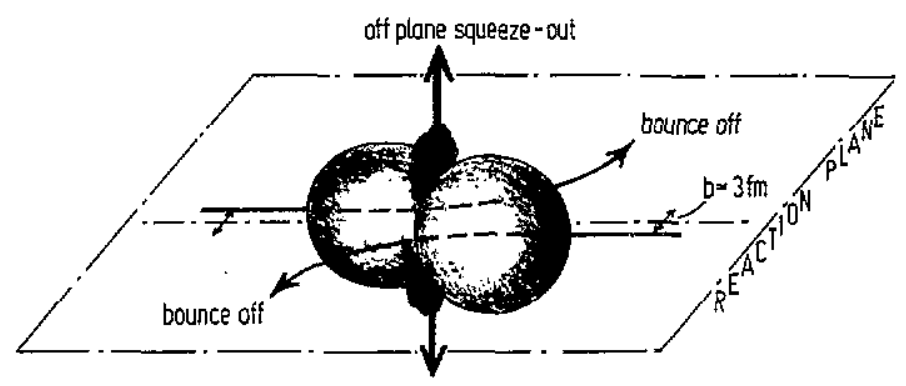

Figure 15. Pictorial representation of the in-plane bounce-off and out-of-plane squeeze-out of the participant matter in a heavy-ion collision.

\subsection{The collective flow}

The basic collective flow observables can be seen in figure 15. The picture one has in mind here is as follows. When nuclear matter has been stopped and compressed, it tries to escape and to expand under a finite angle (with respect to the beam axis). In the reaction plane this angle depends strongly on the impact parameter $b$ : for $b=0$ the flow of nuclear matter is perpendicular to the beam axis while for mid-central collisions the flow angle lies between $0^{\circ}$ and $90^{\circ}$. Perpendicular to the reaction plane, however, there is always a squeeze-out under $90^{\circ}$ in the $\mathrm{CM}$ frame. In this direction the matter can escape freely from the compression zone, without being disturbed by any spectator material or corona effects, i.e. it reflects the purely hydrodynamical part of the reaction. This effect corresponds to the classical push-out of matter discussed by Scheid et al $(1968,1974)$. One can already see from this schematic picture that all the important effects that one is looking for will strongly depend on the impact parameter and therefore the only way to measure these observables will be in an event-by-event analysis of highly exclusive experiments.

4.1.1. The in-plane bounce-off. One method to measure the collective flow is by global momentum analysis. The basic idea here is to measure event-by-event the momenta of all (charged) particles. Then one transforms into the $\mathrm{CM}$ frame and determines the direction of maximum momentum and energy flow. Similar problems in event topology analysis have been treated earlier in high-energy particle physics, later this so called sphericity analysis 
was proposed for the analysis of heavy-ion data (Cugnon et al 1982, Gyulassy et al 1982). The three-dimensional sphericity tensor is defined as

$$
F_{i k}=\sum_{v=1}^{A} \omega(v) \cdot p_{i}(v) p_{k}(v) \quad i, k=x, y, z
$$

$\omega(\nu)$ is a weight factor calculated event-by-event from the $\mathrm{CM}$ momenta of all measured particles. Choosing $\omega(\nu)$ in such a way that the composite particles obtain the same weight per nucleon as single nucleons with the same velocity, i.e. $\omega(v)=\frac{1}{2} m(v)$ leads to the kinetic energy flow tensor (Gyulassy et al 1982, Buchwald et al 1983b). Although being mathematically elegant, this method suffers substantially from finite particle number distortions for $A<100$, which allows only the maximum in the Jacobian free flow angle distribution $\mathrm{d} N / \mathrm{d} \cos \theta_{F}$, and not the mean values, to be extracted as a useful observable (Danielewicz and Gyulassy 1983).

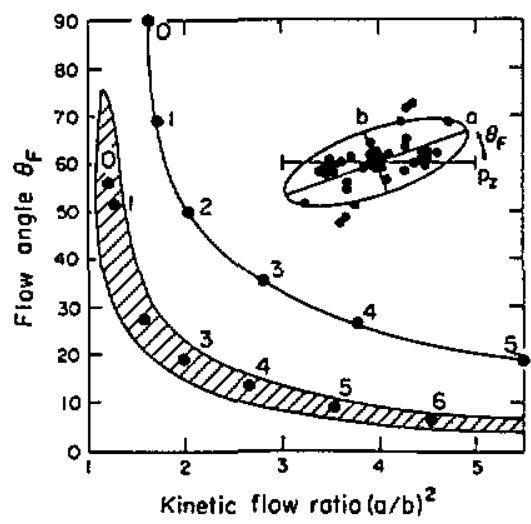

Figure 16. Kinetic energy flow analysis for the reaction $U(400 \mathrm{~A} \mathrm{MeV})+U$. The $R_{13}-\Theta$ plane for the INC model calculation (shaded area) and the NFD calculation (full curve) are shown. The numbers indicate the impact parameter (Buchwald et al 1983).

Figure 16 shows, for the reaction $U(400 \mathrm{AMeV})+\mathrm{U}$, the flow angle $\Theta_{\mathrm{CM}}^{\text {flow }}$, i.e. the angle of the largest principal axis of the kinetic energy flow tensor to the beam axis, against the aspect ratio $R_{13}\left(=\lambda_{1} / \lambda_{2}\right)$, where the $\lambda_{i}$ are the eigenvalues of $\hat{F}$ ordered according to $\lambda_{1}<\lambda_{2}<\lambda_{3}$. Here calculations done with the NFD model (full curve) are compared with INC calculations (hatched area). Note that $R_{13} \gg 1$ reflects events stretched in momentum space (high transparency) while $R_{13}=1$ corresponds to a spherical momentum distribution. This figure clearly indicates that the matter flow is more strongly correlated in the hydrodynamical calculation and that the flow angle increases with decreasing impact parameter.

The first experiment that has rigorously shown a non-zero flow, i.e. a $\mathrm{d} N / \mathrm{d} \cos \theta_{\mathrm{F}}$ distribution that is peaked at non-zero $\theta_{F}$ yalues, was performed by the Plastic Ball collaboration for the reaction $\mathrm{Nb}(400 \mathrm{~A} \mathrm{MeV})+\mathrm{Nb}$ (Gustafsson et al 1984) and later on also for heavier systems (Ritter et al 1985) (for a detailed discussion of these experiments see e.g., Kampert (1989), Gutbrod (1989a) and Schmidt (1991)).

Figure 17 shows the measured flow angle distributions for the systems $\mathrm{Ca}+\mathrm{Ca}$ and $\mathrm{Nb}$ $+\mathrm{Nb}$ at $400 \mathrm{~A} \mathrm{MeV}$ bombarding energy (Ritter et al 1985) compared with the INC (Yariv and Fraenkel 1979, Cugnon et al 1982, Cugnon 1980) and the NFD (Stöcker et al 1980a, Buchwald et al 1983b) calculations. A peak, different from zero, is only observed for the heavier system and in nuclear hydrodynamics calculations, while the cascade calculation fails to reproduce the collective flow, due to the absence of compression effects. 


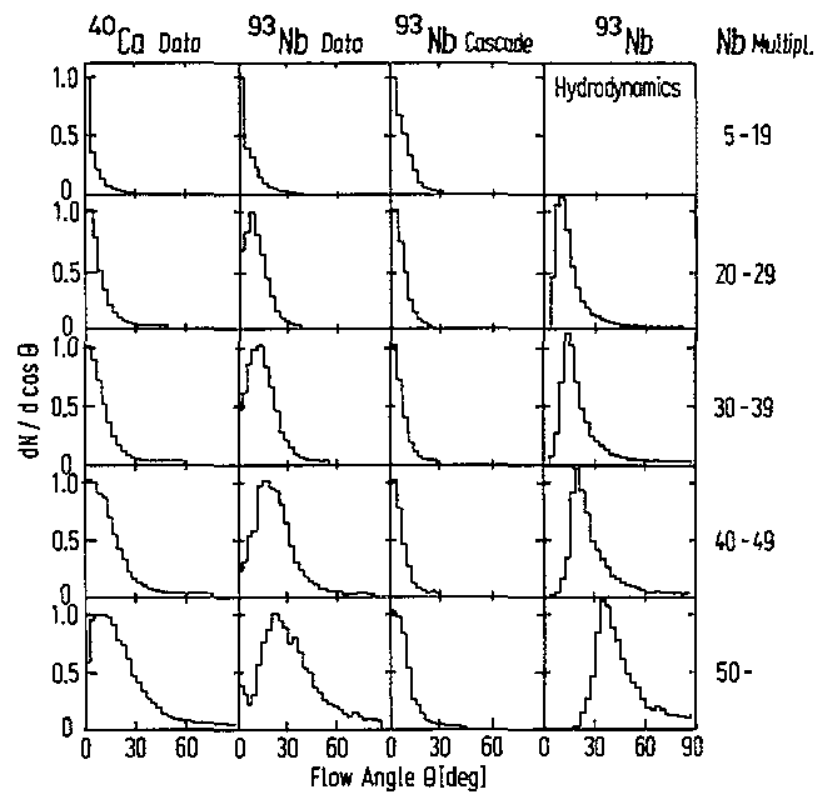

Figure 17. Distributions of the flow angles $\theta_{\mathrm{F}}$ for the reactions $\mathrm{Ca}+\mathrm{Ca}$ and $\mathrm{Nb}+\mathrm{Nb}$ at $400 \mathrm{~A} \mathrm{MeV}$ bombarding energy. The experimental data for the $\mathrm{Ca}$ and $\mathrm{Nb}$ case (Ritter et al 1985), compared with the theoretical calculations performed with the INC and NFD models for different multiplicity bins as indicated are shown.

This sphericity analysis has, however, the disadvantage that it constructs a global observable out of all measured particles from one event. So it is not very selective, since it also includes all the thermalized particles which smear out the important effects. For this reason this method cannot be used to extract any detailed information about the underlying physics.

In order to avoid these difficulties Danielewicz and Odyniec (1985) developed the so called global transverse momentum analysis. In this method the reaction plane is constructed for each event from the transverse momentum components of all particles. In the next step the transverse momentum vector of each particle is projected onto this plane, yielding the in-plane transverse momentum $p_{x}$, which is then usually plotted as a function of the Lorentz invariant rapidity $y=\frac{1}{2} \ln \left[\left(E+p_{\|}\right) /\left(E-p_{\|}\right)\right]$.

Figure 18 shows such a distribution for the measured system Au $(200 \mathrm{~A} \mathrm{MeV})+\mathrm{Au}$ (Gutbrod et al 1989a). This was the first exclusive experiment (in fact, until 1991 when the GSI $4 \pi$ spectrometer went into operation it was the only one), where the flow of composite particles was measured. The transverse momentum distributions for protons $(Z=1)$ are shown as well as for heavier fragments as indicated. One clearly observes the general behaviour of such distributions. The particles with rapidities close to the beam rapidity show a maximum flow, while for the stopped, thermalized particles at midrapidity, the transverse momentum vanishes due to the azimuthal symmetry (we will investigate later to what extent these particles are thermalized). This figure also shows clearly that a stronger flow effect is found for the heavier fragments, due to less thermal smearing as was proposed from early NFD calculations (Baumgardt et al 1975, Stöcker et al 1981).

For the comparison of the in-plane flow with theoretical calculations we use the QMD 


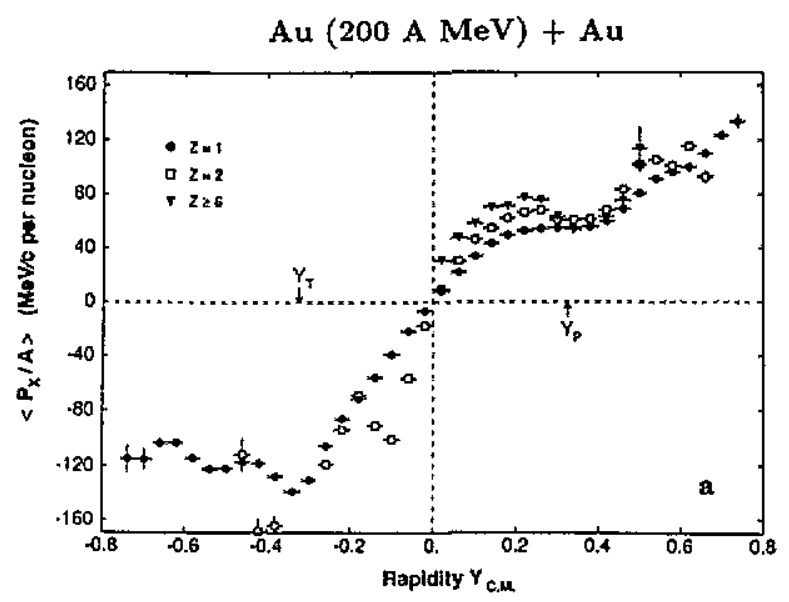

Figure 18. The mean value of the transverse momentum projected onto the reaction plane as a function of the CM rapdity for the renction $\mathrm{Au}(200 \mathrm{~A} \mathrm{MeV}) \div \mathrm{Au}$. The values for $Z=1,2$ and $\geqslant 6$ are shown (Gutbrod et al 1989a).

model, with different parameter sets for the relevant interactions. First we vary the nuclear EOS by changing the compression constant $K$ from $200 \mathrm{MeV}$ (the resulting EOS is called soft) to $380 \mathrm{MeV}$ (hard EOS), without using the momentum-dependent interactions (MDI). As a third case we use the soft $\operatorname{EOS}(K=200 \mathrm{MeV}$ ) with the MDI (SM). In the fourth case we also change the nucleon-nucleon cross section. The effective value of the $\mathrm{N}-\mathrm{N}$ scattering cross section in the nuclear medium is still not known precisely. This cross section may be reduced, due to the Pauli blocking of intermediate scattering states (Haar and Malfliet 1986, 1987, Cugnon et al 1987, Bertsch et al 1987, Ohtsuka et al 1987). It is well known that the $\mathrm{N}-\mathrm{N}$ cross section in infinite nuclear matter is lowered compared with that in the free case. One part of this reduction results from the Pauli blocking of the final scattering states. This leads to a reduced cross section $\sigma_{\mathrm{NN}}^{\mathrm{UU}}$, which is already included in all the models which use the Uehling-Uhlenbeck collision term via the explicit blocking of the final scattering states. In a Dirac-Brueckner theory the $\mathrm{N}-\mathrm{N}$ scattering also includes the blocking of intermediate scattering states. This leads to an additional reduction of the cross section (Haar and Malfliet 1986, 1987), which, for the present study, is approximated by a simple reduction factor

$$
\sigma_{\mathrm{NN}}^{\mathrm{eff}} \approx 0.7 \sigma_{\mathrm{NN}}^{\mathrm{UU}}
$$

It should be noted that such a global reduction in the cross section certainly does not reproduce the physical situation exactly, but our purpose here is only to show the changes in the observables that should be expected if the cross section in the nuclear medium is substantially different from the free one.

Before a comparison with experiment, one has to include the experimental efficiency filter in the theoretical calculations. In order to do so, we applied the same low-energy cutoff at $35 \mathrm{~A} \mathrm{MeV}$ to all particles and counted the intermediate mass fragments (up to mass 20 ) only if they were emitted with an angle lower than $30^{\circ}$ in the laboratory frame. One also has to be careful to correlate the experimental multiplicity triggers with the impact parameters used in the calculations. By its definition the transverse momentum analysis fails to detect 
flow effects for very central collisions; because of symmetry it is always $p_{x}(b=0 \mathrm{fm})=0$. A clear maximum is experimentally observed in the multiplicity dependence $p_{x}\left(N_{p}\right)$ (see (Kampert 1989)) in the bins MUL3 and MUL4. An analogous maximum is observed in the calculated impact parameter dependence $p_{x}(b)$ at $b=3 \mathrm{fm}$. Data and theory can thus be directly confronted by comparing the $p_{x} / A$ values at the corresponding maxima. One also has to be careful about the exact definition of the collective flow. One possible definition is based on the slope of the $p_{x}(y)$ distribution at midrapidity (Doss et al 1986). For heavy fragments, however, the slope of the $p_{x}(y)$ distribution at midrapidity cannot be defined, because the yields are strongly peaked at projectile and target rapidity (Peilert et al 1988). Only a few fragments are found at midrapidity, except for the most central collisions $(b \leqslant 1 \mathrm{fm})$, where the $p_{x} / A$ values are small. There is only one way out of this Catch22 situation, namely to study the $p_{x} / A$ values at their maximum yield, i.e. close to the projectile and target rapidities.
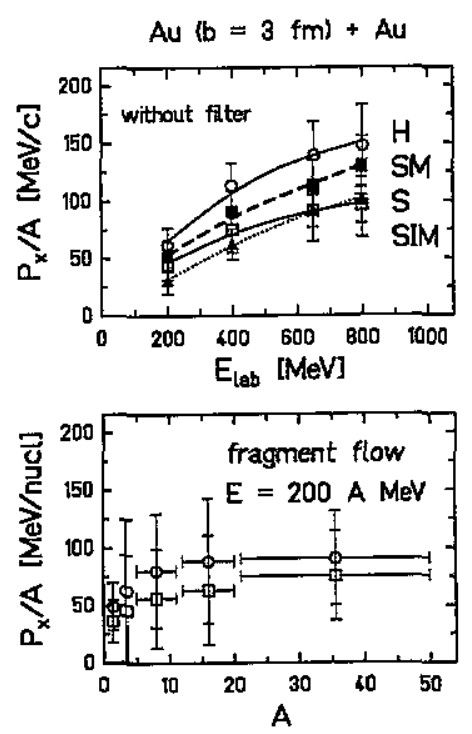

Figure 19. Excitation function of the flow (absolute value of the $p_{x}(y)$ distribution at projectile rapidity) for the reactions Au (200-800A MeV) + Au obtained with the QMD model with different interactions as indicated (upper part). The lower part shows the mass dependence of the fragment flow (Peilert et al 1989).

In figure 19 we show in the upper part the excitation function of this flow $\left(p_{x}\left(y_{p}\right) / A\right)$ for the singles $(A=1)$ calculated with the $\mathrm{QMD}$ model using the different interactions as indicated. One clearly observes a difference of about a factor two between the pure soft and hard EOS. This difference is, unfortunately, smeared out if one considers the other in-medium effects, e.g., the MDI and the effective nucleon-nucleon cross sections. The additionally included MDI increases the transverse flow so that the results obtained with a soft EOS plus MDI (SM) comes close to the hard EOS without MDI. The reduction of $\sigma_{\mathrm{NN}}$ acts in the opposite direction. Due to an increased transparency the flow decreases, when the cross section is lowered (see the curve labelled SM).

So we are left with the problem of disentangling the different influences, i.e. to find observables which are unambiguously influenced by one parameter only. With such observables at hand we can then fix all the unknown parameters independently.

The lower part of figure 19 shows the mass dependence of the fragment flow. Note that the flow of the intermediate mass fragments is more pronounced than the flow of the single nucleons. 
As one can see from the upper part of this figure the fow increases steadily with increasing bombarding energy, reflecting the increase in the compression energy deposited in the system. This, however, is only the case if the EOS shows an increase in the compression energy with increasing density. A drastic change in the excitation function of the flow is expected when the EOS shows any deviation from this scheme, for example in the form of a second minimum as was shown in figure 3 .

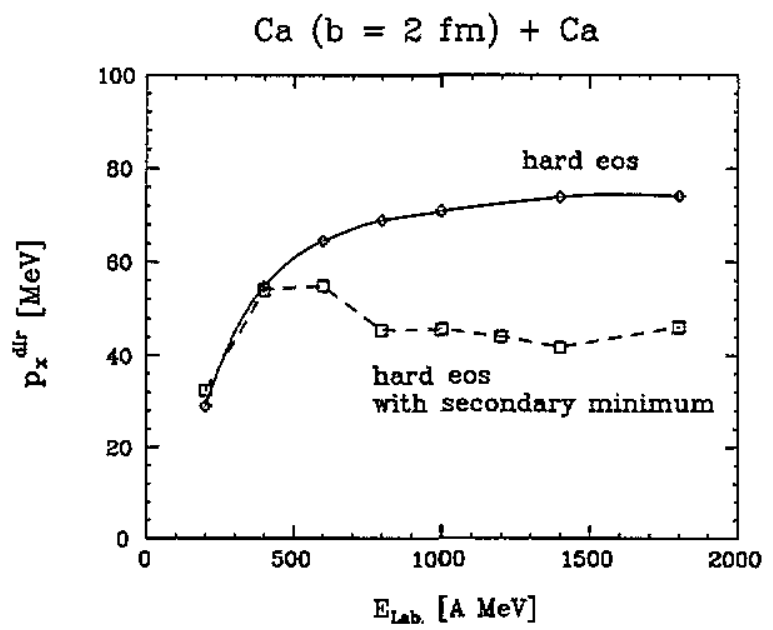

Figure 20. vuu calculation for the excitation function of the directed transverse flow $p_{x}^{\text {dir }}$ (from Hartnack (1992)). The upper curve was obtained using the hard Eos of figure 6 , while for the lower curve the exotic Eos with a secondary minimum at $Q \approx 2.5 Q_{0}$ was used (see the curve in figure 3 with $\alpha=1.0$ and $\beta=1.31$ ).

As an example for the consequences of such an exotic EOS we show in figure 20 the excitation function of the directed transverse flow $p_{x}^{\text {dir }}=\left.\sum_{i} p_{x}^{i}\right|_{y_{1}>y_{C M}}$ for the reaction $\mathrm{Ca}$ $(b=2 \mathrm{fm})+\mathrm{Ca}$. In this case a vuU calculation has been performed (Hartnack 1992) using the conventional hard EOS as well as the exotic EOS which was shown in figure 3 with the parameters $\alpha=1.0, \beta=1.35$. One clearly observes that, in contrast to the results obtained with the hard EOS the excitation function of the flow drops out when some critical density (bombarding energy) is reached and then decreases when the density (bombarding energy) in further increased. At the highest energies the excitation function starts to increase again. This behaviour will be even more pronounced for a larger system (e.g., Au + Au) but in this case we expect the drop-out to occur at energies larger than $1 \mathrm{~A} \mathrm{GeV}$ where one should use a relativistic modei.

Figure 21 shows the calculated (filled circles) and the measured (filled triangles) inplane transverse momenta distributions for the reaction $\mathrm{Au}(200 \mathrm{AMeV}, b=3 \mathrm{fm})+\mathrm{Au}$, evaluated with the Plastic Ball efficiency cuts for different fragment masses and for the soft and the hard EOS (without $\mathrm{MDI}, \sigma_{\mathrm{NN}}^{\mathrm{eff}}=\sigma_{\mathrm{NN}}^{\mathrm{IN}}$ ), respectively. Note again the increase in the $p_{x} / A$ values with the mass of the fragments both in theory and experiment, as well as the dependence of $p_{x} / A$ on the EOS. This difference between the results obtained with the soft and hard eOss is most pronounced for intermediate mass fragments. These fragments are formed early in the reaction as a result of the compression wave travelling through the 

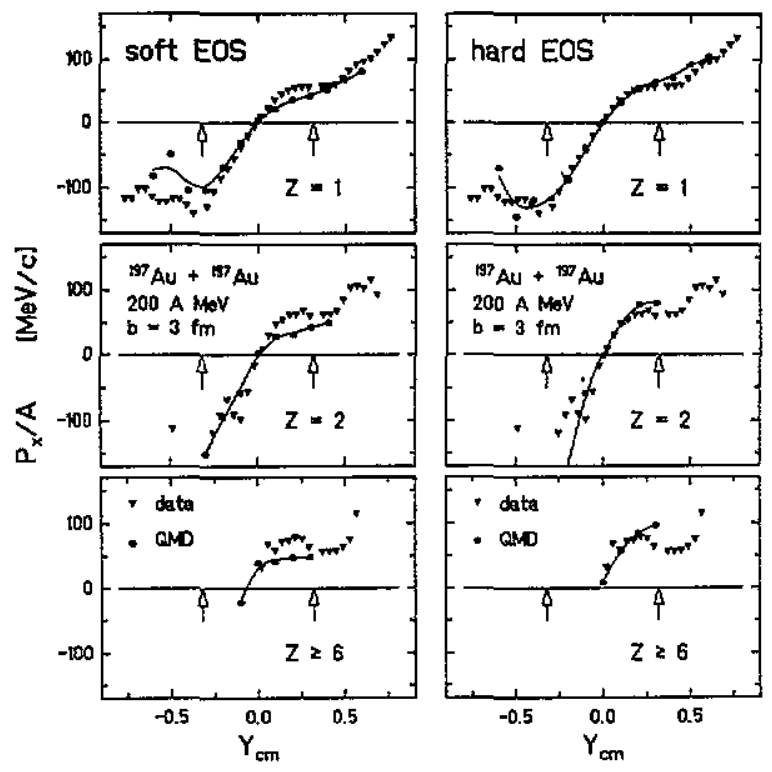

Figure 21. The transverse momentum distributions $p_{x}(y) / A$ of light and intermediate mass fragments obtained from the QMD calculations for the reaction $\mathrm{Au}(200 \mathrm{AMeV}, b=3 \mathrm{fm})+\mathrm{Au}$ are compared with the Plastic Ball data (full triangles) for different fragment classes as indicated. The left-(right-)hand column compares the results obtained with a soft (hard) EOS with the data Both the OMD results and the data have been taken at the impact parameter (multiplicity) where the transverse momentum shows a maximum (Peilert et al 1989).

system. The heaviest fragments $(A>30)$, on the other hand, are formed later in a decay chain from the excited projectile and target residue (see also section 5); hence they do not carry strong signatures from the compression stage.

With the invention of the SIS/ESR facility at the GSI in Darmstadt it then became possible to study these reactions (i.e. collisions of hecvy ions at high energies) in much more detail. One of the first experiments that was investigated there was the reaction $\mathrm{Au}$ $(150 \mathrm{~A} \mathrm{MeV})+\mathrm{Au}$ (Alard et al 1992). In figure 22 we therefore show the double differential invariant cross section $\left(1 / p_{\mathrm{t}}\right)\left(\mathrm{d}^{2} \sigma / \mathrm{d} y \mathrm{~d} p_{\mathrm{t}}\right)$, obtained with three different models, namely the INC, VUU and QMD models. In the case of the QMD model the results have been integrated over all nucleons, even if they were bound in clusters. Shown in this figure are the inclusive results (obtained from impact parameters $b \leqslant 6 \mathrm{fm}$ ) and the central collisions with $b<1 \mathrm{fm}$. In addition to this a directivity cut $D=\left|\sum_{i} p_{t}\right| /\left.\sum_{i}\left|p_{\mathrm{t}}\right|\right|_{y_{i}>y_{\mathrm{cm}}}<0.2$ has been applied to all events and to the central events only. First one observes that both the vUU and the QMD calculations yield almost the same distributions, as they should, because in this case a one-body observable is considered. The cascade calculation, however, clearly shows a different result. In contrast to the VUU and QMD results, which yield complete stopping, there is a considerable amount of transparency in the cascade calculation. The reason for this is again the absence of the compression effects, which has also led to the failure of the cascade models to explain the transverse flow.

Going from the inclusive distribution (upper row) to the more central triggers one observes a change in the momentum distributions. For the inclusive distributions the events look more or less thermal; this, however, is an artifact of the impact parameter mixing and not a physical observation. For the more central collisions one clearly observes that the 


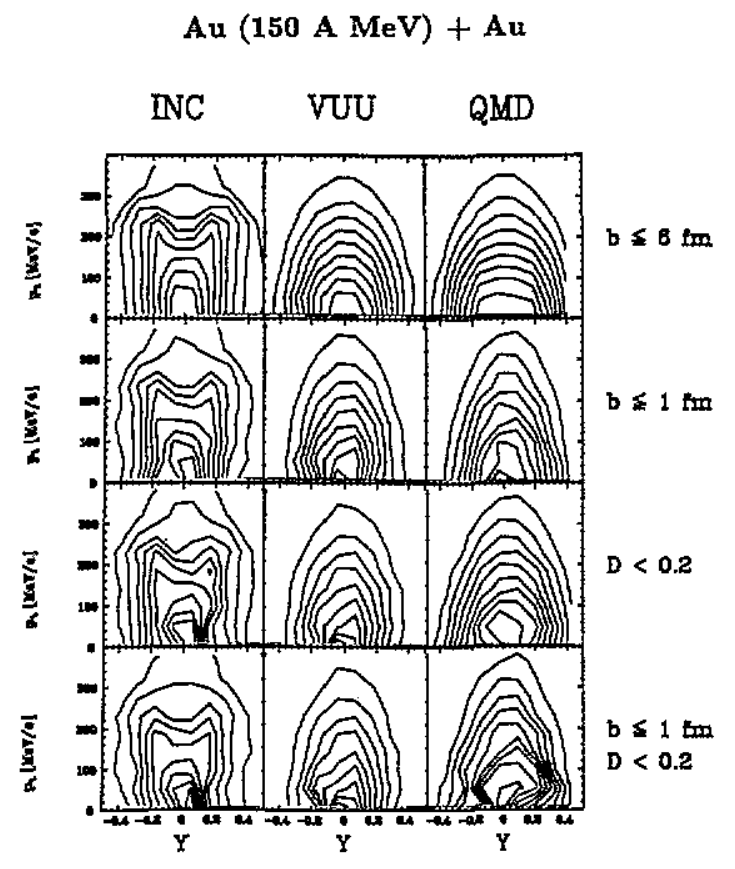

Figure 22. Double differential, invariant cross section $\left(1 / p_{t}\right)\left(d^{2} \sigma / d y d p_{t}\right)$ for the reaction Au $(150 \mathrm{AMeV})+\mathrm{Au}$, obtained with the INC, the vuU and the QMD model. The results are shown for the inclusive reaction $(b<6 \mathrm{fm})$ and for various other triggers on central collisions.

distributions are clearly not thermal, there is even a peak at finite transverse momenta for the most central collisions.

In parallel to the FOPI experiments at the GSI the EOS Time Projection Chamber (TPC) was developed in Berkeley. The EOS/TPC is a newly constructed detector that exceeds the capabilities of the Plastic Ball. The experiments with this detector have been devoted to measuring the excitation function of the collective flow effects up to the highest energies that are possible at the BEVALAC (Hjort et al 1994). It is our purpose to use the QMD model in order to perform a detailed comparison of the collective flow observables with the experimental data in the whole energy regime available at the BEVALAC. Since the experimental data are not yet fully analysed, we cannot present a comparison with QMD results right now. Figure 23 shows a prediction of the in-plane transverse flow $\left(p_{x}(y)\right)$ for the reaction $\mathrm{Au}+\mathrm{Au}$ at $400 \mathrm{~A} \mathrm{MeV}$ bombarding energy. The calculations have been done with the modified QMD model with and without the Pauli potential, using a compressibility constant of $380 \mathrm{MeV}$ for the impact parameter range $b=2-4 \mathrm{fm}$. The qualitative and quantitative results here agree well with the previous results, obtained with the original QMD version (see e.g. figure 19). In addition to the unfiltered results we also show, for the light fragments $(Z=1,2)$, the results that have been obtained after filtering the QMD events according to the EOS/TPC efficiencies. In this case we did not use the fixed impact parameter interval, but we used the same multiplicity trigger as the experimentalists (the MUL 3 bin is constructed in the same way as for the Plastic Ball data discussed earlier). One can see clearly from figure 23 that the Pauli potential affects the magnitude of the collective flow only slightly, also the filtering slightly lowers the flow.

We have seen that the absolute magnitude of the transverse flow is sensitive to the 
Bu (400 MeV, b=2-4fm) + Au
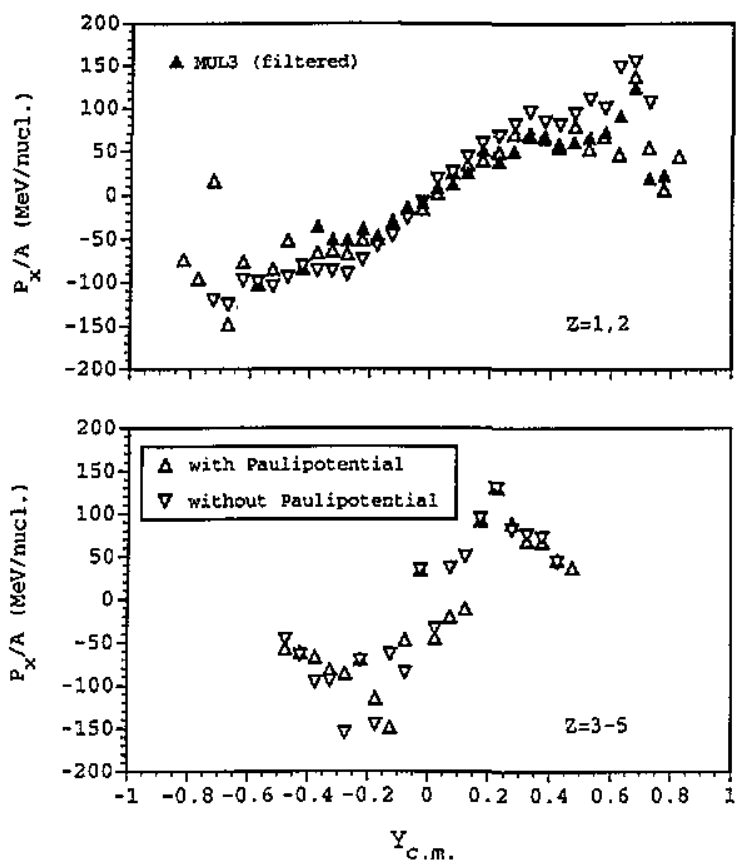

Figure 23. The in-plane transverse flow is shown for the reaction $\mathrm{Au}(400 \mathrm{AMeV}, b=2-4 \mathrm{fm}$ ) for fragments with $Z=1,2$ and $Z=3-5$. The upper figures also show results that have been obtained after filtering the QMD events according to the EOS/TPC efficiencies (labelled with MUL3). The open triangles show the results with and without the Pauli potential.
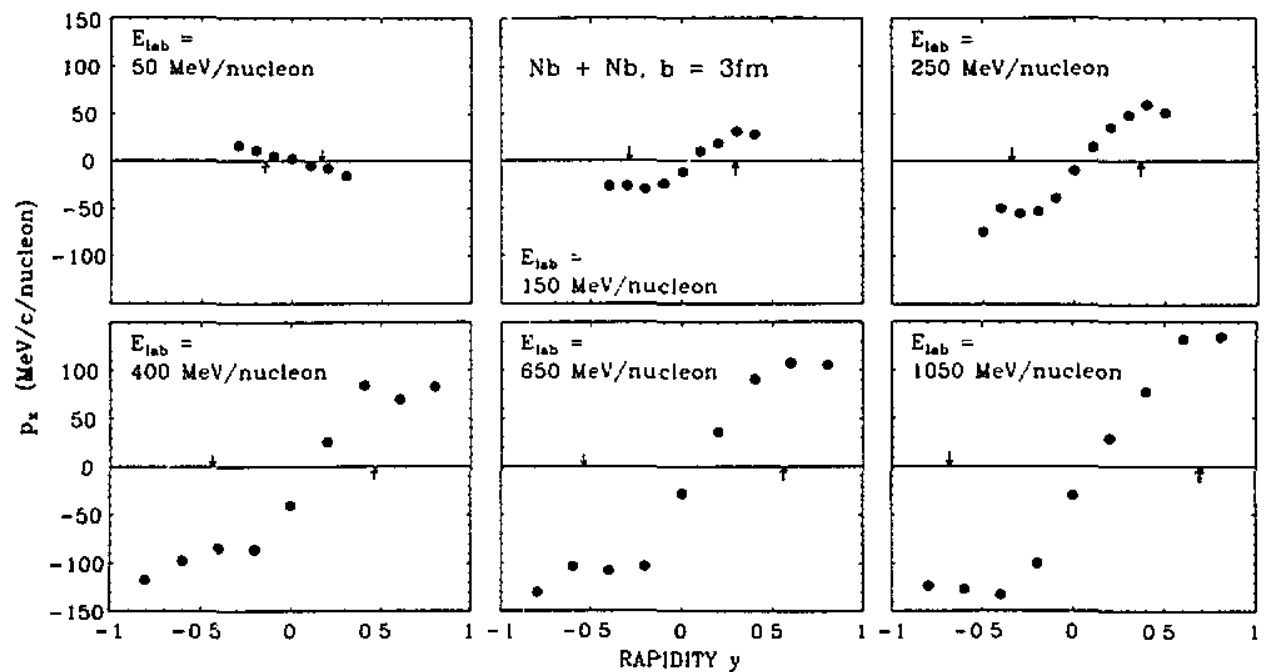

Figure 24. Transverse momentum distributions for the reaction $\mathrm{Nb}(b=3 \mathrm{fm})+\mathrm{Nb}$ obtained with the vuu model (Molitoris and Stöcker 1985). 
underlying Eos. This effect is more pronounced at high energies where higher compressions are obtained. But one can also use the same observable, i.e. the $p_{x}(y)$ distribution, to test the EOS at low densities. In figure 24 we present a vuU calculation (Molitoris and Stöcker 1985, Stöcker and Greiner 1986) for the reaction $\mathrm{Nb}(b=3 \mathrm{fm})+\mathrm{Nb}$. The calculated transverse momentum distributions are shown as a function of the beam energy. Observe that the $p_{x}$ values change sign in the energy regime between 50 and $100 \mathrm{~A} \mathrm{MeV}$. This is due to the fact that the attractive part of the nuclear interaction becomes dominant; the bounceoff, caused by the short-range repulsion at higher energies is converted into the negative angle defiection known from TDHF calculations in the same energy regime (Stöcker et al $1980 \mathrm{~b}$ ). This onset of the flow has recently been observed experimentally by the MSU $4 \pi$ group (Krofcheck et al 1989, 1992, Ogilvie et al 1990, Wilson et al 1990, Westfall et al 1993) and at the GANIL facility (Sullivan et al 1990).

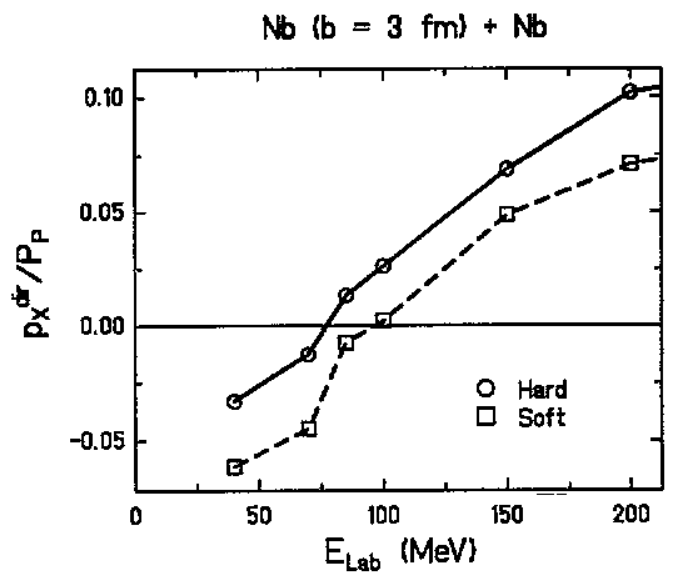

Figure 25. The relative transverse momenta $p_{x} / p_{\text {beam }}$ are shown for the reaction $\mathrm{Nb}(b=3 \mathrm{fm})+\mathrm{Nb}$ obtained with the vul model using a soft and a hard EOS (Hartnack 1992).

In figure 25 we present a vuU calculation which shows this onset of the flow for different interactions. It can be seen that the crossing point, where the transverse momentum transfer changes its sign, depends clearly on the EOS. A stiffer EOS leads to higher compression energies which result in a lower energy for the crossing point. Note, however, that these calculations were performed at the fixed impact parameter $b=3 \mathrm{fm}$. Since the transverse momentum transfer also depends strongly on the impact parameter and this impact parameter cannot be measured with sufficient precision at these energies, it seems to be almost impossible to use this effect in order to pin down some information about the EOS. A more detailed comparison of the recent MSU data with the BUU model calculations has recently been made by Westfall et al (1993). They showed that the in-medium nucleonnucleon scattering cross sections have an even larger influence on the onset of the flow than the nuclear EOS.

4.1.2. The $90^{\circ}$ squeeze-out. Let us now concentrate on the behaviour of the particles that are stopped at midrapidity. These particles are supposed to undergo the highest compression and are therefore expected to be most sensitive to the underlying EOS. Unfortunately the usual construction of the bounce-off observables triggers on particles which are not at midrapidity but at forward/backward rapidities. For this reason we now study the so-called squeeze-out, namely the component of the collective flow that is emitted perpendicular to the reaction plane. 
For the qualitative and quantitative comparison with the latest Plastic Ball data we therefore look at the azimuthal distribution of the particles at midrapidity. The azimuthal angle $\varphi$ is defined as the inclination angle between the $p_{\perp}$-vector and the $x$-axis. A $\varphi$ angle of $0^{\circ}$ corresponds to particles in the positive hemisphere of the reaction plane (projectile spectators) and $180^{\circ}$ to particles in the negative hemisphere of the reaction plane (target spectators). In $90^{\circ}$ and $270^{\circ}$ one observes the particles with momenta perpendicular to the reaction plane.

The first squeeze-out studies, done by the Plastic Ball collaboration (Gutbrod et al $1989 \mathrm{~b}$ ) employed an analysis that used the transverse momentum analysis to determine the reaction plane and the sphericity method to determine the flow angle in that reaction plane. These investigations have demonstrated that the azimuthal distributions around the flow axis are much better suited to describing the event than the azimuthal distribution around the beam axis! It was also shown that the azimuthal distribution of the momentum per nucleon of the particles also shows the squeeze-out effect, indicating that not only the density of particles is enhanced in the out-of-plane direction, but that these particles are also emitted with a higher average transverse momentum.
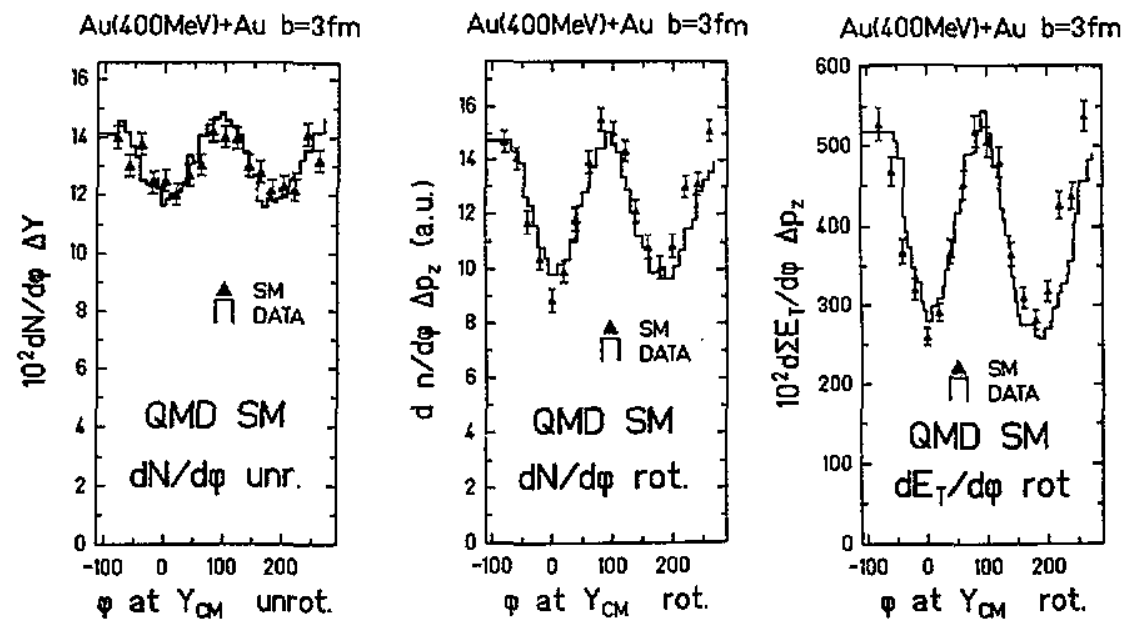

Figure 26. Azimuthal distributions for the reaction Au $(400 \mathrm{~A} \mathrm{MeV})+\mathrm{Au}$. The Plastic Bal] data (full triangles) are compared with the vuU results (histogram) obtained with a soft Eos plus MD! (taken from Hartnack (1992)).

In figure 26 we compare the azimuthal distributions $\mathrm{d}^{2} N / \mathrm{d} \varphi \mathrm{d} y$ in the unrotated (lefthand figure) and the rotated (middle figure) frame as well as the azimuthal distribution of the transverse energy $\mathrm{d}^{2} E_{\mathrm{T}} / \mathrm{d} \varphi \mathrm{d} y$ (right-hand figure) with the Plastic Ball data (Gutbrod et al 1989b). The comparison is made for the reaction $\mathrm{Au}(400 \mathrm{AMeV})+\mathrm{Au}$ at the corresponding impact parameter respectively multiplicity. The best agreement between the QMD calculations (full triangles) and the data (histogram) is found when the soft EOS plus MDI is used. One should, however, keep in mind that the calculations were for the fixed impact parameter $b=3 \mathrm{fm}$, while the data were selected according to the charge particle multiplicity.

Further experimental (Gutbrod et al 1990b) and theoretical (Hartnack 1992) investigations have shown that the squeeze-out effect is a truly collective effect that scales linearly 
with the mass of the system rather than with $\sqrt{A}$ like the bounce-off. The maximum of the squeeze is found at surprisingly low beam energies $(E \approx 400 \mathrm{AMeV})$, in contrast to the bounce that increases up to the highest energies that have been achieved up to now.

Because the squeeze-out particles escape directly from the hot and dense reaction zone, unhindered by the surrounding cold target or projectile matter, they provide a clear probe through which one can look directly at the compressed and hot fireball. The simultaneous description of both collective flow effects within one microscopic approach is therefore a large step forward to the ultimate goal of heavy-ion physics, namely the determination of the bulk properties of nuclear matter.

\subsection{Stopping and thermalization}

Before we investigate the degree of stopping and thermalization we start with a survey of the time evolution for the reactions $\mathrm{Au}(200,800 \mathrm{AMeV}, b=0 \mathrm{fm})+\mathrm{Au}$. In order to distinguish between different groups of nucleons we define, in addition to projectile and target nucleons, a third component that includes all nucleons that have suffered at least one collision.

A.s $\{200 \mathrm{MeV} /$ nucl., $\mathrm{b}=0 \mathrm{fm}\}+\mathrm{Aus}$

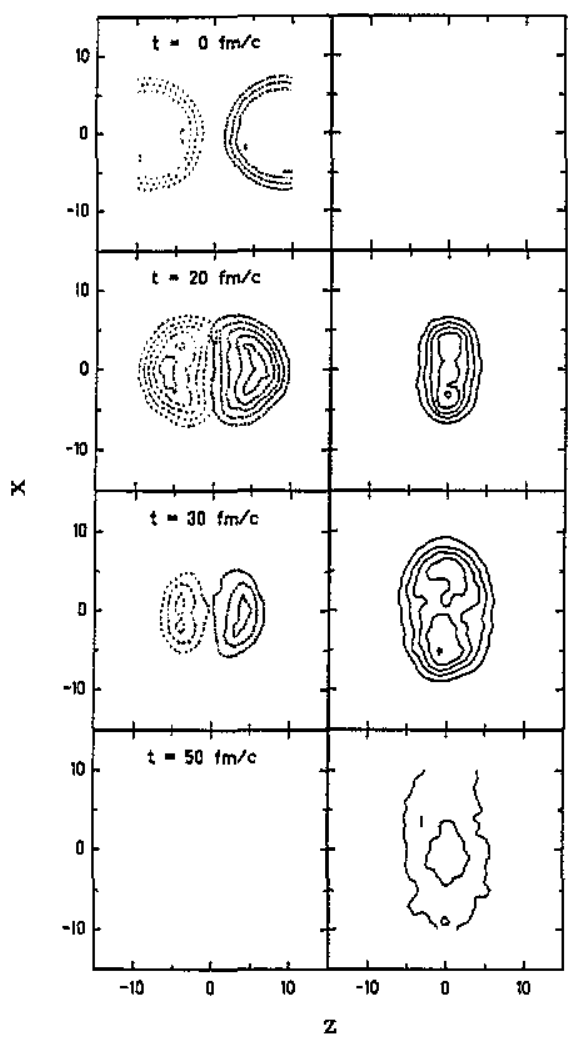

Figure 27. Time evolution of the density contours of the system ${ }^{197} \mathrm{Au}(200 \mathrm{~A} \mathrm{MeV}, b=0 \mathrm{fm})+{ }^{197} \mathrm{Au}$ for target and projectile nucleons which have not yet collided (left-hand side) and for the 'participant' component (right-hand side), which includes all particles which have collided at least once. Note that the spectator fluids barely interpenetrate, but rather collide with the participant nucleons piled up in an ellipsoid at midrapidity (Berenguer et al 1992).

Figure 27 shows in the left-hand column the density profiles of the (cold) projectile and target component, i.e. all particles that have not collided up to this time. The right-hand column shows the corresponding profiles of the participant component. Only at the very beginning of the reaction is there an overlap between the projectile and target component. 
The very first collisions rapidly built up the participant component, which immediately after formation acts as a buffer between target and projectile. Hence they no longer interact directly. The further evolution of the system is completely determined by the separate interaction of the participant matter with the projectile and target, respectively. Interactions within the participant matter are crucial, too. This behaviour is in complete contradiction with the two-fluid model (Amsden et al 1977b, Ivanow et al 1985, Clare and Strottman 1986, Mishustin et al 1989, Satarov 1990), where one assumes that the projectile and target component stream through each other and collectively decelerate.

Let us now investigate the influence of the collisions as well quantum effects such as Pauli blocking and the possible reduction in the nucleon-nucleon cross section on the reaction. This influence can most clearly be seen by inspection of the mean free path of the nucleons.

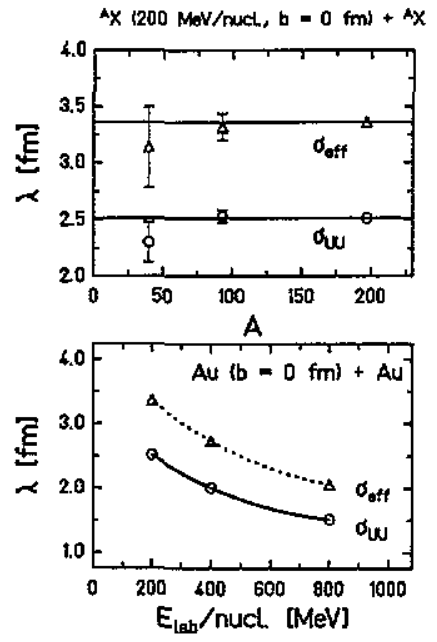

Figure 28. The mean free path $\lambda$ of the nucleons is shown for the very central collisions at energies from 200 to $800 \mathrm{AMeV}$ for the different $\mathrm{N}-\mathrm{N}$ cross sections, masses and energies as indicated (Berenguer er al 1992). Note that $\lambda$ does not depend on the mass of the system and decreases with increasing bombarding energy in contrast to the naive expectation of classical kinetic theories (Sobel et al 1975).

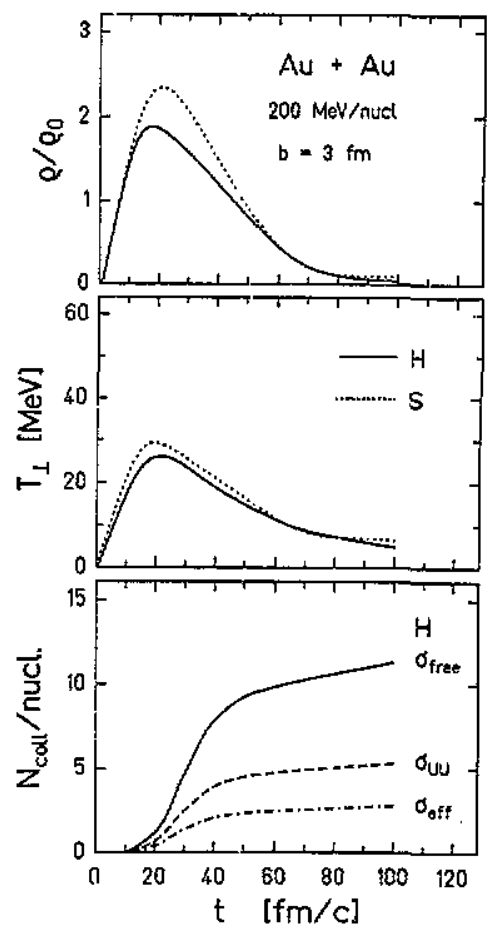

Figure 29. Time evolution of the central compression, the transverse 'temperature' and the average number of collisions per nucleon of the renction ${ }^{197} \mathrm{Au}$ (200A MeV, $b=3 \mathrm{fm})+{ }^{197} \mathrm{Au}$. The local 'temperature' has been obtained via the relation $T=$ $\left\langle p_{\perp}^{2}\right) / 2 m$ for the different interactions as indicated. Almost afl collisions take place when the 'temperature' and densities are at their highest values (Berenguer et al 1992).

Figure 28 (upper part) shows the dependence of the mean free path on the mass of the system for central, symmetric reactions. The results are shown for the UU cross section $\sigma^{\mathrm{UU}}$ and for the $30 \%$ reduced cross section $\sigma^{\text {eff }}$. This reduction in the cross section clearly reduces the number of collisions and therefore increases $\lambda$. Note that $\lambda$ depends on the 
$\mathrm{N}-\mathrm{N}$ cross section and not on the mass of the system. The energy dependence of $\lambda$ can be studied for the most central collisions of $\mathrm{Au}$ on $\mathrm{Au}$ in the lower part of figure 28. $\lambda$ decreases with increasing bombarding energy, due to the higher densities achieved, and the lower Pauli blocking rates (cf figure 10) in spite of a decreasing $\mathrm{N}-\mathrm{N}$ cross section. The results obtained with the Boltzmann cross section $\sigma^{\text {free }}$ (without Pauli blocking) are also shown. This increased cross section yields a drastic decrease in the mean free path. Enhanced thermalization and nuclear stopping results.

Let us now inspect in more detail the stopping of the incoming matter which implies the equilibration of the incident longitudinal momenta in $\mathrm{N}-\mathrm{N}$ collisions. Some quantities of interest to macroscopic models are shown in figure 29 for the reaction $\mathrm{Au}(200 \mathrm{~A} \mathrm{MeV}$, $b=3 \mathrm{fm}$ ) + Au as a function of time. The central density increases from zero (in the initial stage the nuclei are separated in coordinate space) to the maximum density reached after $\sim 15 \mathrm{fm} c^{-1}$. The maximum densities achieved in this energy regime are between two and three times higher than the normal nuclear matter density depending on the EOS used (with a soft EOS higher densities are reached). After this compression stage the matter flows out of the central region and the central density decreases steadily to zero. The same behaviour can be observed in the middle part of figure 29 for the transverse kinetic 'temperature', which is defined as $T_{\perp}=\left(\left\langle p_{x}^{2}\right\rangle+\left\langle p_{y}^{2}\right\rangle\right) /(2 m)$.

The reason for the degradation of the longitudinal momenta into transverse degrees of freedom, the build up of the compression zone and the complete stopping of the system are the hard nucleon-nucleon collisions. The lower part of figure 29 shows therefore the average number of $\mathrm{N}-\mathrm{N}$ collisions per nucleon plotted as a function of time for the different $\mathrm{N}-\mathrm{N}$ cross sections. Almost all collisions take place in the time interval when the 'temperature' and the density are at their highest values. At the end of the compression stage $(t \approx 50$ $60 \mathrm{fm} c^{-1}$ ), practically all collisions have ceased. Hence, the following expansion and fragmentation stage is little affected by short-range interactions and the system evolves almost isentropically, although there are still a few collisions occurring within the formed fragments.

The large average collision numbers $N_{\text {coll }} / A \approx 5$ indicate the approach to local equilibrium, since kinetic models predict the thermalization of the incident momenta already after two or three collisions, depending on the beam energy (Randrup 1979, Cugnon et al 1981, Rosenhauer et al 1984).

The dependence of the $\mathrm{d} N / \mathrm{d} Y$ distributions on the mass of the system and on the bombarding energy is shown in the upper part of figure 30: complete stopping, resulting in a single-peak distribution at midrapidity is observed only for massive systems while lighter systems exhibit rather broad rapidity distributions even for very central collisions. The stopping power does not change much with bombarding energy, if the scaled rapidity distribution $\mathrm{d} N / \mathrm{d}\left(Y / Y_{\mathrm{P}}\right)$ is considered, as can be seen in the lower part of figure 30 .

Figure 31 shows the QMD calculations (right-hand column) compared with the Plastic Ball data (Gutbrod et al 1990a) (left-hand column) for different systems at $400 \mathrm{~A} \mathrm{MeV}$ bombarding energy. The shape of the distributions seem to be identical for all systems. However, this insensitivity is only due to the selection of protons (light clusters are predominantly seen at the projectile and target rapidities (Peilert et al 1988)). For the smaller systems there is more transparency, because the ratio of the mean free path to the diameter of the system is not small enough. The Plastic Ball efficiencies which have been applied to the theoretical results furthermore cut out those particles, which remain at target and projectile rapidities, because of their low energy, respectively small angles, in the laboratory frame. Thus the plateau shapes of the unfiltered distributions as can be seen in figure 30 appear as Gaussian shaped only due to the efficiency cuts. Non-negligible 

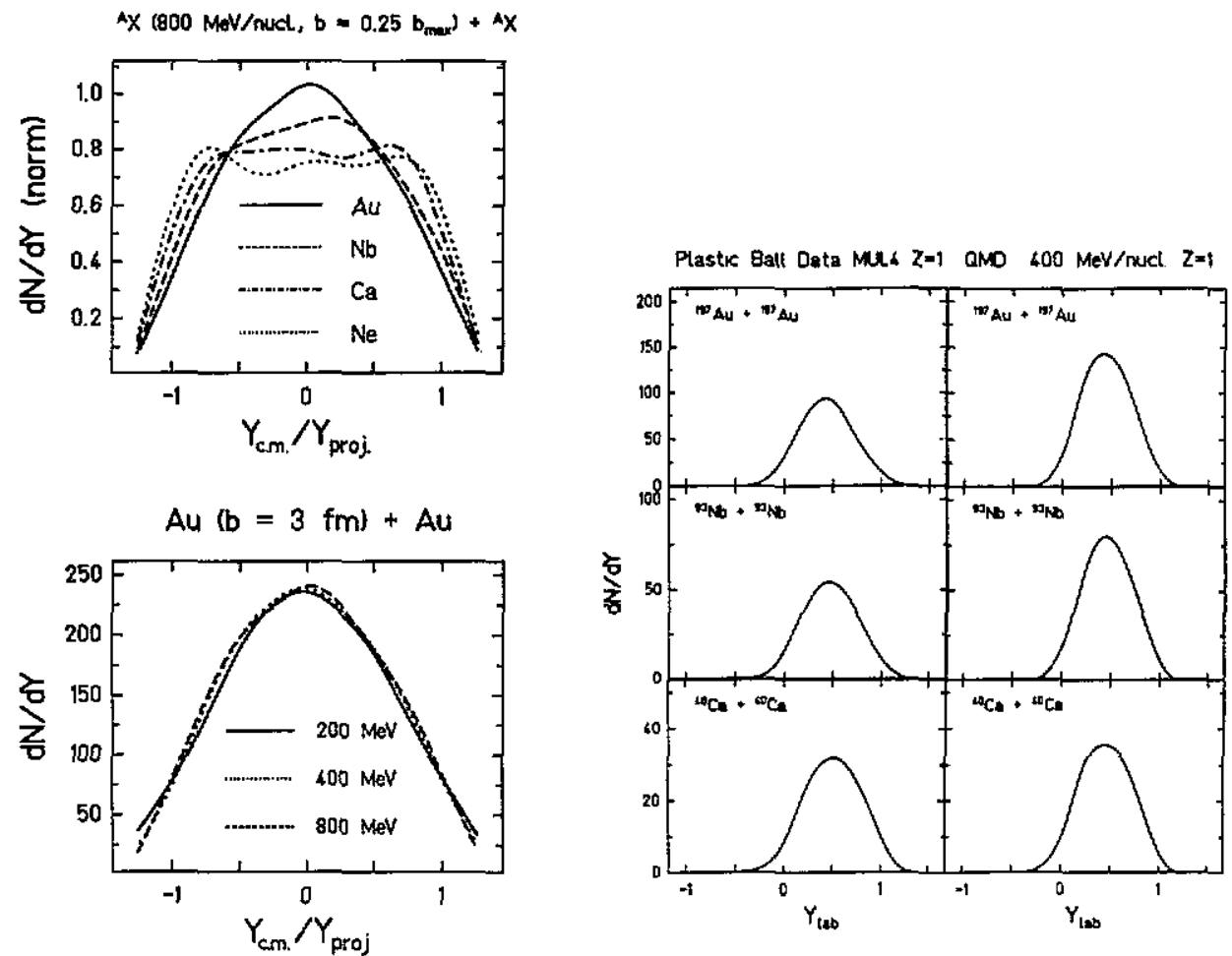

Figure 30. Mass (upper figure) and energy (lower figure) dependence of the rapidity distributions for the indicated systems. Complete stopping is only observed for massive systems at all energies investigated (Berenguer et al 1992).

Figure 31. Mass dependence of the proton rapidity distributions. The QMD results (right-hand column) are compared to the Plastic Ball data (Gutbrod et al 1990a) (left-hand column). Due to the efficiency cuts of the Plastic Ball filter all distributions (even those for the light systems) look Gaussian shaped (Berenguer et al 1992).

distortions of the midrapidity yields have been reported for the very heavy system by the Plastic Ball collaboration.

The sensitivity of nuclear stopping to the potential employed and to the effective scattering cross section has also been investigated. Figure 32 shows that the influence of the mean field on the longitudinal flow is relatively small. However, the scattering cross section is vital for the stopping power. By using the free cross section (without Pauli blocking) the classical collision numbers double. This yields an increase in the stopping power. A global reduction in the scattering cross section with $\sigma^{\text {eff }}=0.7 \sigma^{\mathrm{UU}}$ in combination with the soft EOS and the MDI (SIM) yields double-peaked $\mathrm{d} N / \mathrm{d} Y$ distributions with peaks closer to the rapidities of the projectile and target. The double-peak structure originates from. the remnants of the projectile and target spectators. It indicates an incomplete stopping of the incident nuclei. One should, however, keep in mind that a more complex functional dependence of $\sigma^{\text {eff }}$, e.g. on $\rho$ and $\mathrm{T}$, could render the systematics of the interplay between the effective scattering cross section and the longitudinal and transverse flow much more complicated. The $\mathrm{d} N / \mathrm{d} Y$ distribution of the soft EOS with the UU cross section $\sigma^{\mathrm{UU}}(S)$ lies between the curves $\mathrm{S}$ and $\mathrm{SLM}$. The $\mathrm{MDI}$ (SM) modifies the stopping power slightly. The evident differences between the $\mathrm{d} N / \mathrm{d} Y$ spectra obtained from the cases $\sigma^{\text {eff }}$ and $\sigma^{\mathrm{uU}}$ can be 
$\mathrm{Au}(200 \mathrm{MeV} /$ nucl., $\mathrm{b}=3 \mathrm{fm})+\mathrm{Au}$

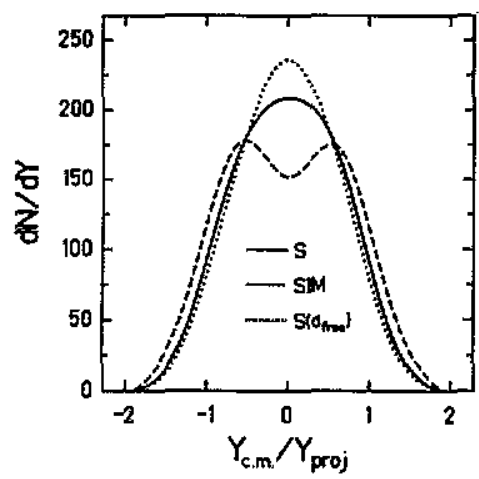

Figure 32. The influence of the different interactions on the rapidity distributions $\mathrm{d} N / \mathrm{d} Y$ (all particles included) obtained from the reaction ${ }^{197} \mathrm{Au}(200 \mathrm{AMeV}, b=$ $3 \mathrm{fm})+{ }^{197} \mathrm{Au}$ (Berenguer et al 1992). The $\mathrm{d} N / \mathrm{d} Y$ distributions depend strongly on the $\mathrm{N}-\mathrm{N}$ cross sections used!
Au $(b=0 \mathrm{fm})+A u$

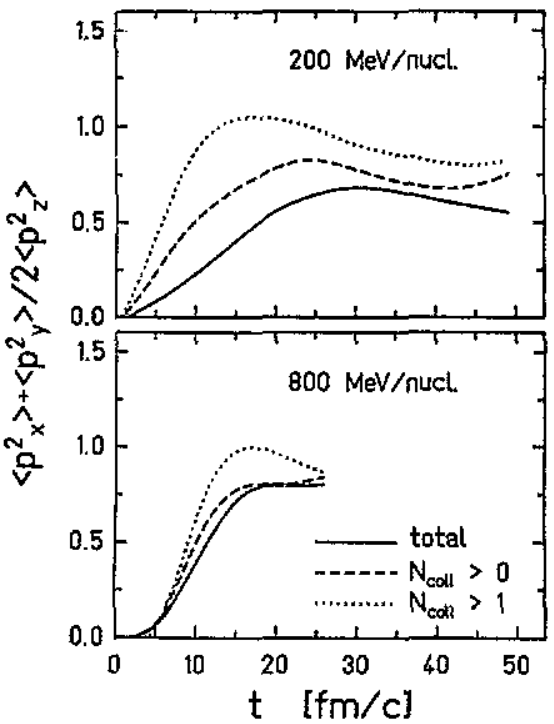

Figure 33. Ratio of the mean quadratic momenta at the origin of the $\mathrm{cM}$ frame in transverse and longitudinal direction for centrol $\mathrm{Au}+\mathrm{Au}$ collisions at $200 \mathrm{~A} \mathrm{MeV}$ (upper figure) and $800 \mathrm{AMeV}$ (lower figure) for all particles (full curves), for particles which have collide at least once (broken curves) and more than once (dotted curves). The participant component equilibrates much better than the spectators. This behaviour is more pronounced at lower energies (Berenguer et al 1992).

exploited for the experimental determination of $\sigma^{\text {eff }}$ from the $\mathrm{d} N / \mathrm{d} Y$ spectra for different systems at different energies (see, e.g., Peilert et al 1988, Keane et al 1988). The measured rapidity spectra for the systems $\mathrm{Ar}(1200 \mathrm{~A} \mathrm{MeV})+\mathrm{BaI}_{2}$ and $\mathrm{KCl}$ are in good agreement with vUU calculations (Keane et al 1988), which use the free scattering cross section $\sigma^{\text {UU }}$. A reduced-or increased- $\sigma^{\text {eff }}$ fails to reproduce these data, just as the $\mathrm{Au}+\mathrm{Au}$ data show no dip.

Let us now investigate to what extent thermodynamic concepts, in particular local equilibrium, are justified. The local equilibrium concept forms the basis of ideal fluid dynamics while the assumption of global equilibrium forms the basis of most statistical models.

The local degree of isotropy $R$ can be defined as $R=\left\langle p_{T}^{2}\right\rangle / 2 \cdot\left\langle p_{\mathrm{L}}^{2}\right\rangle$, where $p_{\mathrm{T}}$ and $p_{L}$ are taken in the rest frame of the matter element under consideration. Then $R \approx 0$ means total anisotropy which is characteristic for the first stage of a collision in which the two nuclei just touch and no transverse momentum yet has been transferred. An isotropic momentum distribution would lead to $R=1$. Note, however, that $R=1$ is a necessary, but not sufficient condition for equilibration. For heavy systems one expects to be close to a local equilibrium situation, where viscous hydrodynamics is applicable. When the matter starts to decompress, near equilibration is observed in this central zone. Figure 33 shows the time evolution of $R$ at the origin ( $x=y=z=0$ ) for the reaction Au (200, $800 \mathrm{~A} \mathrm{MeV}, b=0 \mathrm{fm}$ ) $+\mathrm{Au}$. Three different cases are shown, including all nucleons (full curve), those nucleons which have suffered at least one (broken curve) or even two (dotted 
$A u(b=0 \mathrm{fm})+A u$

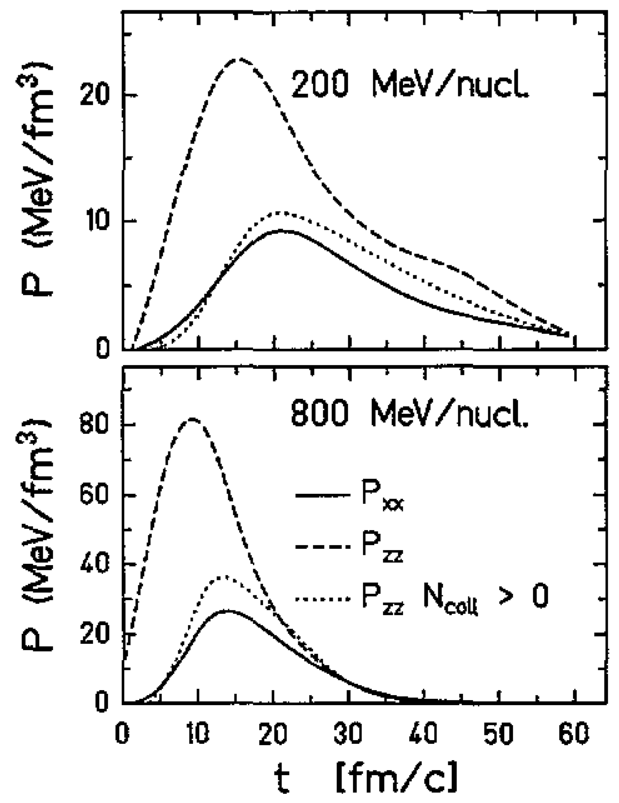

Figure 34. Time dependence of the kinetic pressure in the centre of the reaction for the system Au (200 (upper figure), 800 (lower figure) $A \mathrm{MeV}, b=0 \mathrm{fm}$ ) $+A$. The broken curves correspond to the $x x$ and yy components of the stress tensor, full curves to the $z z$ component. A thermal (chain curves) and a nonthermal part (full curves) is defined for nucleons which have suffered at least one collision (Berenguer et al 1992).

curve) collisions. Local equilibrium is clearly not achieved for the total system while the participant component equilibrates better $(R \approx 0.8-0.9)$.

More information about the thermalization process can be obtained from the stress tensor

$$
P_{k l}(\boldsymbol{r}, t)=\int \mathrm{d}^{3} p f(\boldsymbol{p}, \boldsymbol{r}, t)\left[p_{k}-\left\langle p_{k}(r, t)\right\}\right]\left[v_{l}-\left\langle v_{l}(r, t)\right\rangle\right] \quad k, l=x, y, z .
$$

The interaction part of $P_{i k}$ has been excluded from the analysis, because $P_{i k}$ is to be compared with the Newtonian ansatz used in viscous fluid dynamics (Csernai and Barz 1981, Buchwald et al 1981, 1983a). For the local interactions used here $P_{i k}$ is trivially isotropic. This means the sum of the kinetic and interaction pressure will always appear more isotropic than the kinetic pressure (stress tensor) alone. Using the QMD distribution function (see equations (31), (32)) one obtains

$p_{k l}(r, t)=\frac{1}{(2 \pi L)^{3 / 2}} \sum_{i=1}^{N} \exp \left\{-\frac{\left(r-r_{i 0}(t)\right)^{2}}{2 L}\right\}\left[p_{i 0}-\langle p(r, t)\rangle\right]_{k}\left[\left(p_{i 0} / m_{i}\right)-\langle v(r, t)\}\right]_{l}$

Figure 34 shows the time evolution of the different components of the stress tensor. The maxima of both the $z z$ component (full curve) and the $x x$ component (broken curve) appear at the same time as the maxima of the temperatures and the central densities (cf figure 29). However, the absolute values of these components differ from each other; they approach one common value towards the end of the reaction. This reflects again the importance of treating the non-equilibrium aspects during the early course of the reaction. On the other hand, for fireball nucleons the $z z$ component of the stress tensor (dotted curve) is almost identical to the $x x$ component, i.e. the fireball is near thermal equilibrium. Hence, the basic assumption of the three-fluid model (Csernai et al 1982, Rosenhauer et al 1987) is justified: each of the three components closely approach thermal equilibrium. The non-equilibrium effects are most important at the start of the reaction. 
Can the concept of viscous fluid dynamics (Csernai and Barz 1981, Buchwald et al 1981, 1983a) be applied to heavy-ion collisions? The viscosity coefficients $\eta$ (shear viscosity) and $\xi$ (bulk viscosity) are defined by the following Newtonian form of the stress tensor.

$$
P_{i j}=p \cdot \delta_{i j}-\eta \cdot\left(\frac{\partial V_{i}}{\partial x_{j}}+\frac{\partial V_{j}}{\partial x_{i}}-\delta_{i j} 2 / 3 \cdot \sum_{k=1}^{3} \frac{\partial V_{k}}{\partial x_{k}}\right)-\delta_{i j} \cdot \zeta \cdot \sum_{k=1}^{3} \frac{\partial V_{k}}{\partial x_{k}}
$$

The viscosity and its density and temperature dependence serve as a constitutive equation for hydrodynamical calculations. In a microscopic model these viscosity coefficients can be determined by comparing the exact pressure tensor (equation (48)) with the Newtonian form (equation (50)). Furthermore this Newtonian ansatz itself can be checked. This means that unique coefficients $\eta$ and $\xi$ should be found so that all, in general, anisotropic components of the stress tensor obey relation (50) with the same coefficients. However, we find here that the coefficients for the longitudinal components of the stress tensor are about a factor of three larger than the coefficients for the transverse component.

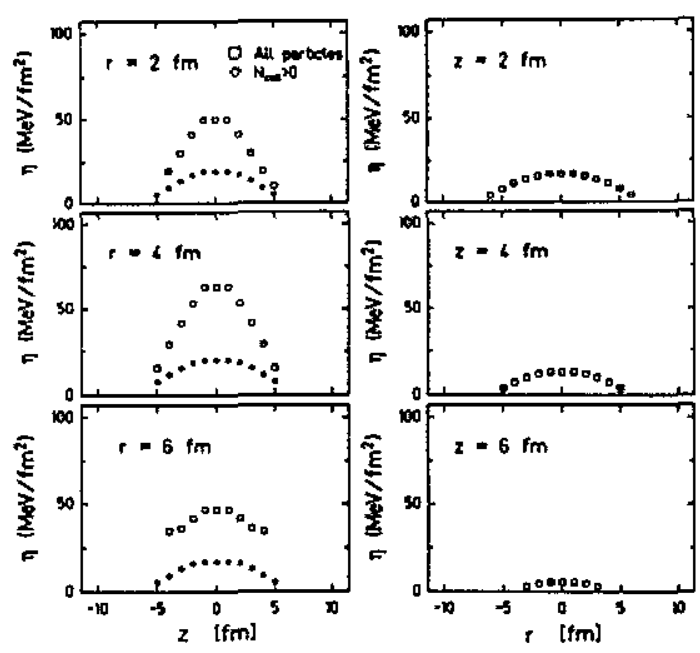

Figure 35. Shear viscosity coefficient $\eta$ extracted from QMD calculations in the reaction Au $(200 \mathrm{~A} \mathrm{MeV}, b=0 \mathrm{fm})+\mathrm{Au}$ at $t=$ $20 \mathrm{fm} \mathrm{c}^{-1}$ along the beam direction $z$ (left-hand side) and in transversal direction $r$ (right-hand side). Note the apparent difference between the viscosity coefficient in the longitudinal and transverse directions (Berenguer et al 1992).

Figure 35 shows the shear viscosity coefficient as a function of both the transverse (denoted with $r$ ), and the longitudinal (denoted with $z$ ) distance from the origin, extracted for the reaction $\mathrm{Au}(200 \mathrm{~A} \mathrm{MeV}, b=0 \mathrm{fm})+\mathrm{Au}$, at $20 \mathrm{fm} c^{-1}$ (note the cylinder symmetry of the system). The left-hand column shows the longitudinal viscosity coefficient $\eta_{r z}$ for all particles and for particles which have collide at least once. $\eta_{r z}$ reaches maximum values around $60 \mathrm{MeV} \mathrm{fm} \mathrm{m}^{-2} \mathrm{c}^{-1}$ in the highest density region, if all particles are considered. It drops to zero at larger distances from the centre due to the density and temperature dependence. In contrast, the participant component reaches values of $\approx 20 \mathrm{MeV} \mathrm{fm}{ }^{-2} c^{-1}$ only. The right-hand column shows the transverse component $\eta_{x y}$, which reaches maximum values of $20 \mathrm{MeV} \mathrm{fm}^{-2} c^{-1}$, which are nearly identical to the 'participant' component. The same situation is found for higher energies (Berenguer et al 1992). A maximum viscosity coefficient of $130 \mathrm{MeV} \mathrm{fm}^{-2} c^{-1}$ is needed in order to get a proportionality between stress tensor and velocity gradient at $800 \mathrm{~A} \mathrm{MeV}$ bombarding energy. For the transverse component a maximum value of $20 \mathrm{MeV} / \mathrm{fm}^{2} c$ is found. A higher value of the viscosity coefficient is found in the longitudinal direction. 
Previous one- (Csernai and Barz 1981), two- (Buchwald et al 1981) and threedimensional (Buchwald et al 83a) viscous hydrodynamical calculations used much smaller viscosity coefficients than those obtained by kinetic theories. These low values were motivated by fission fragment spectra (Hofmann and $\mathrm{Nix}$ 1983). However, recently hot fission experiments recently led to much higher extracted viscosity coefficients (Paul Private communication) in accordance with the estimates of kinetic theories (Bodmer 1978, Danielewicz 1984). Recent hydrodynamical calculations (Schmidt 1989) show that only such high viscosity coefficients can explain the flow and bounce-off data. However, viscosity coefficients of $60 \mathrm{MeV} \mathrm{fm}{ }^{-2} c^{-1}$ yield better agreement with the experimentally observed in-plane sidewards while the out-of-plane squeeze-out can be reproduced with $\eta=40 \mathrm{MeV} \mathrm{fm}^{-2} c^{-1}$ (see also (Schürmann 1988)). This is in qualitative agreement with the above results.

\section{Multifragmentation}

One goal of heavy-ion physics is to investigate nuclei at the extremes of excitation. In the decay of highly excited nuclei it is possible to study the low density part of the nuclear EOS, since at those densities $\left(\varrho<\varrho_{0}\right)$ the behaviour of the system is governed by the balance of long-range attractive and short-range repulsive nuclear forces with the long-range Coulomb force. In analogy with the classical Van der Waals gas, where the interplay between longrange attractive and short-range repulsive forces leads to a second-order phase transition, such a liquid-vapour phase transition is also expected in heavy-ion collisions (Friedman and Pandharipande 1981, Bertsch and Siemens 1983, Siemens 1983, Goodman et al 1984, Panagioutou et al 1984, Csernai and Kapusta 1986, Hahn and Stöcker 1988). In low-energy heavy-ion collisions or high-energy proton-induced reactions this phase transition results in an increase in the total entropy (Csernai and Kapusta 1986) and in the fragmentation of the system (Fisher 1967). While the entropy is not directly measurable in heavy-ion experiments, the formation of intermediate mass fragments (IMFs) can be observed directly. This field was first touched upon experimentally via inclusive proton-induced reactions (Finn et al 1982) and later in heavy ion reactions (Aleklett et al 1982, Jakobsson et al 1982, Jacak et al 1983, 1987, Warwick et al 1983, Waddington and Freier 1985, Doss et al 1986, Gelbke and Boal 1987, Alard et al 1987, Trockel et al 1987, 1988, 1989, Pochodzalla 1989, Ogilvie et al 1989, 1990, Aleklett et al 1990, Bowman et al 1991).

All the inclusive, i.e. impact parameter averaged, experiments have shown a typical power-law dependence $\sigma(A) \approx A^{-\tau}$ of the mass yield distributions, where the critical exponent $\tau$ was compatible with a value of approximately 2.5 . Fisher (1967) made such a prediction for fragmentations resulting from a classical liquid-vapour phase transition only at the critical point.

The major shortcoming of these experiments was, however, that no selection on multiplicity (impact parameter) could be done, and so the results could also be of geometrical or any other origin and not a signature of a phase transition.

In recent years it has become possible to do exclusive measurements of multifragmentation reactions. This has been done with streamer chamber detectors (Jakobsson et al 1982, Waddington and Freier 1985) or with electronic detectors (Doss et al 1986, Alard et al 1982, 1987, Ogilvie et al 1989, 1991, Hubele et al 1991, Bowman et al 1991, Sangster et al 1992, Hjort et al 1993). With the availability of $4 \pi$ detectors it is possible to study the multifragment break up of nuclear systems in much greater detail, due to the huge number of events which can be sampled. 
The decay of highly excited nuclei may be divided up qualitatively into two processes; a dynamically determined fast process in which many nucleons and some clusters escape the equilibrating mix and a statistical process that governs the decay of the equilibrated residue. The consequences of the dynamic processes in heavy-ion collisions have been discussed in the first sections of this article. Now we want to concentrate on the process of fragment formation. In the past few years much progress has been made in describing the dynamical stage of these reactions, but the statistical processes have had less attention. So let us first summarize the properties of common statistical decay models.

At moderate excitations (for temperatures up to a few $\mathrm{MeV}$ ) the decay of equilibrated nuclei may be interpreted in terms of sequential binary decay. Straightforward extensions of the Weisskopf and Ewing (1940) evaporation formula have been used in this context, with moderate success in reproducing spectra and yields of ejectiles, including clusters, and exclusive cluster multiplicities. These approaches rely on phase space, but restrict the phase space at each step of the calculation to that available for binary processes.

As the excitation energy increases, three-body and higher order (simultaneous) decay processes will show a greater phase space than binary processes. Different physical models (formulations) are necessary to consider these higher order processes. The question as to whether the greater phase space will determine the outcome of these reactions or whether dynamic constraints (as for example seen in nuclear fission) will restrict the multifragmentation channels remains to be seen as a result of experimental measurement.

The decay models treat this process in a statistical way in micro canonical, canonical or grand canonical ensembles (Randrup and Koonin 1981, Fai and Randrup 1982, 1983 , Gross and Zhang 1985, Bondorf et al 1985a, b, Gross et al 1986, Koonin and Randrup 1987, Botvina et al 1987, 1990, Hahn and Stöcker 1988, Gross 1990); or by means of evaporation models (Blann 1985, Barbagallo et al 1986, Friedman 1988, Blann et al 1989, 1991). All these models have the great disadvantage that they start with an equilibrated system. While this may be a good approximation in heavy-ion reactions at very low energies (around the Coulomb barrier the projectile may excite the collective degrees of freedom of the target) or for light-ion-induced reactions at very high energies, it is still an open question whether at higher energies a global, or even a local, equilibrium is achieved (see also the discussion in the previous section).

The aim of the present review is to present a realistic model, capable of describing the whole collision process, starting from the fast initial stage of interacting projectile and target and ending with the final stage with the de-excitation of thermalized intermediate states. It seems that a practical way to solve this problem will be to combine several models, each suitable for the description of a limited part of the whole dynamical process.

In the mean-field models of vUU/BUU type the nucleons follow a trajectory which is generated by the ensemble averaged motion of all the other nucleons (cf section 3.2). This means that two-body and higher-order correlations are ignored, and these models give only event-averaged answers for many-body observables such as the formation of fragments (Kruse 1985a, Koch et al 1991).

The only microscopic model which is able to describe the direct fragment formation is the QMD model, which was described in detail in section 3.2.2 of this article.

First we want to study some general multifragmentation properties calculated with the original QMD model (without the Pauli potential). The spatial evolution of a $\mathrm{Au}+\mathrm{Au}$ collision at $200 \mathrm{~A} \mathrm{MeV}$ energy can be followed in figure 36 for impact parameters 1,3 and $7 \mathrm{fm}$. The beam axis coincides with the $z$ direction. Observe the formation of one blob of matter for all impact parameters $\left(t \leqslant 40 \mathrm{fm} c^{-1}\right.$ ), which then disintegrates and yields the fragments. For half-overlap collisions $(b=7 \mathrm{fm})$ two massive projectile and target 
remnants survive the reaction. In this case one observes almost no IMFs. When going to more central collisions the projectile and target-like fragments become smaller $(b=3 \mathrm{fm})$ and are absent in the most central collisions, where we observe a complete disintegration of the incident nuclei. On the other hand, the IMFs are abundantly produced in central collisions and disappear for peripheral ones.

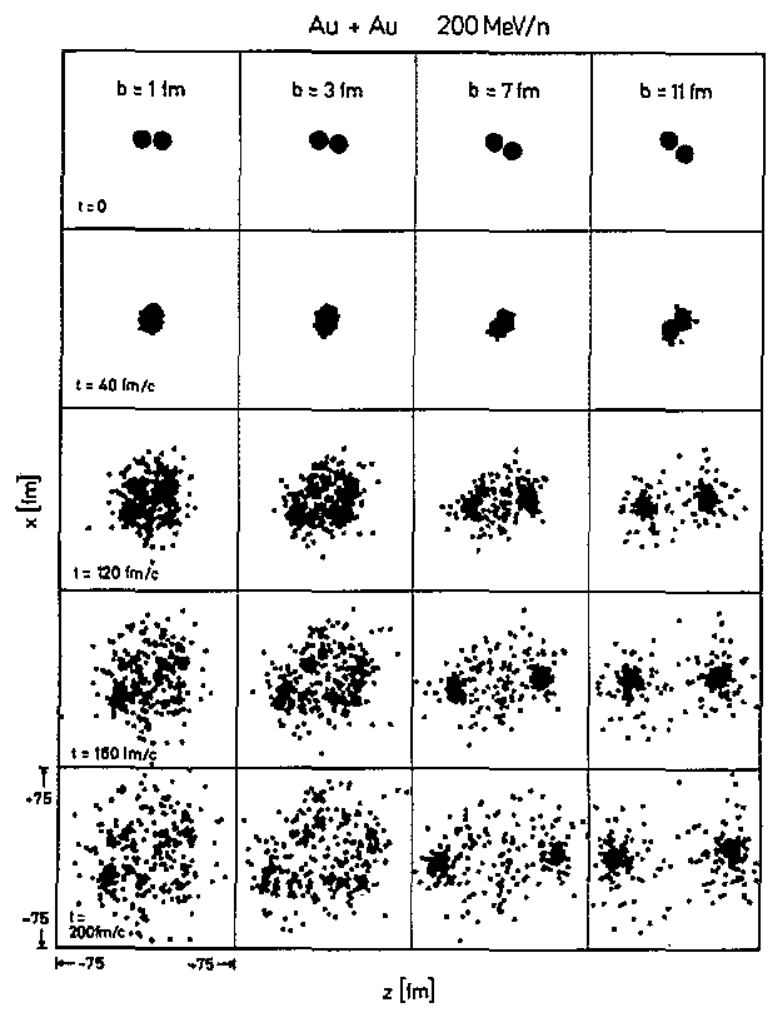

Figure 36. Time evolution of the particle distribution in configuration space for the reaction $\mathrm{Au}(200 \mathrm{~A} \mathrm{MeV}, b=1,3,7$ and $11 \mathrm{fm})+\mathrm{Au}$. The projection of all particles onto the reaction plane is displayed at five different times as indicated.

In figure 37 we show the typical time-structure of a single half-overlap collision for $\mathrm{Au}(200 \mathrm{~A} \mathrm{MeV}, b=7 \mathrm{fm})+\mathrm{Au}$ in more detail. Only fragments with $A \geqslant 10$ are considered from $t=0$ (bottom) to $t=200 \mathrm{fm} c^{-1}$ (top) in steps of $10 \mathrm{fm} c^{-1}$. Up to $50 \mathrm{fm} c^{-1}$ one observes one blob of matter in configuration space which-for this large impact parameter-is still separated in momentum space into a projectile and a target-like residue. After $50 \mathrm{fm} c^{-1}$ the system breaks up into these two residues. Between 30 and $80 \mathrm{fm} c^{-1}$ most of the single nucleons and light fragments $(A<10)$ are evaporated. Then a rather stable fragment with $A \approx 105$ remains in the projectile rapidity regime. In the target regime a second break up is observed, which yields two stable fragments with $A \approx 80$ and 15 , respectively. At large impact parameters $(b=7 \mathrm{fm})$ the IMFs are mainly produced in the binary break up of the heavy residues.

Figure 38 presents the average number of fragments as a function of time for $b=3 \mathrm{fm}$. First of all we observe that the mass yield distribution for all fragments $(A \geqslant 2)$ stabilizes 


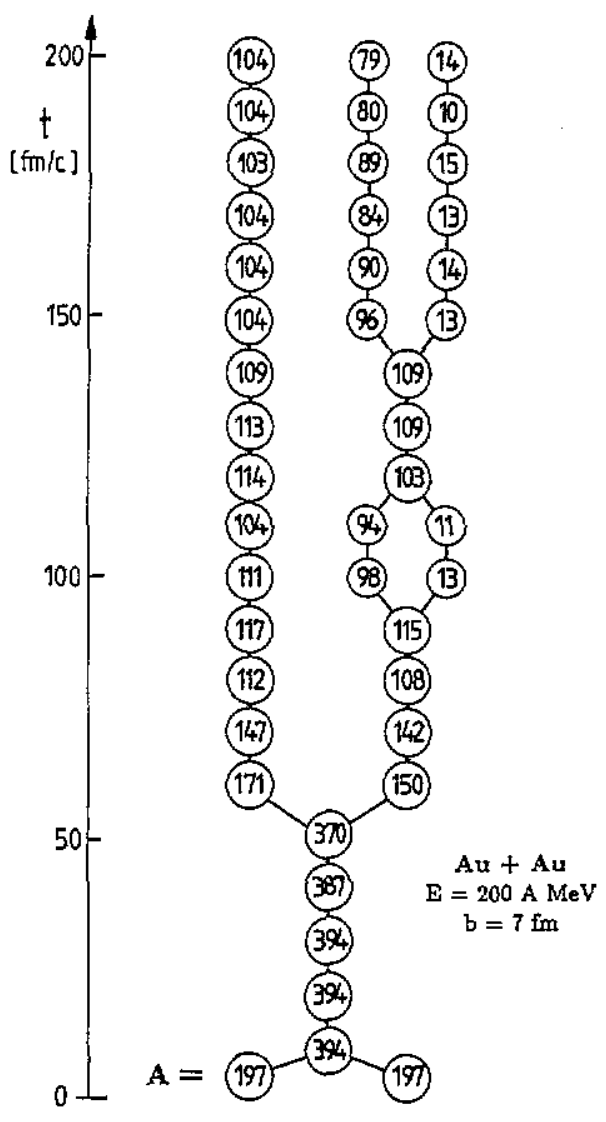

Figure 37. Time evolution of the fragmentation process in a single event for the reaction $\mathrm{Au}$ (200A MeV, $b=7 \mathrm{fm})+A \mathrm{u}$. All massive fragments $(A \geqslant 10)$ from $t=0 \mathrm{fm} c^{-1}$ (bottom) to $t=200 \mathrm{fm} c^{-1}$ (top) are shown. Two nucleons are considered to be members of a fragment if their spatial distance is less than $\alpha_{1}=3 \mathrm{fm}$ (Peilert et al 1989).

at $150 \mathrm{fm} c^{-1}$ or even earlier. Let us first concentrate on the heavy clusters with $A>70$. Here one recognizes one cluster up to $50 \mathrm{fm} c^{-1}$ which is not stable and decays rapidly. The decay chain can be seen by the subsequent population and depopulation of the different mass bins for the smaller clusters. Along the decay chain the cluster also evaporates single nucleons and therefore these numbers increase but have almost saturated at $t=200 \mathrm{fm} c^{-1}$. The clusters in between $2 \leqslant A \leqslant 30$ have a completely different history. They are formed at a very early stage of the reaction and are not fed from the decaying remnants nor do they decay. After $100 \mathrm{fm} c^{-1}$ practically all of them have been formed. They emerge from the surface region of the combined system (Aichelin et al 1988) and measure the violence of the reaction.

Keep in mind that the transient appearance of large 'clusters' reflects the simple configuration space method used to define the clusters. The actual phase-space distributions indicate that the cluster correlations are established much earlier. This can be seen in the transverse momentum transfer discussed above. The complex fragments are most sensitive to the detailed dynamics during the early compression stage. The transverse momentum transfer, which is build up during the expansion from the high density stage, can be seen most clearly for the IMFs. This indicates that these fragments have been formed early as prefragments in the shock zone and therefore show this strongly enhanced sensitivity on the EOS.

The time evolution of the local density in the central region of the reaction is also shown in figure 38. Observe the compression shock at $t \approx 20 \mathrm{fm} c^{-1}$. At this time the highest 


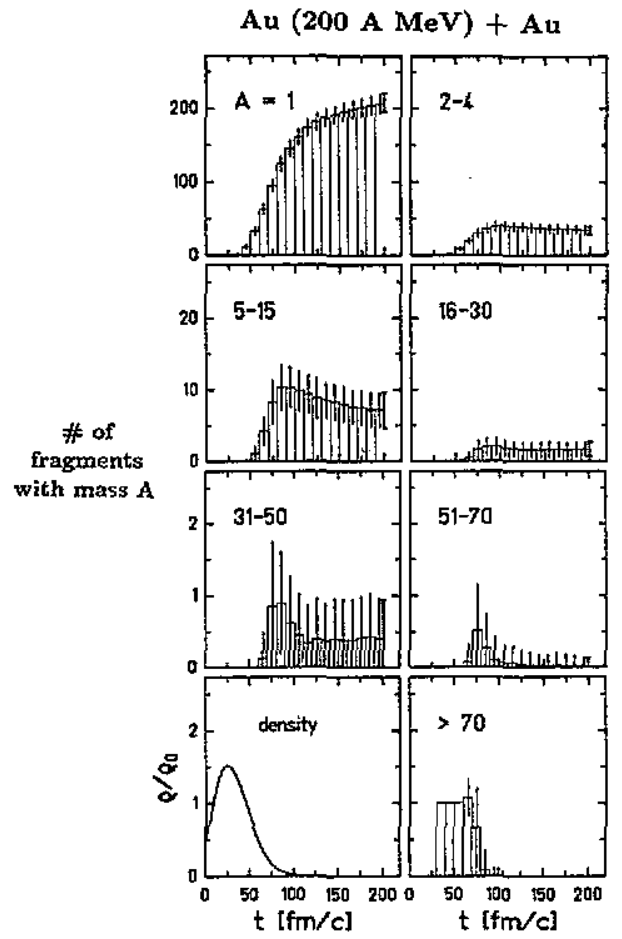

Figure 38. Time dependence of the fragment yields for the reaction $\mathrm{Au}(200 \mathrm{AMeV}, b=3 \mathrm{fm})+\mathrm{Au}$, based on 200 collisions. The average number of fragments for different mass classes as indicated are shown as a function of reaction time. The width of the fragment distributions are indicated by the error bars. Also shown is the time evolution of the local density in the central region of the reaction (Peilert et al 1989).
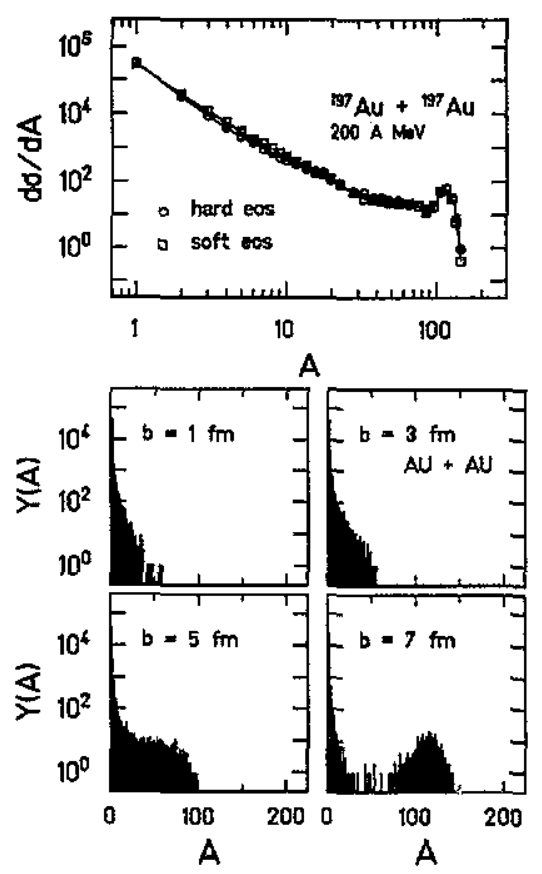

Figure 39. Inclusive, i.e. impact parameter averaged mass yield for the reaction $\mathrm{Au}(200 \mathrm{~A} \mathrm{MeV})+\mathrm{Au}$ obtained with the soft (squares) and the hard (circles) Eos, respectively (upper part). The lower part shows the impact parameter dependence of the average mass yield per event $Y(A)$ for the same reaction (Peilert et al 1989).

temperatures are also found (cf figure 29). Subsequently the system rapidly expands out of the central region of the shocked matter. Note that the highest compression occurs at a time when the fragments are not yet separated in the configuration space. After the central density has decreased they are visible as individual entities only.

Figure 39 shows in the upper part the inclusive, i.e. impact parameter averaged mass yields, for the hard and the soft EOS for $\mathrm{Au}(200 \mathrm{~A} \mathrm{MeV})+\mathrm{Au}$. Both curves exhibit a clear power-law behaviour $Y(A) \sim A^{-\tau}$. The fragment yields, however, are not sensitive to the underlying EOS. For the constant $\tau$ we find $\tau \approx 2.3$. The same behaviour is found in the asymmetric system $\mathrm{Ne}+\mathrm{Au}$ at $1 \mathrm{~A} \mathrm{GeV}$ bombarding energy (Warwick et al 1983 , Aichelin et al 1988). Such a power-law dependence with an exponent $2 \leqslant \tau \leqslant 3$ has been interpreted as evidence for a liquid-vapour phase transition (Minich et al 1982, Panagioutou et al 1984, Csernai and Kapusta 1986). In the present fully dynamic model we can investigate to what extent this conclusion is conclusive. The lower part of figure 39 displays the dependence of the final fragment yields on the impact parameter. In all cases we observe a steep drop of the yields of fragments with $A \leqslant 10$. Large differences become evident for the heavier fragments. For central collisions $(b=1 \mathrm{fm})$ the projectile and target are completely disintegrated and hence there are no $A>40$ fragments. For $b=5$ frn the distribution 
exhibits a flat plateau between $A=40-70$. At $b=7 \mathrm{fm}$ a U-shaped curve with a peak at $A \approx 120$ (projectile and target residue) and almost no fragments in the $A=20-80$ region results. Hence we conclude that impact parameter averaging (rather than a liquid-vapour phase transition) leads to an accidental power-law dependence of the inclusive mass yield in high-energy collisions.

In figure 40 we compare the filtered multiplicity distributions of central and peripheral collisions $(b=3,10 \mathrm{fm}$ ) directly with the corresponding data of Doss et al (1987). The data are shown for those events with maximal (MUL5) and minimal (MUL1) participant proton multiplicity $N_{\mathrm{p}}$. The data and the theory are shown for fragments with $A>5$. Our calculation shows that the mean IMF multiplicities are reproduced quite well within the QMD mode1.

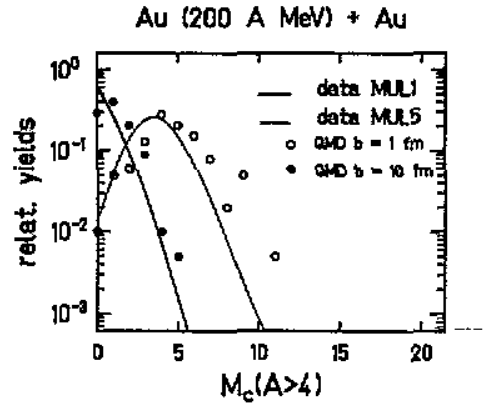

Figure 40. Calculated against measured fragment multiplicity distributions for peripheral (MUL1, $b=$ $10 \mathrm{fm}$ ) and central (MUL5, $b=1 \mathrm{fm}$ ) collisions of the system Au (200A MeV) + Au. The QMD results (circles) (Peilert et al 1989) have been obtained with the Plastic Ball filter while the curves represent the experimental data, taken from (Doss et al 1987, Kampert 1989).

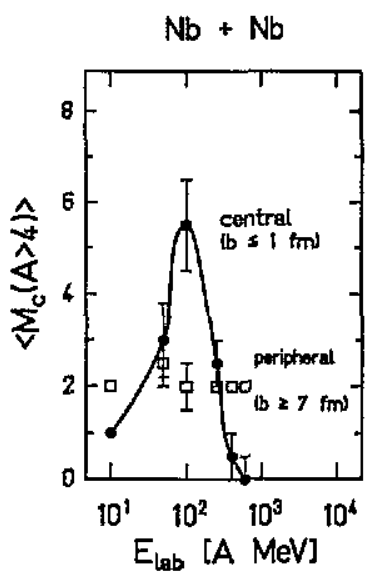

Figure 41. Excitation function of the average multiplicity of IMFs $M_{c}(A>4)$ for the system $\mathrm{Nb} \div$ $\mathrm{Nb}$ obtained without filters for central and peripheral collisions as indicated. The curves are to guide the eye only (Peilert et al 1989).

The IMF multiplicities depend strongly on the bombarding energy. The average multiplicities of IMFS ( $A>4$, without filter) is shown in figure 41 for the $\mathrm{Nb}+\mathrm{Nb}$ system as a function of bombarding energy, for the most central and the peripheral $(b=7 \mathrm{fm})$ collisions. Observe that for central collisions the average multiplicity $\left\langle M_{\mathrm{c}}(A>4)\right\rangle$ is one at low energies (fusion) and has a maximum of about 5 to $6 \mathrm{MFs}$ at intermediate energies $(E \approx 100 \mathrm{AMeV}$ ). This clearly demonstrates that the system completely breaks up into the lightest $(A<4)$ fragments at higher energies.

All the results presented up to now have been obtained with the original QMD model, without the Pauli potential. Since it is basically important for the following considerations to know the excitation energy of the fragments as well, we use from now on the modified QMD approach which also includes the Pauli potential of (Peilert et al 1991) (see section 3 , equation (44) and the discussion there). Within this modified QMD model the nuclei have well defined ground states, including the Fermi motion of the nucleons. Without the Pauli potential the ground state of the Hamiltonian is always a configuration where all the 
momenta are identically zero. As a result we are also now able to calculate the excitation energies of the fragments with respect to their ground states event-by-event.

For the remainder of this review we therefore use the modified model. Before we study the multifragmentation process in more detail, we first investigate the pre-equilibrium processes in such reactions.

Pre-equilibrium neutron emission has been measured recently for the inclusive reactions $\mathrm{p}+\mathrm{Al}, \mathrm{Zr}, \mathrm{Pb}$ at $E_{\mathrm{p}}=80,120$ and $160 \mathrm{MeV}$ (Scobel et al 1990) as well as for 256 and $800 \mathrm{MeV}$ (Stamer 1992). It has been reported (Blann and Vonach 1983, Blann et al 1984, Gruppelaar et al 1986, Trabandt et al 1989) that in this energy regime semiclassical preequilibrium models, which are based on an intranuclear nucleon-nucleon collision process, fail to reproduce the angular distributions. In order to describe the data more appropriately a multistep model based on the Feshbach-Kerman-Koonin (FKK) (Feshbach et al 1980) formalism with at least two or more incoherent direct nucleon-nucleon interactions has been used (Scobel et al 1990). Since the QMD model follows the trajectories of all nucleons microscopically and treats both the hard nucleon-nucleon scattering and the soft nucleonnucleon interaction, it should be equally successful in describing these reactions.

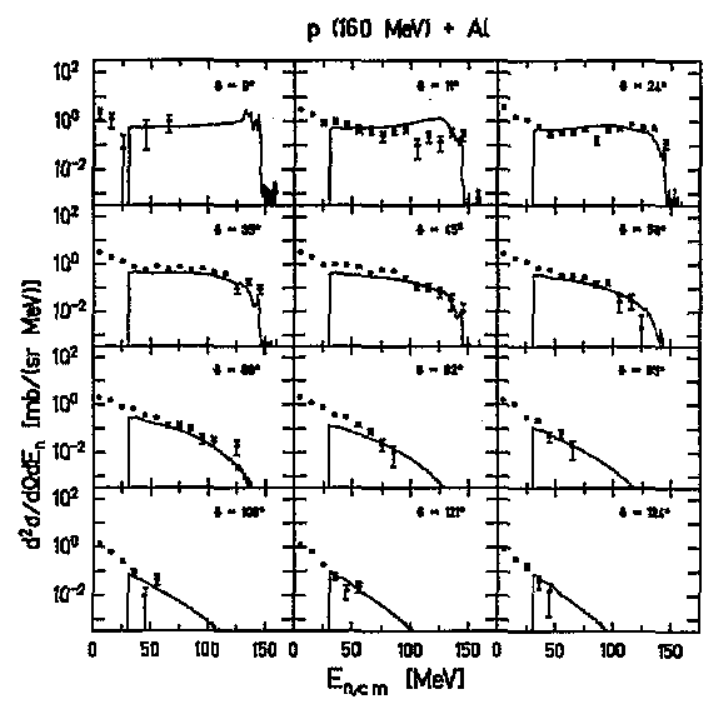

Figure 42. Neutron energy spectra in the $\mathrm{CM}$ frame for the reaction $\mathrm{p}(160 \mathrm{MeV})+\mathrm{Al}$ at different laboratory angles as indicated (Peilert et al 1992). The symbols show the QMD calculation while the full curve represents the data of Scobel et al (1990).

The experimental and calculated $\mathrm{CM}$ energy spectra of neutrons emitted at different laboratory angles are shown in figure 42 for the $\mathrm{Al}$ target at $160 \mathrm{MeV}$ incident proton energy (a detailed discussion of this topic can be found in Peilert et al (1992)). Both the data and the calculations show a characteristic transition from a weak neutron energy dependence at forward angles to an almost exponential shape at backward angles. This clearly indicates that the system is not equilibrated. So we conclude that the failure of the previous pre-equilibrium models to describe the angular dependence of the data is due to the neglect of second- and higher-order collisions. The treatment of the collisions within the quantum mechanical model of Feshbach et al gives a similar agreement with the data, 
which implies that the quantal treatment of the statistical multistep direct emissions is well reproduced with the stochastic collision term in the QMD model. The detailed form of the nucleon-nucleon potential, which is the essential input for, for example, the FKK theory, seems to be unimportant at these high energies. A detailed investigation of the high-energy reactions at 256 and $800 \mathrm{MeV}$ bombarding energies has not so far been achieved. It will, however, be of basic interest to compare all available microscopic models with this type of experiment in order to check the basic input of the models, namely the treatment of the nucleon-nucleon collisions. One expects that the spectra of the emitted neutrons will be very sensitive to the underlying input, especially to the treatment of the produced mesons.

Let us now present a comparison between data and calculations of IMF production in the bombarding energy region between 50 and $100 \mathrm{~A} \mathrm{MeV}$, where the maximum IMF yields are expected to occur (Hahn and Stöcker 1988, Bondorf et al 1985a, Peilert et al 1989).

The data we want to discuss in the next two figures were obtained using 50 and $100 \mathrm{~A} \mathrm{MeV}$ iron beams from the LBL BEVALAC and the PAGODA detector array (Fowler et al 1989). A detailed discussion of the experimental set-up, as well as the inclusive data, are contained in a recent publication (Sangster et al 1992). An important aspect of the experiment is that the correlations between several fragments, emitted from the same reaction, can be measured simultaneously. This allows quite different processes to be uniquely identified.

Inclusive distributions from this experiment do not permit an unambiguous separation of fragments stemming from true multifragmentation (central collisions) and binary fission (peripheral collisions). However, anticorrelations of IMFs with projectile fragments and correlations with the multiplicity of associated protons show (Sangster et al 1992) that fragments with $Z<20$ come almost exclusively from central high multiplicity events. Coincidences between two fragments with $Z>20$ clearly isolate fission, whereas mixed coincidence events $\left(Z_{1}<20\right.$ and $\left.Z_{2}>20\right)$ are dominated by multifragment emission.

For the theoretical description of these reactions we use the modified QMD approach to describe the initial non-equilibrium stage of the reaction. Since the Pauli potential provides a well defined ground state for each fragment we calculate the excitation energy of the prefragments and use this information, together with their mass and charge, as input for the statistical multifragmentation model (SMM) of Botvina et al $(1987,1990)$, that describes the secondary decay of the excited fragments. In the calculation presented below, we stopped the QMD calculations at $300 \mathrm{fm}^{-1}$ reaction time. At this time the fast pre-equilibrium processes are finished and the multiplicities and excitation energies of the prefragments do not change rapidly with time (Peilert 1992). At this time the central densities of the excited fragments in the QMD model are about half the nuclear matter density and also do not change with time (Peilert 1992). This values are consistent with the freeze-out density used in the SMM model. Up until now a precise matching of the density of the prefragments obtained within the QMD with the freeze-out density of the SMM model has not been done, but we use the standard values for all the parameters of the SMM model.

Figure 43 shows the inclusive charge distributions at laboratory angles of $36^{\circ}, 72^{\circ}$, $108^{\circ}$ and $144^{\circ}$ relative to the beam axis for 50 and $100 \mathrm{~A} \mathrm{MeV} \mathrm{Fe}+\mathrm{Au}$ reactions. The cross sections were obtained by integration of the velocity spectra above a threshold of $1.0 \mathrm{~cm} \mathrm{~ns}^{-1}$; no extrapolation below that threshold was performed. The most striking feature is that the basic shape of the distributions does not change as the beam energy is doubled. However, the slopes of the distributions decrease as the beam energy is increased.

A rather dramatic increase in the element yields at large laboratory angles is illustrated in figures 44 which show the angular distributions of several elements for both the 50 and $100 \mathrm{~A} \mathrm{MeV} \mathrm{Fe}+\mathrm{Au}$ reactions. Here we observe an exponential decrease in the cross 
Fe $(50$ A MoV $)+A u$ Fo $(100$ A MoV $)+A u$

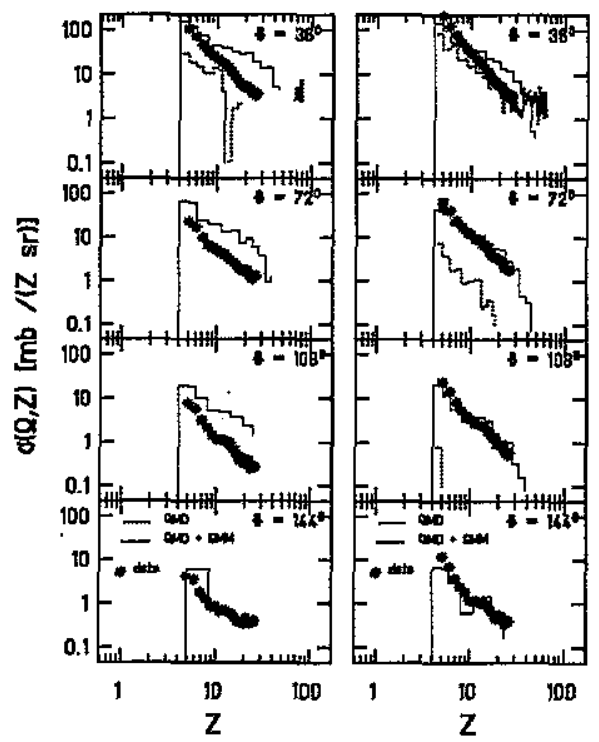

Figure 43. Charge yields for the reactions $\mathrm{Fe}$ $(50,100 \mathrm{~A} \mathrm{MeV})+\mathrm{Au}$ for central collisions at different laboratory angles as indicated. The crosses show the data of Sangster et al (1992) while the histograms represent the QMD calculations obtained from impact parameters 0 to $6 \mathrm{fm}$. The dotted histograms show the results from the QMD model alone while the full histograms refer to the results after the secondary decay of the excited prefragments.

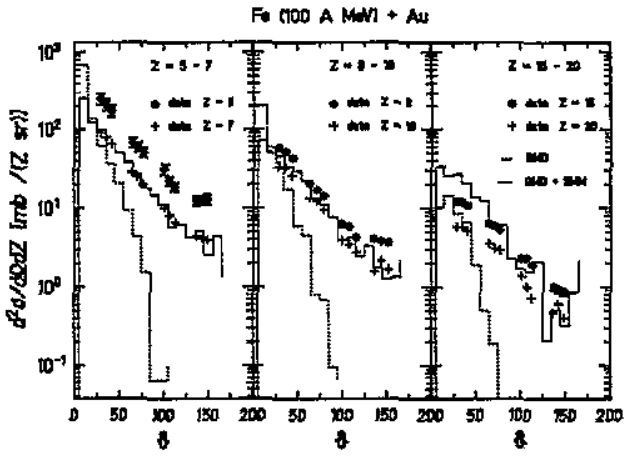

F. $150 \mathrm{~A} \mathrm{Man} \mathrm{+} \mathrm{AN}$

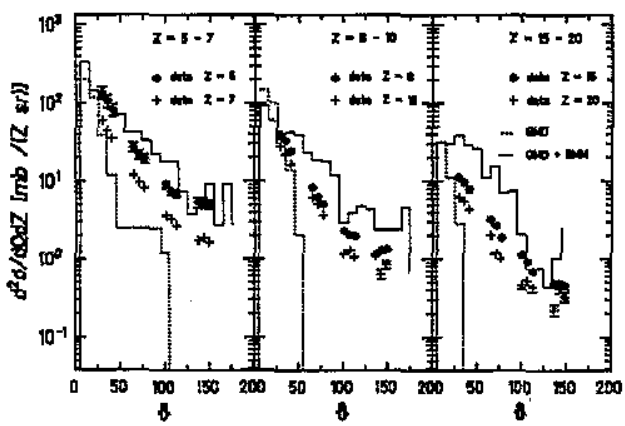

Figure 44. Angular distributions for the reactions $\mathrm{Fe}$ $(50,100 \mathrm{~A} \mathrm{MeV})+\mathrm{Au}$. The data shown are for several charge yields as indicated while the results of the QMD calculations (dotted histograms for the QMD results, full histograms for the results after the secondary decay of the excited prefragments) are averaged over 3 , respectively 6 charge bins.

section with laboratory angle for all elements at both energies. The angular distributions of all elements are steeper at $50 \mathrm{AMeV}$ than at $100 \mathrm{~A} \mathrm{MeV}$. This behaviour is contrary to what one would expect from kinematic focusing.

Together with the data we show in the two figures the results for central collisions $(b=0$ to $6 \mathrm{fm}$ ) obtained with the QMD model alone (dotted histograms) and after the secondary decay of the hot primordial fragments (full histograms). In the output of the calculations the same velocity cut-off of $1.0 \mathrm{~cm} \mathrm{~ns}^{-1}$ has been used. Several heavy prefragments $(Z>60)$ can be seen in the primordial distribution at the most forward angle for the $50 \mathrm{AMeV}$ calculation (figure 44). These fragments are highly excited, decay within the SMM stage and are, therefore, not seen in the final distribution. These heavy primordial fragments are not seen at the higher energy because they are boosted to very forward angles.

Although this model now produces a slight excess of fragments, especially at forward angles and the lower bombarding energy, the qualitative agreement with the data is remarkable. Virtually all of the IMFs observed at backward angles stem from the secondary decay of highly excited heavy-target remnants. The success of this model is perhaps most striking in figure 44 , which shows that the experimentally observed yields at backward angles can only be described by the emission of IMFs due to evaporation from a highly excited target residue. The fast, direct emission of hot fragments from the improved QMD 
model alone produces considerable yields only at the most forward angles. The two-step picture also accounts for the flattening of the angular distributions of the IMFS with beam energy: higher excitation energies boost the IMFs beyond the velocity threshold.

Beside this semi-exclusive experiment and the Plastic Ball experiment, which has been described above, several other multifragmentation experiments have been performed recently. The first of this experiments has been performed at the K1200 Cyclotron at the National Superconducting Cyclotron Laboratory at MSU. The systems investigated there have been $\mathrm{Xe}(50 \mathrm{AMeV})+\mathrm{Au}$ (Bowman et al 1991) and $\mathrm{Ar}(35,50,80,110 \mathrm{AMeV})+$ $\mathrm{Au}$ (Kim et al 1991, de Souza et al 1991). The MSU Miniball Detector, which was used in these experiments, covers approximately $87 \%$ of $4 \pi\left(\vartheta=16-160^{\circ}\right)$ with 171 modules. In addition, a forward hodoscope was used to cover the angles from $\vartheta=1-16^{\circ}$ with 16 elements. This set-up was used to detect fragments with $1 \leqslant Z \leqslant 20$ in the Miniball and $1 \leqslant Z \leqslant 54$ in the forward hodoscope with thresholds of $2,3,4 \mathrm{AMeV}$ for fragments with $Z=3,10,18$ in the Miniball and $6,13,21$ and $27 \mathrm{AMeV}$ for $Z=2,8,20$ and 54 in the forward hodoscope.

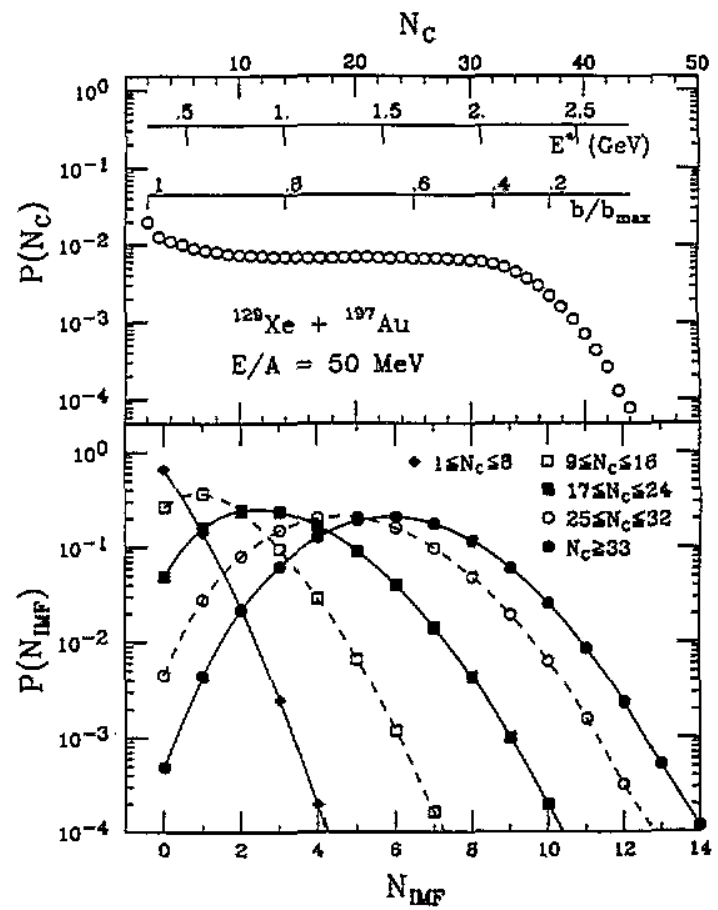

Figure 45. Experimental multiplicity distribution for fragments with $Z=3-20$ in the reaction $\mathrm{Xe}(50 \mathrm{AMeV})+\mathrm{Au}$ (from (Bowman et al 1991)).

Figure 45 shows the (uncorrected) experimental probability distributions in the reaction $\mathrm{Xe}(50 \mathrm{~A} \mathrm{MeV})+\mathrm{Au}$ (taken from (Bowman et al 1991)). The upper part shows the probability distribution for the total charged-particle multiplicity $\left(N_{c}\right)$. A rough scale for the impact parameter and the energy deposition is also shown. The lower part shows the multiplicity distributions of IMFs $(Z=3-20)$ for different $N_{c}$ bins. It can be seen (as 
has been found from Doss et al (1987) for the system $\mathrm{Au}(200 \mathrm{AMeV})+\mathrm{Au})$ that the multiplicity of IMFs increases with decreasing impact parameter.

Before the QMD calculations can be compared with these data, the theoretical events have to undergo an experimental filter. This was not necessary in the semi-exclusive $\mathrm{Fe}$ + Au reactions described earlier, since there the data have been corrected with respect to the detector efficiency, so the only filter was a low velocity cut-off. In this, more general case, there are, in principle, two possibilities why a fragment has not been detected. First, the energy of the fragment could have been too small and second, two or more fragments could have been detected in the same detector module, which would have lead to a misinterpretation of the signal. In the following comparison of the data with QMD calculations we have considered these effects.

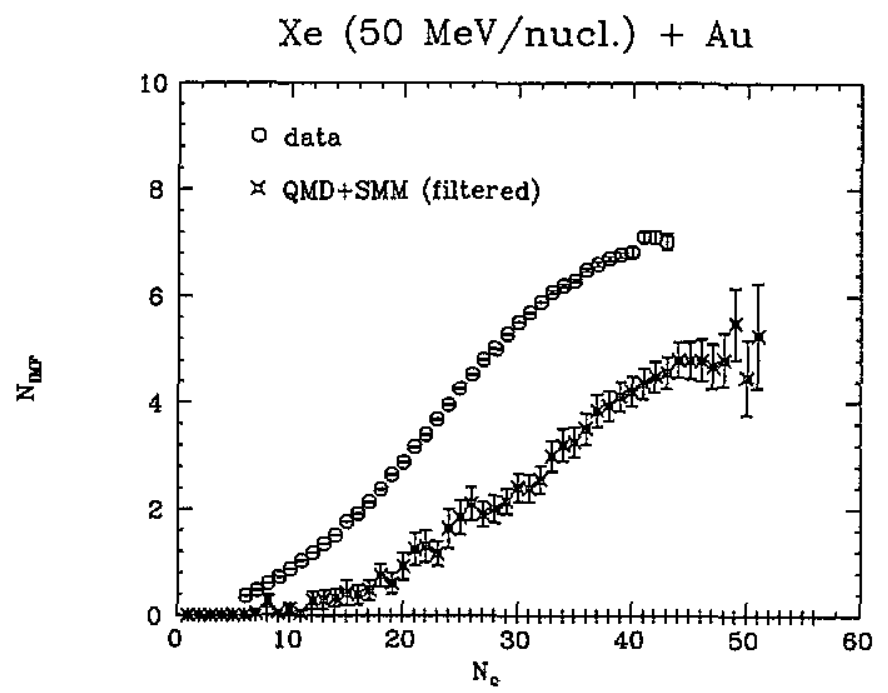

Figure 46. IMF multiplicity as a function of the total charged particle multiplicity $N_{\mathrm{L}}$ in the reaction Xe (50A MeV) + Au. The experiment (Bowman et at 1991) is compared with the QMD + SMM calculations.

In figure 46 we show the multiplicity distributions of MMs as a function of the total charged particle multiplicity $N_{\mathrm{c}}$. Observe the almost linear increase in the fragment multiplicities with increasing charged particle multiplicity (decreasing impact parameter). The highest IMF multiplicities are observed for the most central collisions! The QMD+SMM calculations show the same functional behaviour as the data, but the total IMF multiplicities are about $20-30 \%$ too low.

In the preceding sections we have seen that the production mechanism for IMFs is still one of the unknown properties in such reactions. In high-energy, symmetric collisions (e.g., $\mathrm{Au}+\mathrm{Au}$ at $200 \mathrm{AMeV}$ incident energy) the fragments seem to stem from a dynamical rupture of the system while at lower energies and in asymmetric systems (e.g. Fe/Xe $+\mathrm{Au}$ at $50 / 100 \mathrm{~A} \mathrm{MeV}$ incident energy) it looks more like a 'nuclear boiling', where a highly excited prefragment is formed which then disintegrates into nucleons and fragments.

The transition between these two mechanisms has recently been investigated experimentally by the MSU Miniball group (Tsang et al 1993a, b). For this purpose they 
studied both the systems $\mathrm{Kr}+\mathrm{Au}$ at bombarding energies from 35 up to $400 \mathrm{AMeV}$ and $\mathrm{Au}$ + Au at 100,250 and $400 \mathrm{~A} \mathrm{MeV}$ bombarding energy (the experimental data for the $\mathrm{Kr}+\mathrm{Au}$ experiment are not yet final and therefore cannot be shown here). Previous QMD calculations (see figure 41) have shown that a maximum IMF yield is expected in the bombarding energy region around $100 \mathrm{AMeV}$. These calculations have, however been done with the original QMD, neglecting the secondary decay of the excited prefragments. In order to investigate this transition from low-energy 'thermal multifragmentation' to 'mechanical multifragmentation' at higher energies we used the QMD+SMM approach and calculated the reactions $\mathrm{Kr}+\mathrm{Au}$ at $35 / 55 / 70 / 100 / 200 / 400 \mathrm{~A} \mathrm{MeV}$ bombarding energy for central collisions. In the upper panel of figure 47 we show the average IMF multiplicities $(Z=3-20)$ for those reactions before and after the SMM stage for very central collisions $(b<3.5 \mathrm{fm})$. One observes that the QMD alone reproduces the qualitative structure of this curve that has been observed for the system $\mathrm{Nb}+\mathrm{Nb}$ before (see figure 41 ). After the secondary decay of those fragments this result, however, changes significantly.
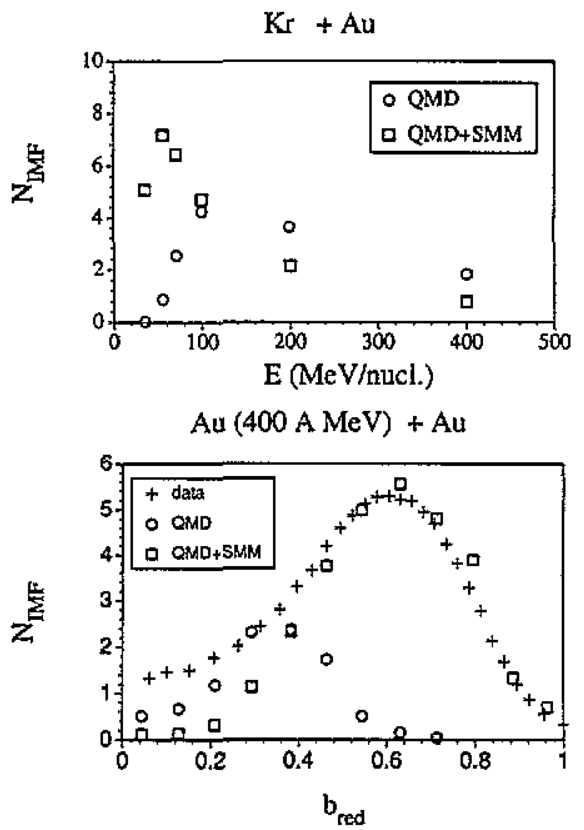

\begin{abstract}
Figure 47. IMF multiplicities (unfiltered) for the reaction $\mathrm{Kr}+\mathrm{Au}$ at bombarding energies from 35 to $400 \mathrm{AMeV}$ (upper panel) for the most central collisions $(b<3.5 \mathrm{fm})$. The calculations have been done using the $\mathrm{QMD}$ and the $\mathrm{QMD}+5 \mathrm{MM}$ approach as indicated. The lower pancl shows the dependence of the IMF multiplicity ( $Z=3-30$ ) on the reduced impact parameter $b_{\text {red }}$ both for the experimental data (crosses) of Tsang et al (1993a) and the QMD (circles) and QMD+SMM (boxes) calculations.
\end{abstract}

The SMM increases the yield of IMFs at low energies due to the explosion of the hot source that is formed at those energies. At higher energies, however, no target residue is formed, but the direct reaction by itself leads to many fragments that are still excited. The secondary decay of these excited fragments reduces the total yield of IMFs. The preliminary data (Tsang et al 1993b) (not shown) show that the peak in the MF multiplicity indeed occurs at about $100 \mathrm{~A} \mathrm{MeV} \mathrm{bombarding} \mathrm{energy.}$

The failure of the QMD+SMM approach to reproduce the decrease in IMF yield at energies below $100 \mathrm{AMeV}$ is possibly due to the fact that the excitation energy of this compound system is too large, because the nucleons are trapped inside the nucleus and cannot escape because of the large Yukawa potential, so the main cooling mechanism (pre-equilibrium nucleon emission) is suppressed in the QMD calculations. 
A more detailed comparison with experimental data can be found in the lower panel of figure 47 for the reaction $A u+A u$. Here the dependence of the IMF multiplicity $(Z=3-$ 30) the reduced impact parameter $b_{\text {red }}$ both for the experimental data (crosses) and the filtered QMD (circles) and QMD+SMM (boxes) calculations is shown. To allow a quantitative comparison between experiment and model calculation it was assumed that the charged particle multiplicity $N_{\mathrm{c}}$ would depend monotonically upon the impact parameter, and a reduced impact parameter $b_{\text {red }}$

$$
b_{\text {red }}=\frac{b}{b_{\max }}=\left[\int_{N_{\mathrm{c}}(b)}^{\infty} \mathrm{d} N_{\mathrm{c}} P\left(N_{\mathrm{c}}\right)\right]^{1 / 2}
$$

was assigned to each data point. Here, $P\left(N_{c}\right)$ is the probability distribution for the chargedparticle multiplicty for $N_{\mathrm{c}} \geqslant 4$ and $b_{\max }$ is the mean impact parameter with $N_{\mathrm{c}}=4$. One observes that the QMD alone underpredicts the production of MMS at central collisions and fails totally to reproduce the large IMF multiplicity for peripheral collisions. The combined model QMD+SMM explains the IMF yield for peripheral collisions but also underpredicts the multiplicities for central collisions

The reason for this is quite obvious. The directly produced fragments are still excited up to several $\mathrm{MeV}$ per nucleon, but now the size of these prefragments lies in the range from $Z=2-30$. The SMM, however, was constructed in the same way as all the other statistical multifragmentation models, in order to describe the statistical decay of a large compound nucleus. So it is at least questionable whether the same approach can be applied to the decay of light, excited nuclei. In addition the excitation energy of these prefragments contains, by its definition, not only the thermal energy but also the collective energy which is, for example, hidden in the radial flow energy of the nucleons if the fragment is still expanding. It has to be seen whether more refined evaporation models, which suppress the level densities of the clusters in the continuum, due to restrictions to bound and quasi bound levels (Mustafa et al 1992), yield similar results. These questions have to be solved before the SMM approach is used to describe the final-stage fragmentation at these energies.

All these open questions are currently under investigation, but all the present studies are consistent with the assumption that at these energies the fragments are predominantly produced from the statistical decay of a highly excited source, while at higher energies the direct multifragmentation channel due to the mechanical rupture of the system becomes more and more important. If one wants to investigate the liquid-vapour phase transition in heavy-ion collisions one is, therefore, restricted to either central collisions of (almost) symmetric systems at low energies or to peripheral collisions at high energies.

If one wants to obtain more information about the mechanism of fragment formation in these reactions one has to go one step beyond the single-particle observables and investigate the correlations between nucleons and fragments. Recently it has been shown from Kim et al $(1992 \mathrm{a}, \mathrm{b})$ that the technique of intensity interferometry can be applied to light fragments, in order to extract the time scale of the reaction. For this purpose they used a final-state Coulomb interaction model. This formalism is, however, restricted to the case, where the fragment-fragment correlation function is governed by the final-state Coulomb interaction of the fragment pair under consideration only. Whether this assumption can hold in the case where one has many (up to 10) fragments which may have been produced in a small volume at the same time, is at least questionable. It was also shown, that the Coulomb influence of a heavy, third body could not be neglected when calculating the light fragment correlation function ( $\mathrm{Kim}$ et al 1992a, b). All these limitations do not apply to the QMD calculations. In this case the Hamilton equations of all nucleons are integrated, which 
implies that correlations between all the existing nucleons and fragments are treated in all orders. This is especially important in the case when one deals not only with two IMFs, but with many, that, in addition, are influenced by a huge number of light fragments and protons. In the following we present the fragment-fragment correlation function for the reaction $\mathrm{Fe}(100 \mathrm{AMeV})+\mathrm{Au}$ for central collisions $(b=0-6 \mathrm{fm})$ in comparison to the data of Sangster et al (1993). For the theoretical calculations we again used the two-step model QMD+SMM. After the break-up of the hot target remnant within the SMM model (this takes place after $300 \mathrm{fm} c^{-1}$ reaction time) we neglect the nuclear forces and follow the Coulomb trajectories of the charged particles. This propagation is stopped when the total Coulomb interaction is sufficiently small.

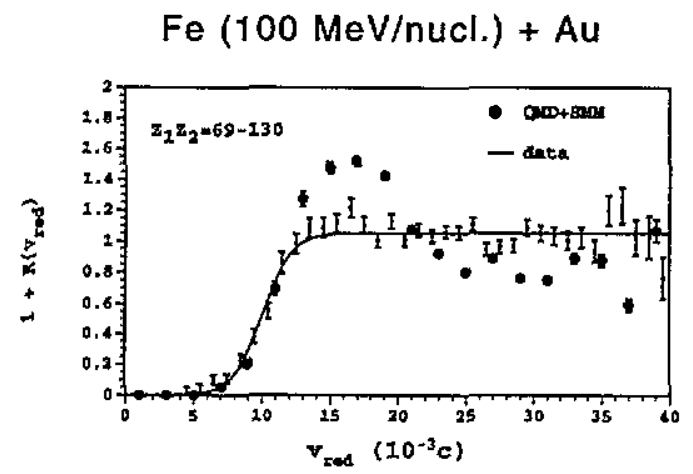

Figure 48. Mixed-fragment reduced-velocity correlation functions IMFs in the the Coulomb product bin $Z_{1} \cdot Z_{2}=65-130$. The experimental results from Sangster et al (1993) are compared with the calculations of the QMD+SMM approach.

The two-fragment correlation function in figure 48 has been constructed by using the technique of event mixing which was first introduced by Kopylov (1974) and later on used by several other authors (Zajc et al 1984, Trockel et al 1987). As the independent variable we use the reduced velocity $v_{\text {ned }}=v_{\text {rel }} / \sqrt{Z_{1}+Z_{2}}$ that has also been introduced also by Kim et al $(1992 \mathrm{a}, \mathrm{b})$. In their work these authors claim that, as nuclear interactions and higher order effects have been neglected, the fragment-fragment correlation function depends only on this scaling parameter. This permits the construction of mixed correlations functions for fragments with different charges. It has, however, been shown (Sangster et al 1993) that this assumption only holds locally, i.e. for small charge bins, which have been under consideration from the MSU group. Figure 48 shows the correlation function $\left(1+R\left(v_{\text {red }}\right)\right)$ for IMFs in the Coulomb product bin $Z_{1} \cdot Z_{2}=65-130$. The effect of the fragment-fragment Coulomb repulsion can clearly be seen, both in the experiment and in the theory. It can be seen from figure 48 , that the QMD+SMM calculations reproduce the size of the Coulomb hole. For larger values of $v_{\text {red }}$ the shape of the calculated results disagrees totally with the experimental curve. While the experiment shows a smooth approach to the asymtotic value of 1 , the calculated curves exhibits a large peak at $v_{\text {red }} \approx 15$. Recent investigations (Peilert et al 1993) show that this peak can be traced back to the more peripheral collisions, where the existence of a large fragment influences the smaller pairs by its Coulomb repulsion. For the present investigation we have used the standard values for the variables in the SMM 
model. These values have been adjusted from the authors of the SMM model to reproduce the results of proton-induced reactions (Botvina et al 1990). In this case a break-up volume of three times the volume of a normal nucleus was needed. It was also found (Peilert $e t$ al 1993) that the size of the Coulomb hole as well as the magnitude of the peak depends on the volume parameter and therewith on the charge asymmetry of the multifragmentation event.

One of the main purposes of future heavy-ion experiments is, therefore, to obtain information about the mechanism of multifragmentation in reactions at this critical energy. The first steps in this direction have already been taken with the first experiments at the new GSI/SIS facility. In these experiments the multifragmentation properties of asymmetric systems at high energies have been investigated by the Aladin group (Hubele et al 1991, Ogilvie et al 1991) and the properties of symmetric systems are under investigation by the FOPI group (Alard et al 1982). The last part of this survey is therefore devoted to the physics investigated by these experiments.

In the very first experiment at the SIS a $600 \mathrm{~A} \mathrm{MeV}$ gold beam was used in combination with the Aladin spectrometer (Hubele 1991, Ogilvie et al 1991). The Aladin spectrometer can basically measure the charge and multiplicity of the heavy-reaction products in the very forward direction $\left(-4.5 \lesssim \vartheta, \varphi \lesssim 4.5^{\circ}\right)$. In addition a hodoscope was used to measure the multiplicity of light particles in the angular regime from $7^{\circ}<\vartheta<40^{\circ}$. Because of the restriction to the very forward direction, study of the reaction was limited to inverse kinematics, i.e. with heavy beams and light targets.

This opens up the possibility of varying the mass of the target in order to change (at fixed beam energy) the violence of the reaction (or, in other words, the total amount of energy which is deposited in the projectile). This was done practically by using a gold beam at $600 \mathrm{AMeV}$ energy and carbon, aluminum and copper as targets.

In this experiments one had to deal-in the same way as in all other heavy-ion experiments-with the problem of how to trigger the impact parameter. It was shown by the Aladin group that for this particular set of experiments a new observable can be used to measure the centrality of the reaction, namely the number of charges which are bound in fragments with $Z>2$ ( $Z_{\text {bound }}$ ). Clearly this observable is complementary to the multiplicity of light particles which is used to focus on centrality in other experiments.

One of the most important results of this set of experiments is shown in the right-hand column of figure 49 . There the correlation of the charge of the largest fragment $\left(Z_{\max }\right)$ with the sum of all bound charges $\left(Z_{\text {bound }}\right)$ is shown for the three different targets. By construction the relation $Z_{\max } \leqslant Z_{\text {bound }}$ must always hold, so all events must result in a point lying below the diagonal. All reactions which yield only one fragment contribute a point close to this diagonal while reactions which produce more than one fragment (the true multifragmentation events) yield points below this diagonal. Note, however, that this representation gives no information about the distribution and multiplicities of the IMFs, since the only information that enters is the total number of charges and the size of the biggest fragment (e.g., a fragmentation in one $Z=50$ and ten $Z=5$ fragments gives the same contribution as the fragmentation in two $Z=50$ fragments).

The right-hand column of figure 49 shows clearly that for very peripheral reactions (large $\left.Z_{\text {bound }}\right)$ there is no multifragmentation, i.e. the gold remains as the largest fragment while with decreasing $Z_{\text {bound }}$ the points shift away from the diagonal, indicating multifragmentation events. At very central collisions with a heavy target a total disintegration of the projectile into light particles is observed, yielding points in the very lower left-hand corner of the plot. Note that these points are absent for the carbon target. In this case there is no 


\section{$\mathrm{Au}(600 \mathrm{MeV} / \mathrm{N})+\mathrm{C}, \mathrm{Al}, \mathrm{Cu}$}

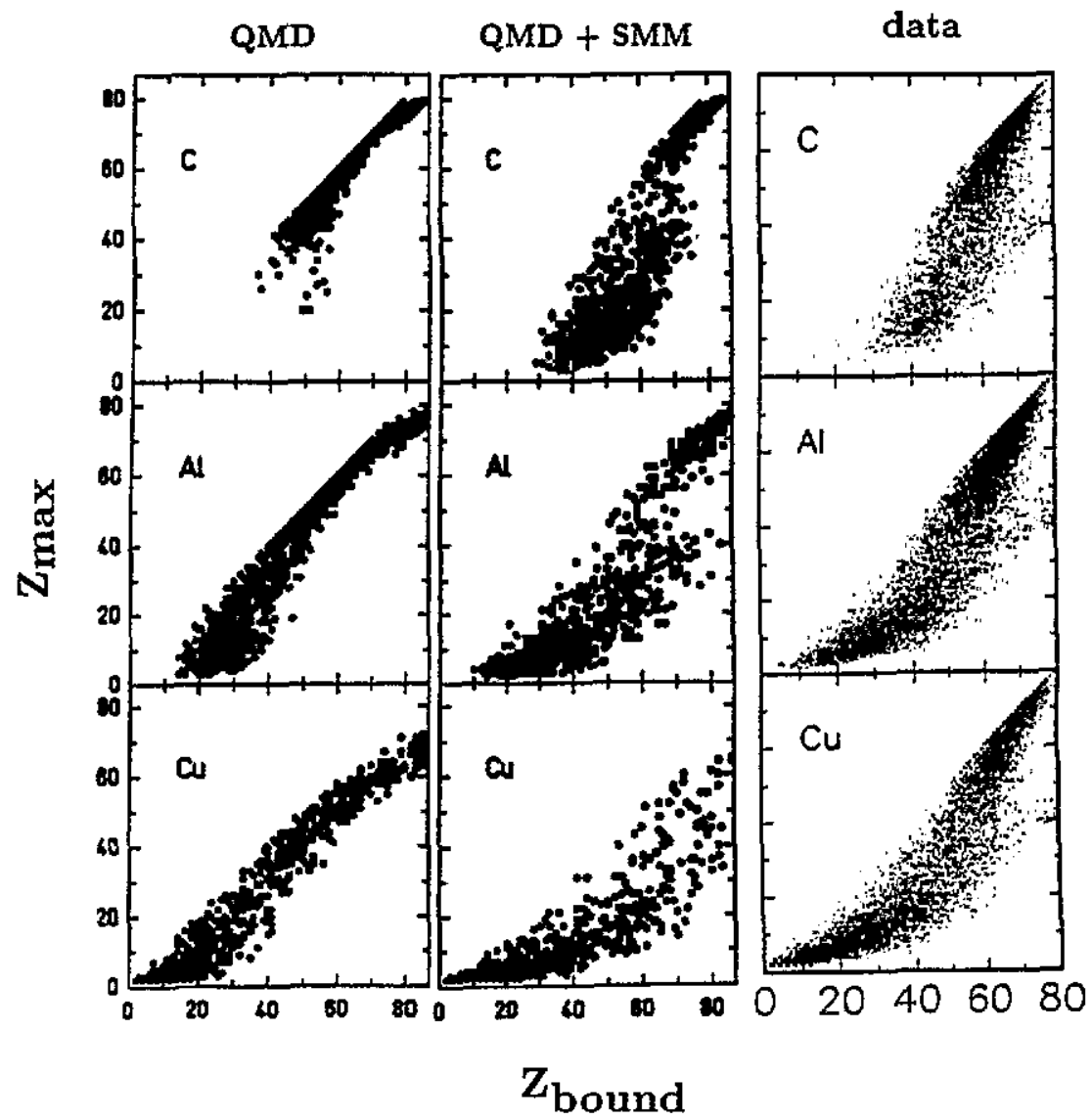

Figure 49. Corrclation between the maximum charge observed within one event $\left(Z_{\max }\right)$ and the total number of charges bound in fragments with $Z \geqslant 2$ ( $Z_{\text {bound }}$ ) for the reactions Au $(600 \mathrm{~A} \mathrm{MeV})+\mathrm{C}, \mathrm{Al}, \mathrm{Cl}$. The calculations performed with the QMD model with (middle column) and without (left-hand column) the secondary decay of the prefragments are compared with the data of Hubele et al (1991).

experimental evidence for a total disintegration of the projectile, but there remains always a heavy projectile rest!

The main information which is missing here, the multiplicity of IMFs, is shown in the right-hand column of figure 50 . Here the multiplicity of the resulting fragments is plotted against the bound charge. The remarking feature here is the fact that the different targets all yield an identical distribution (note, however, that the points with low $Z_{\text {bound }}$ are missing for the light targets).

This leads to the conclusion that $Z_{\text {bound }}$ is not a direct measure of the impact parameter, but more a measure of the violence of the collision or of the deposited energy. A central collision with the carbon target leads to the same prefragment as a more peripheral collision with heavier targets. What is observed in the experiment are, however, the decay products 


$$
\mathrm{Au}(600 \mathrm{MeV} / \mathrm{N})+\mathrm{C}, \mathrm{Al}, \mathrm{Cu}
$$
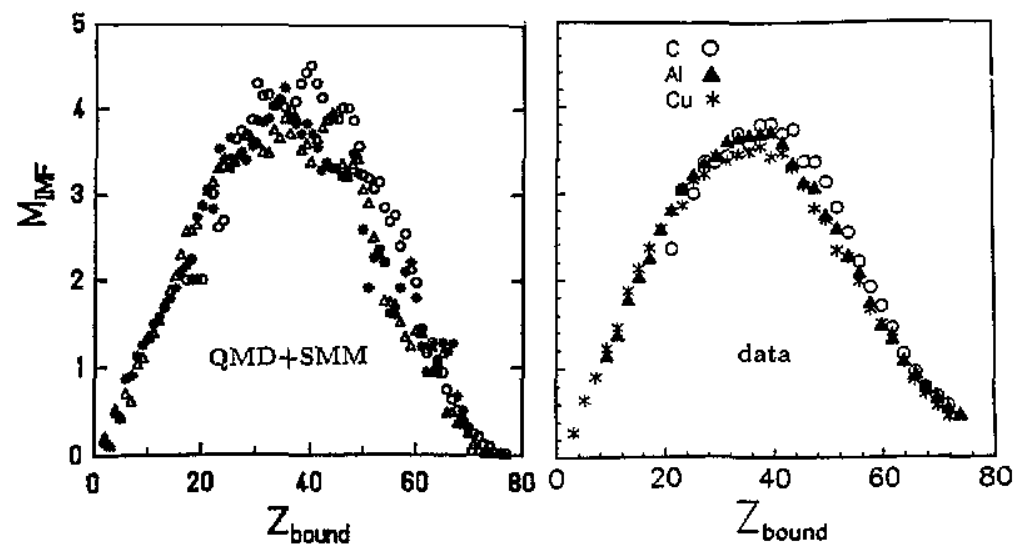

Figure 50. The multiplicity of IMFs $(3 \leqslant Z \leqslant 30)$ in the reaction $\mathrm{Au}(600 \mathrm{AMeV})+\mathrm{C}, \mathrm{Al}, \mathrm{Cu}$ are shown as a function of $Z_{\text {bound }}$ both for the QMD calculation with secondary decay and for the data of Hubele et al (1991).

of this prefragment, which then yield this universal behaviour if plotted against the variable $Z_{\text {bound. }}$

The theoretical results obtained with the QMD model are also shown in figures 49 and 50 (see also Konopka et al 1993). The left-hand column of figure 49 shows the $Z_{\text {max }}-Z_{\text {bound }}$ correlation obtained with the pure QMD model while the middle column shows the same distributions obtained after secondary decay within the SMM. Here one again observes that the QMD model yields in most cases one large excited prefragment (this is especially true for the light target), resulting in an distribution very close to the diagonal. After the secondary decay of these prefragments (middle column of figure 49 ) the distributions agrees much better with the data. This effect can also be seen in the left-hand column of figure 50 , where the multiplicity distributions shown for the QMD+SMM calculations compare nicely with the data, while the pure QMD results (not shown) are completely different.

The basic conclusion from this experiment and the comparison with the theoretical results is the fact that in these asymmetric reactions the IMFS stem from the statistical decay of a highly excited prefragment which is produced in the direct collision. This production mechanism seems to be the dominant channel for the carbon target; for the heavier targets it applies for the peripheral reactions while in central collisions the direct production of IMFS is also important. A similar experiment has recently been performed by the EOS/TPC collaboration at $\mathrm{LBL}$ for the reaction $\mathrm{Au} / \mathrm{Kr} / \mathrm{La}(1 \mathrm{~A} \mathrm{GeV})+\mathrm{C}$ (Hjort et al 1993).

This transition from statistical decay to direct fragmentation is one of the most intriguing features in this field which should be studied in greater detail. For this purpose one needs, however, more information about the reaction than that contained in charge yields and multiplicity distributions. Of particular importance in this context are the momentum space distributions of the IMFs, which could give information about the degree of equilibration, and the (velocity and angular) fragment-fragment correlations, from which one can possibly gain information about the time scale of the fragmentation process.

All this information can be provided by a $4 \pi$ detector. Such a device has been constructed by the FOPI collaboration at the GSI. The basic properties of this detector are:

(i) it covers almost the complete phase space for charged fragments; 
(ii) the granularity is high enough to dissolve high multiplicity events as well;

(iii) the azimuthal symmetry the reaction plane to be reconstructed;

(iv) it allows light particles $\left(\pi^{ \pm}, \mathrm{K}, \mathrm{p}, \mathrm{t},{ }^{3} \mathrm{He}^{4} \mathrm{He}\right)$ to be distinguished;

(v) it can detect fragments up to $Z=20$.

This detector has been constructed in two steps. The first step (called Phase I) was finished in summer 1991 and the second step (Phase II) went into operation at the end of 1992. With Phase I it was possible to cover the forward hemisphere for the reactions $\mathrm{Au}$ $+\mathrm{Au}$ at beam energies from 150 to $800 \mathrm{AMeV}$ with a plastic wall $\left(1 \leqslant \vartheta \leqslant 30^{\circ}\right)$ and the cluster detectors ROSACE $\left(1 \leqslant \vartheta \leqslant 6^{\circ}\right)$ and parabola $\left(6 \leqslant \vartheta \leqslant 30^{\circ}\right)$.

The second large experiment that was devoted to investigating the nuclear EOS at high densities was performed at the BEVALAC from the EOS group, utilizing a detector in the form of a time projection chamber (TPC) (Hjort et al 1993). The EOS/TPC was designed to cope with the high multiplicity events characteristic for reactions with the heaviest and most energetic beams at the BEVALAC. In the EOS experiments at the BEVALAC beams of $\mathrm{Ca}, \mathrm{Ni}, \mathrm{Kr}, \mathrm{La}$ and $\mathrm{Au}$ with energies from $250 \mathrm{AMeV}$ to the maximum possible energy (2.1 $A \mathrm{GeV}$ for $\mathrm{Ca}, 1.2 \mathrm{~A} \mathrm{GeV}$ for $\mathrm{Au}$ ) were used to study both symmetric and asymmetric collisions. In combination with the EOS/TPC the Music II detector was used with a timeof-flight wall in order to identify forward moving particles beyond the dynamic range of the TPC. This combination of detectors identified particles with charges greater than 7 .

The EOS/TPC experiments have been designed to attempt a systematic measurement of the energy and mass dependence of the flow effects and to make a high statistics, exclusive study of nuclear multifragmentation. For this purpose reverse kinematics were used to study projectile fragmentation in the systems $\mathrm{Kr}+\mathrm{C}, \mathrm{La}+\mathrm{C}, \mathrm{Au}+\mathrm{C}$ at $1 \mathrm{~A} \mathrm{GeV}$. Since the results of these experiments have not yet been completely published we cannot present a comparison with the theory right now.

One of the basic aims of these experiments is to shed some light on the nuclear EOS therefore it is of the utmost importance to trigger on very central collisions. This has previously been done with multiplicity triggers only, but it has been shown by the FOPI group (Alard et al 1982) that it is possible to trigger on even more central collisions if one uses not only the multiplicity, but also the phase-space information of the reaction.

The upper part of figure 51 shows the distribution of the total multiplicity of charged particles obtained with the QMD model (without filter). This distribution shows a plateau at intermediate multiplicities and then falls rapidly off going to higher multiplicities. The total multiplicity regime is now divided into five bins, where the highest bin (PM5) contains all the events which yield a multiplicity larger than that at the point where the distribution begins to drop. The remaining interval is then divided into four bins with equal widths called PM1-PM4. For the ideal case, where the multiplicity drops linearly with impact parameter this procedure would give a linear dependence between the impact parameter and the multiplicity bins. This, however, is not the case practically, as central collisions depend only weakly on the multiplicity on the impact parameter. In order to trigger on this very central collisions one uses the following trick (Alard et al 1982).

The main feature of central collisions $(b=0 \mathrm{fm})$ is the complete azimuthal symmetry. For precisely this reason it was not possible to extract the so called 'transverse flow' for very central collisions (see section 4). Now one can turn this relation around and use the azimuthal symmetry of the reaction in order to trigger the centrality. This is done practically by using the transverse-momentum directivity $D$, defined as

$$
D=\left.\frac{\left|\sum_{i} p_{\mathrm{t}}\right|}{\sum_{i}\left|p_{\mathrm{t}}\right|}\right|_{y_{i}>\mathrm{yCM}_{\mathrm{CM}}}
$$




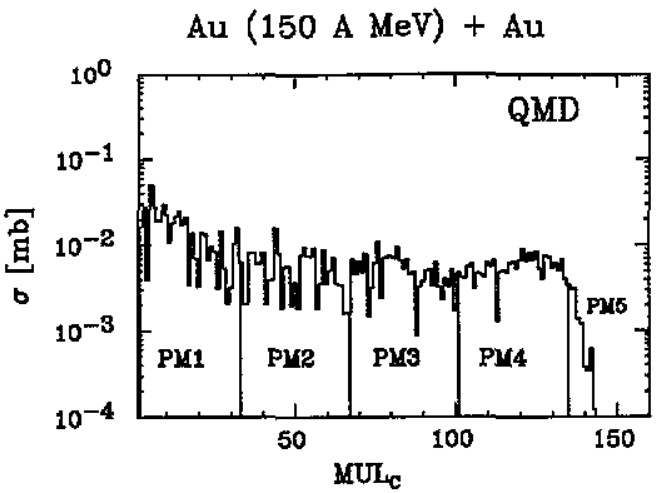

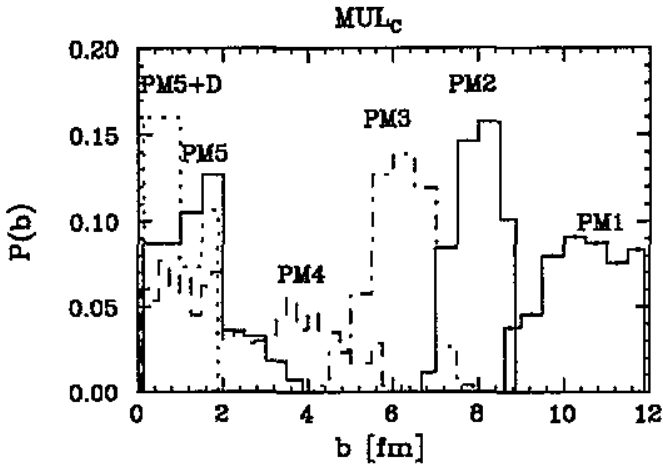

Figure 51. Distributions of the total charged-particle multiplicities in the reaction $\mathrm{Au}(150 \mathrm{AMeV})+\mathrm{Au}$ obtained with the QMD model. The upper figure shows the multiplicity distributions with the cuts PM1PM5 which are used in the experiment to trigger the centrality. The lower figure shows the impact parameter ranges which contribute to the different multiplicity bins. The meaning of the 'directivity trigger' PM5 $+D$ is explained in the text.

where the sum runs over all (charged) particles with $Z \leqslant 15$. For $b=0$ it obviously holds that $D=0$. In addition to the multiplicity bins PM1-PM5 one now uses the trigger PM5 $+D$ which contains all events from the PM5 bin with a $D$ value lower than 0.2 .

The lower part of figure 51 now shows the impact parameter ranges which are, according to the QMD calculations, selected by these triggers. We observe that the peripheral collisions (PM1-PM3) are clearly separated from each other (this, however, is only the case in the theoretical calculation where one does not have to deal with a background) while for central collisions there is a large overlap between the different distributions. PM4 covers the impact parameter range from 0 to $6 \mathrm{fm}$ while PM5 selects the impact parameter region up to $4 \mathrm{fm}$. The additional trigger PM5 $+D$, however, gives an additional cut by selecting only the very central events with $b<2 \mathrm{fm}$.

As a final point we would like to investigate the charge-yield distributions in these reactions. As was pointed out earlier it is hoped that information about the supposed liquid-vapour phase transition could be obtained from the charge distribution in exclusive experiments.

In figure 52 we compare the calculated (filtered) charge-yield distributions for the reaction $\mathrm{Au}(150 \mathrm{~A} \mathrm{MeV})+\mathrm{Au}$ with the experimental data of the FOPI collaboration (Kuhn et al 1993) for the multiplicity bin PM4. Both the results obtained with the QMD (circles) as well as with the QMD+SMM (boxes) approach are shown. It can be seen that the slope of the charge yield distribution is reasonably well reproduced by the QMD calculations, while the QMD+SMM results are steeper than the data (for the same reasons as has been described in the discussion of figure 47). The discrepancy of a factor two that was observed for IMF multiplicities in the $400 \mathrm{MeV}$ Au-Au reaction in figure 47 is also present in this data set but it is almost invisible because of the logarithmic scaling. 


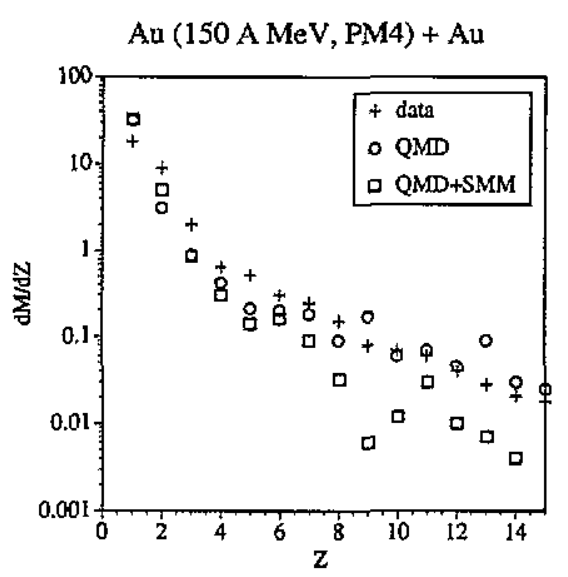

Figure 52. Charge distributions for the reaction $A$ u $(150 \mathrm{~A} \mathrm{MeV})+\mathrm{Au}$ obtained with the QMD and QMD+SMM approach compared with the experimental data of Kuhn et al (1993).

\section{Conclusion}

We have reviewed the present status of heavy-ion physics. The basic concepts of the nuclear EOS have been introduced and the idea of shock compression and heating was summarized. We presented the three main approaches to the theoretical description of heavy-ion collisions that have survived the zoo of models developed in the past decades. These approaches are the nuclear fluid dynamical model and the microscopic models based on the vUU/BUU equation and the molecular dynamical models of the QMD type.

These models have been used to investigate the most important signatures in heavy-ion reactions. The nuclear matter flow, i.e. the in-plane bounce-off and the out-of-plane squeezeout are sensitive to the underlying EOS, but these observables also depend on other unknown properties of nuclear matter such as the in-medium nucleon-nucleon scattering cross section and the momentum dependence of the nuclear forces. To the best of our knowledge there is no experimental evidence for a significant change in the nucleon-nucleon scattering cross sections in the nuclear medium compared with the vacuum cross sections that are used in the QMD and vuU models. The momentum dependence of the nuclear forces has been adapted from the experimental values obtained from the real part of the optical potential in proton-induced reactions. The observables discussed here can therefore be used to extract nuclear matter properties like the ground-state compressibility constant of nuclear matter from the most recent experimental data that are available now. Of particular importance here is the fragment flow, since our calculations clearly show that the fragments show an enhanced sensitivity to the underlying EOS.

In order to study the applicability of macroscopic models we have investigated the stopping power of interpenetrating nuclei, as well as the degree of thermalization found in symmetric collisions. It turned out that neither a one- nor a two-fluid model can hold, since the dynamical evolution of the reaction is dominated by the interaction of the projectile and the target with the participant matter and not with each other. A rapid approach to equilibrium has been found in such a three-fluid scenario.

We discussed the possibility of a liquid-vapour phase transition by a comparison of calculations with the most recent multifragmentation experiments. In these investigations a consistent picture of the reaction process over a broad range of excitation energies in the target residue is beginning to evolve. For very low excitation energies the relative slow fission process is the most important decay channel. At higher bombarding energies a multifragmentation emerges and dominates the residue decay. We have shown that for 
asymmetric reactions the large cross section for MMFs, especially at backward angles, is due to the statistical decay of the excited heavy residue that remains following the initial stages of the reaction. This production mechanism is supported by the experimental data from three different, independent groups, namely the LBL/LLNL Pagoda, the MSU Miniball, and the GSI Aladin group. As the bombarding energy continues to increase, QMD calculations suggest that a rapid compression-decompression mechanism emerges in the initial stage of the reaction and leads to a direct multifragmentation process and a highly excited heavy residue is no longer formed. A comparison with recent $\mathrm{Kr}+$ Au date from the MSU Miniball collaboration suggest that the best region to search for thermally driven multifragmentation is the bombarding energy region around $100 \mathrm{AMeV}$.

A new, potentially interesting key to understanding the multifragmentation process is the study of the correlations in charge and velocity between two or more MMFs emitted from the heavy remnant.

In this review we have restricted ourselves to heavy-ion reactions in the energy regime available at the BEVALAC, the SIS and at MSU. The model calculations show, however, that at those energies the maximum compression that is achieved in central collisions of the heaviest nuclei is about two to three times normal nuclear matter density. Since we are interested in exploring the nuclear EOS to much higher densities it is clear that the bombarding energies at these facilities are no longer sufficient to complete these tasks. The ultimate goal even is to find evidence for densities that are so large, that the constituents of the nucleons, quarks and gluons share a volume larger than the volume of one nucleon and form the so called quark-gluon plasma.

For this purpose experiments have been started at the AGS at the Brookhaven National Laboratory utilizing silicon and gold beams with $14 / 10 \mathrm{~A} \mathrm{GeV}$ bombarding energies and at the SPS at Cern with a sulphur beam at $200 \mathrm{~A} \mathrm{GeV}$ bombarding energy.

Recent investigations with the relativistic extension of the QMD model, the so called RQMD model (Sorge et al 1989, 1991a, b, 1992a, b) have shown that the collective flow is still important even at these high energies. The complete stopping of the collisions partners for central collisions is predicted for all energies available at those accelerators. If information about the nuclear EOS at low and intermediate densities $\left(\rho / \rho_{0}<3\right)$ and at high densities $\left(\rho / \rho_{0} \approx 5-10\right)$ is available it is also of the utmost importance to explore the density/energy regime in between. A detailed excitation function for all the available observables discussed in this review as well as information about pions and produced mesons, dileptons and antiparticles (e.g. $\overline{\text { p}}$ ) from bombarding energies from $1 \mathrm{~A} \mathrm{GeV}$ up to $20-50 \mathrm{~A} \mathrm{GeV}$ could give evidence for the exotic behaviour of the EOS due to density isomers, resonance matter etc that would alter the functional form of the EOS.

\section{Acknowledgments}

We would like to thank Professor Dr J Aichelin, Dr C Hartnack, Dr B Waldhauser, Dr D Rischke, Dr M Berenguer, Dipl. Phys. J Konopka, and Dipl. Phys. S Bass for providing parts of the material as well as for many valuable discussions. Since progress in the field of heavy ion collisions can only be achieved through a deep interplay between theory and experiment, we would like to thank the ALADIN and FOPI groups at GSI, the MSU Miniball group, and the EOS group at LBL, for providing us with their data and for many helpful discussions.

This work was supported in part by the German Federal Ministery for Research and Technology (BMFT), the Gesellschaft für Schwerionenforschung (GSI) and the US 
Department of Energy by LLNL under Contract W-7405-ENG-48. One of us (GP) gratefully acknowledges support from the Wissenschaftsausschuss of the NATO via the DAAD and the Alexander von Humboldt Foundation.

\section{References}

Aichelin J 1991 Phys. Rep. 202233

Aichelin J and Bertsch G F 1985 Phys. Rev. C 311730

Aichelin J, Hartnack C, Bohnet A, Zhaxia L, Peilest G, Stöcker H and Greiner W 1989 Phys. Lett. 22AB 34

Aichelin J, Peilert G, Bohnet A, Rosenhauer A, Stסcker H and Greiner W 1988 Phys. Rev. C 372451

Aichelin J and Stöcker H 1985 Phys. Lett. 163B 59

Aichelin J and Stöcker H 1986 Phys. Lett. 176B 14

Alard J P et al 1987 Nucl. Instrum. Methods A 261379

Alard J P et al 1992 Phys. Rev, Lett. 69889

Aleklett K, Loveland W, De Saint-Simon M, Sihver L, Liljenzin J O and Seaborg G T 1982 Nucl. Phys. A 499 591

Aleklett K, Loveland W, De Saint-Simon M, Sihver L, Liljenzin J O and Seaborg G T 1990 Phys. Lett. 236B 404 Amsden A A, Ginocchio J N, Harlow F H, Nix J R, Danos M, Halbert E C and Smith R K 1977a Phys. Lett. 38 1055

Amsden A A, Harlow F H and Nix J R 1977b Phys. Rev. C 152059

Anastasio M R, Faessler A, Müther H, Holinde K and Machleidt R 1978 Phys. Rev, C 182416

Anastasio M R, Celenza L S, Pong W S and Shakin C M 1983 Phys. Rep. 100327

Bäckmann S O, Clark J W, ter Louw W J, Chakkalakal D A and Ristig M L 1972 Phys. Rev. Lett. B 41247

Barbagallo C, Richert $J$ and Wagner P 1986 Z. Phys. A 32497

Baumgardt H G and Schopper E 1979 J. Phys. G: Nucl. Phys. 5231

Baumgardt H G, Schott J U, Sakamoto Y, Schopper E, Söcker H, Hofmann J, Scheid W and Greiner W 1975 Z Phys. A 273359

Beauvais G E, Boal D H and Wong J C K 1987 Phys. Rey. C 35545

Beavis D, Chu S Y, Fung S Y, Gorn W, Huie A, Keane D, Lu J J, Poe R T, Shen B C and VanDalen G 1983 Phys. Rev, C 272443

Berenguer M, Hartnack C, Peilert G, Stöcker H, Greiner H, Aichelin J and Rosenhauer A 1992 J. Phys. G: Nuct. Phys. 18655

Bertsch G and Amsden A A 1978 Phys. Rev. C 181293

Bertsch G and Das Gupta S 1988 Phys. Rep. 160189

Bertsch G and Siemens P I 1983 Phys. Lett. 12689

Bertsch G, Kruse H and Das Gupta S 1984 Phys. Rev, C 29673

Bertsch G, Lynch W G and Tsang M B 1987 Phys. Lett. 189B 384

Blaizot J P 1980 Phys. Rep, 64171

Blaizot J P 1989 Proc. NATO Adv. Study Inst. Nuclear Equation of State, Peniscola, Spain (NATO ASI Series, Series B) vol 216A, ed W Greiner and H Stocker p 679

Blaizot J P, Gogny D and Grammaticos B 1976 NucL. Phys. A 265315

Blann M 1985 Phys. Rev. Lett. 54 2215; 1985 Phys. Rev. C 32 1231

Blann M and Vonach H K 1983 Phys. Rev. C 281475

Blann M, Scobel W and Plechaty E 1984 Phys. Rev. C 301493

Blann M, Komoto T and Tservaya I 1989 Phys. Rev. C 402498

Blann M, Mustafa M G, Peilert G, Stöcker H and Greiner W 1991 Phys. Rev. C 44431

Boal D H and Glosli J N 1988 Phys. Rev. C 38 1870; 1988 Phys. Rev. 382621

Boal D H, Gosli J N and Wicentowich C 1989 Phys. Rev, C 40601

Bodmer A R 1978 Proc. Symp. Relativistic Heavy lon Research, GSI Darmstadt. March 1978; 1978 Preprint Angonne National Laboratory

Bodmer A R and Panos C N 1977 Phys. Rev. C 151342

Bodmer A R, Panos C and MacKellar A D 1980 Phys. Rev. C 221025

Boguta J and Bodmer A R 1977 Nucl Phys. A 292413

Boguta J and Stocker H 1983 Phys. Lett. 120B 289

Bohnet A, Aichelin J, Peilert G, Stöcker H and Greiner W 1991 Phys, Rev. C 442111

Bonche P, Koonin S and Negele J W 1976 Phys. Rev. C 131226

Bondorf J P, Siemens P J, Garpman S and Halbert E C 1976 Z. Phys. A 279385 
Bondorf J P, Donangelo R, Mishustin I N, Pethick C J, Schulz H and Sneppen K 1985a Nucl. Phys. A 443321 Bondorf J P. Donangelo R, Mishustin I N and Schulz H 1985b Nucl. Phys. A 444460

Botvina A S, Iljinov A S, Mishustin I N, Bondorf J P, Donangelo R and Sneppen K 1987 Nucl. Phys. A 475663 Botvina A S, lljinov A S and Mishustin IN 1990 Nucl. Phys. A 507649

Bowman D R et al 1991 Phys. Rev. Lett. 671527

Brockmann R and Machleidt R 1984 Phys. Lett. 149B 283

Buchwald G, Csernai L P, Maruhn J A, Greiner W and Stöcker H 1981 Phys. Rev. C 24135

Buchwald G, Gräbner G, Theis J, Maruhn J A, Greiner W and Stöcker H 1983a Phys. Rev. C 281119

Buchwaid G, Gräbner G, Theis J, Maruhn J A, Greiner W, Stöcker H, Fraenkel K and Gyulassy M 19836 Phys. Rev. C 282349

Buchwald G, Gräbner G, Theis J, Maruhn J A, Greiner W and Stöcker H 1984 Phys. Rev. Lett. 521594

Callaway D L E, Wilets L and Yariv Y 1979 Nucl. Phys. A 327250

Chapline G F, Johnson M H, Teller E and Weiss M S 1973 Phys. Rev. D 84302

Clare R B and Strottman D 1986 Phys. Rep. 141177

Co G and Speth J 1986 Phys. Rev. Lett. 57547

Csemai L P and Barz H W 1981 Z. Phys. A 296173

Csemai L P and Kapusta J I 1986 Phys. Rep. 131223

Csernai L P, Lukacs B and Zimanyi J 1980 Nuovo Cimento Lett. 27111

Csernai L P, Iovas I, Maruhn J A, Rosenhauer A, Zimanyi J and Greiner W 1982 Phys. Rev. C 26149

Cugnon J 1980 Phys. Rev. C 221885

Cugnon J, Mizutani T and Vandermeulen J 1981 Nucl. Phys. A 352505

Cugnon J, Knoll J, Riedel C and Yariv Y 1982 Phys. Lett. 109B 167

Cugnon J, Lejeune A and Grange P 1987 Phys. Rev. C 35861

Danielewicz P 1984 Phys. Lett. 1468168

Danielewicz P and Gyulassy M 1983 Phys. Lett. 129B 283

Danielewicz $P$ and Odyniec G 1985 Phys. Lett. 157B 146

Day B D 1981 Phys. Rev. Lett. 47 226; 1981 Phys. Rev. C 241203

Day B D and Coester F 1976 Phys. Rev. C 131720

Dickhoff W H, Faessler A and Müther H 1982 Nucl. Phys. A 389492

Dorso $C$ and Randrup $J 1988$ Phys. Lerr. $215 B 611$

Dorso C and Randrup J 1989 Phys. Lett. 232B 29

Doss K G R et al 1986 Phys. Rev. Lett. 57302

Doss K G R et al 1987 Phys. Rev. Lett. 592720

Duer H 1956 Phys. Rev. 103469

Eisenberg $I M$ and Greiner W 1987 Nuclear Theory vol 1, 3rd edn (Amsterdam: Narth Holland)

Fai G and Randrup J 1982 Nucl. Phys. A 381557

Fai $G$ and Randrup J 1983 Nucl Phys, A 404551

Feldmeier H $1990 \mathrm{NuCl}$ Phys. A 515147

Feshbach H, Kerman A and Koonin S E 1980 Ann. Phys. 125429

Finn J E et al 1982 Phys. Rev, Lett. 491321

Fisher M E 1967 Physics 3255

Fowler M M et al 1989 Nucl. Instrum. Methods A 281517

Friedländer E M and Friedmann A A 1967 Nuovo Cimento A $\$ 2912$

Friedman B and Pandharipande V R 1981 Nucl. Phys. A 361502

Friedman W A 1988 Phys, Rev. Lett. 602125

Gale C, Bertsch G F and Das Gupta S 1987 Phys. Rev. C 351666

Garpman I A, Glendenning N K and Karant Y J 1979 Nucl. Phys. A 322382

Gelbke C K and Boal D 1987 Prog. Part. Nucl. Phys. 19c 33

Glendenning N K 1984 Phys. Lett. 144B 158

Goodman A L, Kapusta J I and Mekjian A Z 1984 Phys. Rev. C 30851

Gosset J, Gutbrod H H, Mayer W G, Poskanzer A M, Sandoval A, Stock R and Westfall G 1977 Phys. Rev. C 16 629

Gosset J, Kapusta J I and Westfall G $1978 P h y s, R e \nu, C 18844$

Green A M 1976 Rep. Prog. Phys. 391109

Gregoire C, Remaud B, Sebille F, Vinet L and Raffray Y 1987 Nucl. Phys. A 465317

Gross D H E 1990 Rep. Prog. Phys. 53605

Gross D H E and Zhang X Z 1985 Phys. Lett. 161B 47

Gross D H E, Zhang X Z and Xu S Y 1986 Phys. Rev. Leth 561544

Gruppelaar H, Nagel P and Hodgson P E 1986 Nuovo Cimento 91 
Gudima K K and Toneev V D 1978 Yad. Fiz, 27 67; (1978 Sov. J. Nucl, Phys. 27 351)

Gudima K K, Iwe H and Toneev V D 1979 J. Phys, G: Nuch Phys. 5229

Das Gupta S and Lam C S 1979 Phys. Rev. C 201192

Gustafsson H A et al 1984 Phys. Rev. Lett. 521590

Gutbrod H H, Poskanzer A M and Ritter H G 1989a Rep. Proc. Phys. 521267

Gutbrod H H, Kampert K H, Kolb B W, Poskanzer A M, Ritter H G and Schmidt H R 1989b Phys. Lett. 219B 2617

Gutbrod H H, Kampert K H, Kolb B W, Poskanzer A M, Ritter H G and Schmidt H R 1990a Z. Phys. A 33757

Gutbrod H H, Kampert K H, Kolb B W, Poskanzer A M, Ritter H G, Schicker R and Schmidt H R 1990b Phys. Rev. C 42640

Gyulassy M, Fraenckel K A and Stöcker H 1982 Phys. Lett. 110B 185

ter Haar B and Malfiet R 1986 Phys. Rev. Leth. 17210

ter Haar B and Malfliet R 1987 Phys. Rep. 149207

Hahn D and Stöcker H 1988 Nucl. Phys. A 476718

Halbert E C 1981 Phys. Rev. C 23295

Hartnack C 1992 PhD Thesis, University of Frankfurt, 1992 GSI-Report-93-05

Hjort $\mathrm{E}$ and the EOS/TPC collaboration 1994 Advances in Nuclear Dynamics Proc, 9th Winter Workshop on Nuclear Dynamics ed W Bauer and B Back, (Singapore: World Scientific) to be published

Hofmann H and Nix J R 1983 Phys. Lett. 122B 117

Horiuchi H 1991 Nucl. Phys. A $522257 \mathrm{c}$

Hubele J et al 1991 Z. Phys. A 340263

Hüfner J and Knoll J 1977 Nucl. Phys. A 290460

Iyanow Y B, Mishustin I N and Satarov L M 1985 Nucl. Phys. A 433713

Jacak B V, Westfall G D, Gelbke C K, Harwood L H, Lynch W G, Scott D K, Stöcker H, Tsang M B and Symons T L M 1983 Phys. Rev. Lett. 511846

Jacak B V et al 1987 Phys. Rev. C 351751

Jakobsson B, Jonsson G, Lindkvist B and Oskarsson A 1982 Z Phys. A 307293

Kampert K H 1989 J. Phys. G: Nucl. Phys. 15691

Keane D, Chu S Y, Fung S Y, Liu Y M, Qino L, J, VanDalen G, Vient M, Wang S, Molitoris J J and Stöcker $\mathbf{H}$ 1988 Phys. Rev. C 371447

Kim Y D et al 1991 Phys. Rev. Lett. 6714

Kim Y D, de Souza R T, Bowman D R, Carlin N, Gelbke C K, Gong W G, Lynch W G, Phair L, Tsang M B and Zhu F 1992a Phys. Rev. C 45338

Kim Y D, de Souza R T, Gelbke C K, Gong W G and Pratt S 1992b Phys. Rev. C 45387

Kuhnet Ch et al 1993 Phys. Rev. C 481232

Kiselev S M 1984 Yad. Fiz. 39 32; (1984 Sov. J. Nucl. Phys. 39 18)

Kiseley S M 1986 Yad. Fiz. 44 610; (1986 Sov. J. Nucl. Phys. 44 610)

Kiselev S M and Pokroskil Yu E 1983 Yad. Fiz, 38 46; (1983 Sov. J. Nucl. Phys. 38 46)

Kitazoe Y, Sano M, Yamamura Y, Furutani H and Yamamoto K 1984 Phys. Rev. C 29828

Koch V, Blattel B, Cassing W and Mosel U 1991 Nucl. Phys. A 532715

Konopka 1991 Diploma Thesis, University of Frankfurt, 1991 unpublished

Konopka J, Peilert G, Stöcker H and Greiner W 1993 Prog. Part. Nucl. Phys. 30301

Koonin S E 1975 PhD Thesis (MIT)

Koonin S E and Randrup J 1987 Nucl. Phys. A 474173

Kopylov G I 1974 Phys. Lett. 50B 472

Kramer P and Saraceno S 1981 Lecture Notes in Physics 140 (Berlin: Springer)

Krofcheck D et al 1989 Phys. Rev. Lett. 632028

Krofcheck D et al 1992 Phys. Rev. C 461416

Kruse H, Jacak B V and Stöcker H 1985a Phys. Rev. Lett. 54289

Kruse F, Jacak B V, Molitoris J J, Westfall G D and Stöcker H 1985b Phys. Rev. C 31770

Lagaris I E and Pandharipande V R 1981 Nucl. Phys. A 359349

Metropolis N, Metropolis A W, Rosenbluth M N, Teller A H and Teller E 1953 J. Chem. Phys. 211087

Mihustin I N, Russkikh V N and Satarov L M 1989 Nucl. Phys. A 494595

Minich R W, Agarval S, Bujak A, Chuang J, Finn J E, Gutay L J, Hirsch A S, Porile N T, Scharenberg R P and Stringfellow B C 1982 Phys. Lett. 118B 458

Molitoris J J and Stöcker H 1985 Phys. Lett. 162B 47

Molitoris J J, Hoffer J B, Kruse H and Stöcker H 1984 Phys. Rev. Lett. 53899

Molitoris J J, Stocker H and Winer B L 1987 Phys. Rev. C 36220 
Molitoris J J, Hahn D, Alonso C, Collazo I, Alessandris P D, McAbee T, Wilson J and Zingman Z 1988a Phys. Rev. C 371014

Molitoris J J, Bonasera A, Winer B L and Stöcker H 1988 b Phys. Rev. C 371020

Müller E 1990 J. Phys. G: Nucl. Phys. 161571

Mustafa M G, Blann M, Ignatyuk A V and Grimes S M 1992 Phys. Rev. C 451078

Myers W D 1978 Nucl. Phys. A 296177

Nagamiya S, Lamaire M C, Moeller E, Schnetzer S, Shapiro G, Steiner H and Tanibata I 1981 Phys. Rev. C 24 971

Nakai T and Takagi S 1984 Prog. Theor. Phys. 71 1005

Nix J R and Strottman D 1981 Phys. Rev. C 232548

Nordheim L W 1928 Proc. R. Sroc. A 49689

Ogilvie C A, Cebra D A, Clayton J, Howden S, Karn J, Vander Molen A, Westfall G D, Wilson W K and Winfield J S 1989 Phys. Rev. C 40654

Ogilvie C A et al 1990 Phys. Rev. C 43 R10

Ogilvie C A et al 1991 Phys. Rev. Lett. 671214

Ohtsuka N, Linden R, Faessler A and Malik F B 1987 Nucl. Phys. A 465550

Ono A, Horiuchi H, Maruyama T and Ohnishi A 1992 Prog. Theor. Phys, 871185

Panagioutou A D, Curtin M W, Toki H, Scott D K and Siemens P J 1984 Phys. Rev. Lett. 52496

Paul P Private Communication and Preprint State University of Stony Brook

Peilert G 1992 PhD Thesis, University of Frankfurt, 1992 GSI-Report-92-13.

Peilert G, Randrup J, Stöcker H and Greiner W 1991 Phys. Lett. 260B 271

Peilert G, Rosenhauer A, Aichelin J, Stöcker H and Greiner W 1988 Mod. Phys. Lett. A 3459

Peilert G, Sangster T C, Namboodiri M N and Britt H C 1993 LLNL Preprint UCRL-JC-115713 to be published

Peilert G, Stöcker H, Greiner W, Rosenhauer A, Bohnet A and Aichelin J 1989 Phys. Rev. C 391402

Peilert G, Stöcker H, Greiner W, Blann M and Mustafa M G 1992 Phys. Rev. C 461457

Pochodzalla J, Trautmann W and Lynen U 1989 Phys. Lett. 232B 41

Randrup R 1979 Nucl. Phys. A 314429

Randrup J and Koonin S E 1981 Nucl. Phys. A 356223

Rischke D, Schaffner J, Gorenstein M, Schäfer A, Stocker H and Greiner W 1992a Z. Phys. C 56325

Rischke D, Gorenstein M, Schäfer A, Stöcker H and Greiner W 1992b Phys. Lett. $278 B 19$

Ritter H G et al 1985 Nucl. Phys. A 447 3c

Rosenhauer A, Csernai L P, Maruhn J A and Greiner W 1984 Phys. Scr. 3045

Rosenhaver A, Maruhn J A, Greiner W and Csernai P 1987 Z. Phys. A 326213

Sandoval A et al 1980 Phys. Rev. C 211321

Sangster T C et al 1992 Phys. Rev. C 461404

Sangster T C, Ajitanand N N, Begemann-Blaich M, Blaich Th, Britt H C, Elmaani A and Namboodiri M N 1993 Prog. Part. Nucl. Phys. 30189

Satarov L M 1990 Sov, J. Nucl. Phys. 52264

Scheid W, Ligensa R and Greiner W 1968 Phys. Rev. Lett. 211479

Scheid W, Müller H and Greiner W 1974 Phys, Rev. Lett. 32741

Schlagel T J and Pandharipande V R 1987 Phys. Rev. C 36162

Schopper E 1937 Naturwiss. 46749

Schopper E and Schopper E M 1939 Phys. Z, 22

Schmidt H R 1991 Int. J. Mod. Phys. 63865

Schmidt H R and Schukraft J 1993 J. Phys. G: Nucl. Part. Phys. 191705

Schmidt W 1989 PhD Thesis, University Frankfurt 1989 GSI Report 89-16

Schürmann B 1988 Mod. Phys. Lett. A 121137

Scobel W, Traband M, Blann M, Pohl B A, Remington B A, Byrd R C, Foster C C, Bonetti R, Chiese C and Grimis S 1990 Phys. Rev. C 412010

Serot B and Walecka J D 1985 Advance in Nuclear Physics (New York: Plenum)

Serot B and Walecka J D 1986 Adv. Nucl. Phys. 161

Sharma M M 1989 Proc. NATO Advanced Study Institute on the Nuclear Equation of State, Peniscola, Spain (NATO ASI Series, Series B) vol 216A, ed W Greiner and H Stöcker p 661

Siemens P J 1983 Nature 305410

Skyrme T H R 1959 Nucl. Phys. 9615

Sobel M I, Siemens P J, Bondorf J P and Bethe H A 1975 Nucl. Phys. A 251502

Sorge H, Stöcker H and Greiner W 1989 Ann. Phys. 192266

Sorge H, von Keitz A, Matticllo R, Stöcker H and Greiner W 1991 a Z. Phys. C 47629

Sorge H, von Keitz A, Mattiello R, Stöcker H and Greiner W $1991 \mathrm{lb}$ Nucl. Phys. A $52595 \mathrm{c}$ 
Sorge H, Mattiello R, Stöcker H and Greiner W 1992a Phys. Rev, Lett. 68286

Sorge H, Berenguer M, Stöcker $H$ and Greiner W 1992b Phys. Lett. 289B 6

de Souza R T et al 1991 Preprint MSUCL-785

Stamer S 1992 PhD Thesis University Hamburg, 1992

Stevenson J D 1978 Phys. Rev. Lett. 411702

Stevenson J D 1980 Phys. Rev. Lett. 451773

Stock R 1975 Nucl. Phys. A. 237365

Stock R et al 1980 Phys. Rev. Lett. 441243

Stöcker H, Maruhn J A and Greiner W 1979 Z. Phys. A 290297

Stöcker H, Maruhn J A and Greiner W 1980a Phys. Rev. Lett. 44725

Stöcker H, Cusson R Y, Maruhn J A and Greiner W 1980b Z. Phys. A 294125

Stöcker $\mathrm{H}$ et al 1981 Phys. Rev. Lett. 471807

Stöcker H, Csernai L. P, Graebner G, Buchwald G, Kruse H, Cusson R Y, Maruhn J A and Greiner W 1982 Phys. Rev. C 251873

Stöcker H, Buchwald G, Gräbner G, Subramanian P, Maruhn J A, Greiner W, Jacak B V and Westfall G D 1983 Nucl. Phys. A $40063 \mathrm{c}$

Stöcker H and Greiner W 1986 Phys. Rep. 137

Sultivan J P et al 1990 Phys. Lett. 249B 8

Theis J, Gräbner G, Buchwaldt G, Maruhn J A, Greiner W, Stöcker H and Polonyi J 1983 Phys. Rev. D 282286

Trabandt M, Scobel W, Blann M, Pohl B A, Byrd R C, Foster C C, Grimes S M and Bonetti R 1989 Phys. Rev. C 39452

Trockel R et al 1987 Phys. Rev. Lett. 592844

Trockel R et al 1988 Phys. Rev. C 38576

Trockel R et al 1989 Phys. Rev. C 39729

Tsang M B et al 1993a Phys. Rev, Lett. 711502

Tsang M B et al 1993b Phys. Rev. C at press

Uehling E A and Uhlenbeck G E 1933 Phys. Rev, 43552

Valta P 1991 Diploma Thesis, University of Heidelberg, $199 \mathrm{I}$ unpublished

Valta $P$ et al 1992 Nucl. Phys. A $518417 \mathrm{c}$

Vautherin D and Brink D M 1972 Phys. Rev. C 5626

Waddington C J and Freier P S 1985 Phys. Rev, C 31888

Waldhauser B M, Theis J, Maruhn J A, Stöcker H and Greiner W 1987 Phys, Rev. C 361019

Waldhauser B M, Theis J, Maruhn J A, Stöcker H and Greiner W 1988 Phys. Rev. C 381003

Walecka J D 1974 Ann. Phys. 83547

Warwick A l et al 1983 Phys. Rev. C 271083

Weisskopf V and Ewing D H 1940 Phys. Rev. 57472

Westfall G D, Gosset J, Johansen P J, Poskanzer A M, Meyer W G, Gutbrod H H, Sandoval A S and Stock R 1976 Phys. Rev. Lett. 271202

Westfall G D et al 1993 Phys. Rev. Lett. 711986

Wilets L, Henley E M, Kraft M and MacKellar A D 1977 Nucl. Phys. A 282341

Wilets L, Yariv Y and Chestnut R 1978 Nucl. Phys. A 301359

Wilson W K et al 1990 Phys. Rev. C 411881

Yariv Y and Fraenkel Z 1979 Phys. Rev. C 202227

Yariv $Y$ and Fraenkel $Z 1981$ Phys. Rev. C 24488

Zajc W et al 1984 Phys. Rev. C 292173 